\title{
The Synthesis of Alginate Microparticles and Nanoparticles
}

\section{Yuri S Pestovsky* and Agustino Martínez-Antonio}

Center for Research and Advanced Studies of the National Polytechnic Institute (Cinvestav), Genetic Engineering Department, Mexico

*Corresponding author: Yuri S Pestovsky, Center for Research and Advanced Studies of the National Polytechnic Institute

(Cinvestav), Genetic Engineering Department, Mexico

\begin{abstract}
Alginate is a natural polysaccharide that is widely used as a component of pharmaceuticals and in food industry. Alginate particles can be used for encapsulation of substances with the necessity of prolonged release. They can also provide appropriate microenvironment for cells. Here the methods of the synthesis of alginate beads, micro- and nanoparticles are reviewed with special attention to the calcium alginate ones. The results from publications that did not deal with alginate particles but, to our opinion, could be applied in this field are also included in order to give an outline for possible future research. The suggested applications of the particles are mentioned as well. The two main methods for the synthesis of calcium alginate particles are internal and external gelation, but the external gelation techniques can be themselves subdivided into several subtypes. Currently, a technique being able to produce alginate nanoparticles with any desirable size does not exist. We analyze the possibilities of employing aerosolization method for this purpose. The potentials to overcome the problem of burst release of the encapsulated substances by means of cyclodextrin inclusion complexes and employing additional crosslinking agents are also discussed. The clinical application of alginate nanoparticles is still limited because of the burst release of encapsulated drugs and the poor size control of the particles formed. Further research must concentrate on overcoming these problems and on topical application of alginate particles without entering bloodstream rather than on investigation of model drug release in vitro without taking the above-mentioned problems into account.
\end{abstract}

Keywords: Calcium alginate; Alginate beads; Alginate microparticles; Alginate nanoparticles; Drug encapsulation; Particle size control; Aerosol

\section{Introduction}

Alginates are polysaccharides. The commercially available ones come from brown algae. They are linear copolymers of $(1 \rightarrow 4)$-linked units of $\beta$-D-mannuronic acid and $\alpha$-L-guluronic acid. The molar ratio between them and their distribution along the commercial polymer depend on the algal source, its location, age, collection season and extraction technique. Guluronic acid residues can form so-called egg-box complexes with calcium ions or some other divalent metal cations leading to gel formation (Figure
1). The name 'egg-box' is used because, if depicted schematically, the cations look like eggs situated inside puckered boxes formed by four guluronic acid residues of two superimposed chains. Mannuronic acid residues have much less affinity to metal ions [1]. Barium ions have more affinity to alginate than the calcium ones. If reacted with calcium alginate at lower concentrations, they create new gelling junctions. At higher concentrations, barium ions also displace calcium ions from existing junctions [2].

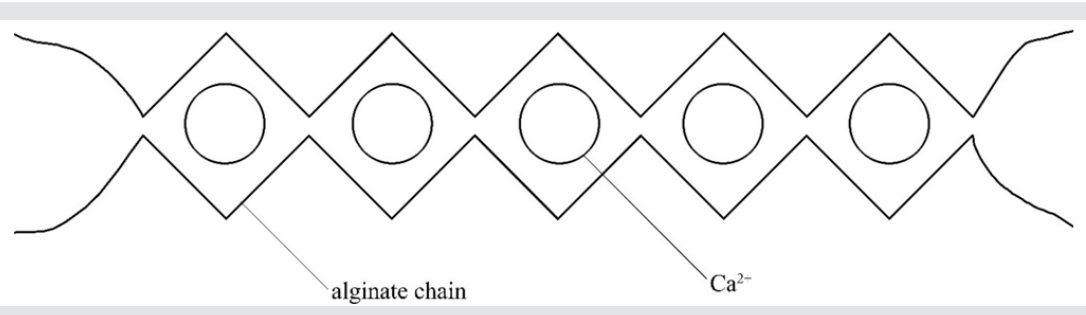

Figure 1: The egg-box model. 
To extract alginate, algae are usually washed with organic solvents and water, dried and milled. After acid pretreatment, alginate is solubilized with $\mathrm{Na}_{2} \mathrm{CO}_{3}$. The crude extract is concentrated, dialyzed against water and then freeze-dried or precipitated with ethanol. Acidification or treatment with $\mathrm{Ca}^{2+}$ can be used instead. Brown algae are abundant in nature; however, the possibility of cultivating them exists as well [1].

The viscosity of aqueous sodium alginate solution rapidly increases with its concentration. For example, the addition of $10 \%$ of alginate to water leads to a $\sim 100$-fold increase in viscosity. But the poly electrolyte nature of alginate has little effect on its hydration, and in the above example less than $3.7 \%$ of the water molecules present in solution is involved in alginate hydration. Such a large viscosity increase is determined by the polysaccharide network, with large bulk-like water pools present between the polysaccharide chains [3].

Sodium alginate may act as a mucoadhesive polymer. A comparative study of adhesion between buccoadhesive compacts and pig buccal mucosa or sodium alginate solution revealed that the results were of similar performance [4]. Sodium alginate was proposed as a mucoadhesive component of a nasal gel [5] or in buccal patches containing salbutamol sulfate [6]. Sodium alginate conferred in situ gelling mucoadhesive properties and retarded drug release from liquid rectal suppositories. These suppositories were successfully tested on Guinea pigs to alleviate symptoms of histamine-induced bronchospasm [7]. Sodium alginate was also evaluated as an excipient in salbutamol sulfate sublingual films [8] and tablets [9]. However, drug release was found to be too slow in the films [8] or too rapid in tablets [9]. Only salbutamol sulfate tablets formulated from granules containing mastic and sodium alginate excelled commercial tablets in the terms of drug release when tested on rabbits [10]. In combination with hydroxy propyl methyl cellulose and propylene glycol sodium alginate was used in the formulation of terbutaline sulfate sublingual films [11]. Sodium alginate could be also used as a component of plugs for water-soluble parts of crosslinked gelatin capsules containing pellets with encapsulated salbutamol sulfate. The plug absorbed the surrounding fluid, and began to release the drug through the swollen matrix and was finally ejected out of the capsule by erosion of the material. The usability of the system was shown on rabbits [12].

Chitosan-alginate complex was proposed as an excipient for orodispersible tablets, and their disintegration time was so short that it was even referred to as a 'super dis-integrant' [13]. One optimized formulation containing the excipient for 5-fluorouracil tablets, suitable for trans buccal and rectal drug delivery, contained this chitosan-alginate complex along with the same components un-complexed in order to avoid burst release and to improve the mucoadhesive properties [14].

Sodium alginate itself also has a therapeutic effect. When admixed to foods for diabetic human patients, it decreased gastric emptying rate and rises glucose in blood, serum insulin and plasma C-peptide levels [15]. Orally administered, sodium alginate significantly alleviated small intestinal enteritis in rats, caused by treatment with the anti-inflammatory drug indomethacin, and this relief seemed to be independent of the sodium alginate viscosity administered [16]. Oral disposal of sodium alginate to rats with colitis led to a significant reduction of colonic damage, decreased lesion formation $[17,18]$ and inhibited mucosal injury [17].

Orally administered alginate oligosaccharide obtained from hydrolysis of sodium alginate by Bacillus subtilis improved histopathological and biochemical parameters of mice having ovalbumin-induced asthma in a dose-dependent manner [19]. Rats fed with sodium alginate drank more water, and their urine volume and $\mathrm{pH}$ rose sharply. In contrast, calcium alginate caused very little changes in the same parameters [20].

Commercially available calcium alginate swabs were used for sampling nasal flora for subsequent DNA extraction [21]. The mucoid exopolysaccharide produced by the pathogenic bacterium Pseudomonas aeruginosa is alginate, but it has low immunogenicity if it is not conjugated with a carrier protein. Even in the form of conjugate it is non-toxic if administered intraperitoneally to mice or guinea pigs and non-pyrogenic if administered intravenously to rabbits [22]. When alginate beads with encapsulated tumor cells were implanted to mice, a process of angiogenesis was observed in the implants zone. The beads were prepared from commercially available alginate [23]. It may be considered as a further proof of alginate biocompatibility.

Sodium alginate was also shown to be beneficial for agriculture. Being administered as dietary supplement to the white shrimp Litopenaeus vannamei, it acted as an immunostimulant and improved its resistance against the attack of Vibrio alginolyticus bacterium [24]. Sodium alginate digested with alginate lyase promoted root elongation of rice, carrot [25], lettuce [26] and barley plants $[27,28]$ even under hypoxic conditions [28]. It was hypothesized that digested alginate might initiate some signal transduction pathway $[27,28]$. Under hypoxic conditions digested alginate also caused enhancement of the activity of the enzymes regenerating $\mathrm{NAD}^{+}[28]$.

Alginate oligomers promoted the germination of unhulled rice and Komatsuna seeds as well as tobacco callus differentiation. The mixture of oligomers was assumed to contain so-called oligosaccharine, an oligosaccharide inducing unusual proliferation and/or differentiation of plant cells. There are several kinds of oligosaccharines. They act as a chemical signal for the stimulation of hormone synthesis [29]. The promoting effect of alginate oligosaccharides on root formation and growth in rice was mediated by endogenous indole-3-acetic acid [30].

But proliferation of the microalga Chlamidononas reinhardtii was repressed by the same oligomers [29]. It should be noted that only digested sodium alginate shows this effect. And the possibility of alginate degradation by the lyases of soil bacteria is assumed [28]. Therefore, it is speculated that these active substances can be formed from alginate under natural conditions.

Another notable advantage of alginate is its ability to bind micronutrients. Some important $\mathrm{Mn}, \mathrm{Cu}, \mathrm{Zn}$ and Mo fertilizers are $\mathrm{MnCl}_{2}, \mathrm{CuSO}_{4} \cdot 5 \mathrm{H}_{2} \mathrm{O}, \mathrm{ZnCO}_{3}$ and $\mathrm{Na}_{2} \mathrm{MoO}_{4} \cdot 2 \mathrm{H}_{2} \mathrm{O}$, respectively [31]. 
Manganese can be complexed with alginate by addition of $\mathrm{MnCl}_{2}$ to the gelling solution of $\mathrm{CaCl}_{2}$ or $\mathrm{BaCl}_{2}$, and slow release of manganese ions from the beads into physiological saline has been reported [32] because the affinity of alginate to $\mathrm{Ca}^{2+}$ is higher than to $\mathrm{Mn}^{2+}$ [33]. The beads containing $\mathrm{Ba}$ and $\mathrm{Mn}$ could be used for manganeseenhanced magnetic resonance imaging and were tested on rats [32]. The use of $\mathrm{CuSO}_{4}$ [34] or $\mathrm{CuCl}_{2}$ [35] as a gelling solution led to copper alginate hydrogel being able to release copper ions into simulated body fluid [34] or into phosphate buffer (pH 6) [35] in a prolonged manner. The use of basic zinc carbonate for zinc alginate hydrogel formation using internal gelation method has also been reported. The hydrogel was active against $E$. coli [36]. Molybdenum can be adsorbed by preformed calcium alginate beads preferably in the form of $\mathrm{H}_{2}\left[\mathrm{MoO}_{4}\right]$ or $\left[\mathrm{Mo}\left(\mathrm{H}_{2} \mathrm{O}\right)_{6}\right]^{3+}$ at $\mathrm{pH} 2$ and released back up to $50 \%$ into $0.1 \mathrm{M} \mathrm{HCl}$. If radioactive molybdenum is used, the method is suitable for radiotherapy [37]. Copper ions from $\mathrm{CuCl}_{2}$ may also be adsorbed onto preformed calcium alginate beads [38]. Copper alginate shows activity against Staphylococcus aureus, Staphylococcus epidermidis, Staphylococcus pyogenes and E. coli [34].

The biodegradability of unmodified alginate particles, their ability to bind micronutrients and the beneficial effect of alginate make them promising carriers of agrochemicals. This is especially important because in a recent review [39] the authors expressed great concerns regarding the use of nanoparticles in agriculture because of the negative impact of metal and oxide nanoparticles on soil microorganisms, earthworms and even on cultivated plants.

Alginate beads can also have industrial applications:

- Wastewater treatment. The beads with encapsulated horseradish peroxidase could be reused up to 3 times, although the encapsulation decreased enzyme activity in comparison with the free enzyme [40]. In another report, the efficiency of phenol removal by encapsulated horseradish peroxidase was demonstrated by reducing to half the initial phenol quantity after only 5 reaction cycles [41].

- $\quad$ Food industry. Xylanase immobilized in alginate beads may be used for fruit juice clarification [42]. Lactobacillus helveticus and Streptococcus thermophilus immobilized in alginate beads were intended for use as lactic starters in milk fermentation [43]. Alginate particles with encapsulated healthy nutrients can also be used as components of functional foods.

- Enzyme production by cells immobilized in alginate beads. A good example is glucoamylase [44]. In this case fungus Thermomucor indicae-seudaticae was immobilized in alginate beads, and cane molasses was used as a cheap medium [44].

In all these applications alginate is exploited because of its natural origin, i.e. it cannot be a harmful admixture if separated incompletely. Beads (and not nanoparticles) are chosen because they can be easily separated by sedimentation. As could be seen above, not only enzymes themselves but also enzyme-producing bacteria can be immobilized in alginate beads.
In contrast to metal nanoparticles, alginate particles can be modified either before or after their synthesis. In the former case, bulk alginate is modified and then used to prepare the particles. This way is usually preferable from the two options because it avoids the leak or destruction of encapsulated substance during the modification. The chemical modification of alginate is reviewed widely in [45].

Alginate microparticles and nanoparticles are usually used to encapsulate and carry various substances, and the goal of many studies is to achieve sustained release of them. It should be noted, however, that the results of experiments dealing with the release of poorly water-soluble drugs might often be misinterpreted, because the drug not found in the solution is assumed to remain encapsulated in the particles. However, it may decompose after the release or simply precipitate out. Supersaturated solutions with varying extent of supersaturation can also be formed, making the results irreproducible. Special care must be taken in the case of putting the particles into a dialysis bag, because the films act as an additional diffusion barrier. If centrifugation is used for separation of the medium with the released substance from the nanoparticles, the pressure generated during the process can disturb the equilibrium. It can also make difficult resuspending the nanoparticles in the fresh portion of medium for further incubation [46].

For consistency, throughout this review we will use the following terms (even if different names for them were used in the respective publications):

- Encapsulation efficiency: the percentage of the substance that was encapsulated (i.e. not lost).

- Loading efficiency (expressed in percent): the ratio of the weight of the successfully encapsulated substance regarding to the total weight of the particle. Some authors calculate loading efficiency using different formulae, but we will give their values without a special discussion. We have rounded encapsulation and loading efficiencies as well as zeta potentials to the nearest integer values.

- Nanoparticles are considered smaller than $1 \mu \mathrm{m}$. Microparticles have size from 1 to $1000 \mu \mathrm{m}$. Beads have size in a millimeter range. We have rounded bead size to the first decimal place.

Also, some authors term their particles 'microcapsules'. However, we will use this term only if they have demonstrated or at least assumed the presence of a liquid core in their particles. In other cases, we will refer them to as microparticles.

\section{Alginate-chitosan particles}

Since alginate is a polyanion and chitosan is a polycation, they can form a polyelectrolyte complex upon mixing, provided both of them are charged, i.e. at suitable $\mathrm{pH}$. This mixture can spontaneously form particles. The $\mathrm{pK}_{\mathrm{a}}$ of alginate carboxyl group is close to 5 , and that of the ammonium group of chitosan is about $6.2[1]$. 
Alginate-chitosan nanoparticles were prepared by dropwise addition of a chitosan solution containing glutathione into an alginate solution at $\mathrm{pH} \mathrm{4}$, under stirring. If prepared at 0.75 alginate: chitosan ratio, the formed nanoparticles with encapsulated glutathione had the following characteristics: size $361 \mathrm{~nm}$, polydispersity index 0.33 , zeta potential $27 \mathrm{mV}[47,48]$. At 1.5 alginate: chitosan ratio, the values were $212 \mathrm{~nm}, 0.4$ and $23 \mathrm{mV}$, respectively, although the storage stability decreased, making these nanoparticles less suitable for application. In the same way, the $\mathrm{pH}$ increase from 5.0 to 6.5 and further caused aggregation [48]. The encapsulation efficiency was $27 \%$ [47] or $80 \%$ at ratio 0.75 and fell to $1 \%$ at ratio 1.5 [48]. The respective investigations were aimed to achieve the synthesis of mucoadhesive nanoparticles with an encapsulated NO donor needed for treat important diseases because of the multifaceted role of NO in vivo. Therefore, encapsulated glutathione was nitrosated inside the nanoparticles by adding sodium nitrite to the solution. S-nitrosoglutathione decomposition at $400 \mu \mathrm{M}$ was delayed by its encapsulation in the nanoparticles. At $18 \mu \mathrm{M}$ encapsulated S-nitroso glutathione was not cytotoxic to cultured Chinese hamster lung fibroblast cells (V79), whereas free S-nitroso glutathione was slightly cytotoxic at the same concentration. This assay could enable the use of these anti microbial nanoparticles in pharmaceutical applications such as wound healing without severe side effects $[47,48]$.

Later, the same technique was used by the same group to encapsulate mercaptosuccinic acid and nitrosate it inside the particles. In this case, the hydrodynamic size of the nanoparticles was $\sim 750 \mathrm{~nm}$. The encapsulation efficiency was $89 \%$. Burst release of NO in aqueous solution was followed for 4 hours, although the release in the normal mode continued for 6 hours more. These nanoparticles were assayed for topical application for bovine mastitis. The minimal inhibitory concentration of the nanoparticles for Staphylococcus aureus determined in vitro was $125-250 \mu \mathrm{g} / \mathrm{ml}$. The number of colony forming units was 10-fold and 1000-fold lower after bacteria were incubated with nitrosated nanoparticles at $500 \mu \mathrm{g} / \mathrm{ml}$ for 4 and 7 hours, respectively, compared with bacteria growth in the presence of empty nanoparticles at the same concentration and time. The CFU drastically decreased further upon the addition of a second dose of nitrosated nanoparticles. For E. coli the minimal inhibitory concentration exceeded $2000 \mu \mathrm{g} / \mathrm{ml}$, i.e. these nanoparticles were inefficient against this bacterium.

The $50 \%$ cytotoxicity concentration of the nanoparticles for cultured HEp-2 cells was $640 \mu \mathrm{g} / \mathrm{ml}$. Chitosan nanoparticles without alginate at the same concentrations of the acid released more NO at higher rates. Nevertheless, it was concluded that NO-releasing nanoparticles might be used to combat bacteria for treating and preventing bovine mastitis [49].

Spherical alginate-chitosan beads with encapsulated lemongrass oil having size of $1.8-2.1 \mathrm{~mm}$ displayed significant antibacterial and antioxidant activity. For unencapsulated oil the same activity was observed only at higher concentration. This beneficial action was attributed to the strong interaction between chitosan and the oil. This kind of beads has potential applications as a greener agent for medical purposes [50].
The following advantages of alginate-chitosan particles can be underlined:

- $\quad$ Chitosan can enhance drug bioavailability by its capacity of infiltration into the mucus layer of the small intestine with subsequent opening of tight junctions of epithelial cells [51].

- Unlike calcium alginate, alginate-chitosan polyelectrolyte complex cannot be disintegrated by chelatoring agents.

Their main disadvantage could be the necessity to use an acidic solution of chitosan because of its insolubility at neutral $\mathrm{pH}$.

\section{Preparation of alginate particles without employing gelation}

Now we describe the techniques for preparation of particles from bulk sodium alginate or its solution as well as spontaneous formation of particles of modified alginic acid in water. The resultant particles are usually intended to be ready to use. However, dry sodium alginate particles can be later treated with $\mathrm{CaCl}_{2}$ solution in order to convert them to calcium alginate particles.

There exists a patented technique for producing alginate, cellulose, starch or collagen particles from bulk substances by ball milling with the possibility to control particle size from $100 \mathrm{~nm}$ to $50 \mu \mathrm{m}$. The resultant nanoparticles containing therapeutic proteins have shown efficacy in treating solid tumors, single dose vaccination, and oral delivery. For instance, tumor-bearing mice that received these nanoparticles containing Texas red and cisplatin showed significant tumor size diminishing. If the same nanoparticles were coupled to dendritic cell-binding peptide and contained encapsulated pneumococcal surface protein A, together with an adjuvant, they were effective to combat the bacterial load of the mice that was reduced (in the terms of infected tissue volume) after exposition to nanoparticles. The nanoparticles produced by the same milling technique were also used to induce passive immunity against anthrax toxin in mice by means of oral delivery of monoclonal antibodies developed versus anthrax toxin [52].

Another technique consists in dropwise addition of pure ethanol or acetone to $1 \%$ sodium alginate in water containing drug solution in dimethyl formamide. Mixing [53] and cooling down to $3-5{ }^{\circ} \mathrm{C}$ is needed during the process. At low mixing speed aggregation was observed [54]. These microparticles can be separated by filtration, washed with the same solvent and dried on air, in a heating oven [53] or in a desiccator [54]. Using nitrofurazone as an example of an encapsulated drug, the loading efficiency and yield of microparticles decreased as the particle size increased from 5 to $30 \mu \mathrm{m}$ with ethanol dripping rate increasing. The presence of $0.1 \%$ ammonia [54] (pH 8-9) and of a surfactant was needed in order to avoid particle aggregation in the case of nitrofurazone. Other drugs, viz. acridone, tetracycline, dibazole and metronidazole were encapsulated in the same way (but without ammonia), although the encapsulation conditions needed to be optimized for every drug separately. The yield varied from $31 \%$ for metronidazole to $77.5 \%$ for tetracycline, and the loading efficiency varied from $2 \%$ for metronidazole to $43 \%$ for nitrofurazone [53]. In a later publication, the same group reported that in the case of nitrofurazone the 
yield of microparticles was $81 \%$ with a loading efficiency of $34 \%$. Spray drying instead of filtration was recommended to increase the yield [54]. The stability of nitrofurazone-loaded microparticles resuspended in water was reported to increase with $\mathrm{pH}$ [53]. At $1 \%$ and $2 \%$ of particles (nitrofurazone concentration was $0.34 \%$ and $0.68 \%$, respectively) these solutions were more active against $E$. coli, P. aeruginosa, P. vulgaris, $S$. aureus and B. subtilis than aqueous nitrofurazone solution having drug concentration less than $0.02 \%$ because of its insolubility. In the case of Candida albicans the same solutions of microparticles excelled in antifungal activity nitrofurazone solutions in DMSO with the concentrations of $1 \%$ and $2 \%$ [54]. The ability of the particles to form stable suspensions and to enhance drug solubility in water broadens the field of drug application $[53,54]$. The encapsulated drugs are expected to be more stable under ambient conditions [53].

Spray drying the sodium alginate solution containing the payload (caffeine-loaded peptidic nanoparticles) yielded microparticles having size of about $4 \mu \mathrm{m}$. The crosslinking with $\mathrm{CaCl}_{2}$ solution increased their mean size to $7.4 \mu \mathrm{m}$ but decreased their shrinkage and slowed down the release of caffeine into simulated gastric fluids. The particles are potentially bioactive because of the presence of antioxidant peptides [55]. Spray drying the solution containing sodium alginate, pectin and gentamicin sulfate at inlet temperature of $90{ }^{\circ} \mathrm{C}$ was used for wound dressing preparation. The volume diameter at the $50^{\text {th }}$ percentile (spanning from 310 to $1003 \mathrm{~nm}$ for various samples), the width of particle size distribution, water content and drug release rate increased with nozzle spray mesh diameter and with feed solution concentration at constant ratio of the components. Flowability of the powders, the adhesive strength of the gel formed from them in contact with simulated wound fluid as well as its activity at $0.25 \mathrm{mg} / \mathrm{ml}$ of gentamicin sulfate against Staphylococcus aureus and Pseudomonas aeruginosa showed the opposite tendency. Antimicrobial activity was expressed as the diameter of the zones of clearance around the two samples spotted on agar plates with the spread bacterial culture after incubation for 24 hours. For Staphylococcus aureus the activity of two samples was also tested in culture medium after 3, 6, 9 and 12 days of incubation. The particles were mainly spherical, but other shapes appeared when either feed concentration or mesh nozzle increased, and further increase led to large collapsed particles. All the particles were composed of smaller aggregated particles. The encapsulated drug was being released in simulated wound fluid in Franz-type diffusion cells for up to 5 days. Loading efficiency was around $24-27 \%$ with an encapsulation efficiency between 70 and $83 \%$, and for all the samples initial burst release was observed. At $40{ }^{\circ} \mathrm{C}$ and $75 \%$ relative humidity drug content was preserved for 6 months with only slight increase in water content. Swelling rate in contact with simulated wound fluid depended on particle size. The yield increased with feed solution concentration but decreased with nozzle spray mesh diameter. The nanoparticulate powder may be used as a self-consistent formulation having great potential application in the treatment of both acute and chronic infected wounds [56].

Low molecular weight alginic acid prepared by acid hydrolysis of sodium alginate formed nanoparticles itself (without calcium ions) when its hydroxyl groups were functionalized with oleoyl residues. The nanoparticles were loaded with vitamin $\mathrm{D}_{3}$ by addition of its solution to the reconstituted solution of vacuumdried nanoparticles. Loading efficiency increased with the vitamin concentration from 0.3 to $0.9 \%$, but the encapsulation efficiency also decreased from $68 \%$ to $46 \%$. Mean nanoparticle hydrodynamic diameter also decreased from $559 \mathrm{~nm}$ to $305 \mathrm{~nm}$, and particle formation rate was sped up when substitution degree increased. An unimodal particle size distribution was revealed. In simulated gastric fluid they retained spherical shape and released $\sim 40 \%$ of the encapsulated vitamin for 3 hours. But in simulated intestinal fluid they became irregularly shaped, their hydrodynamic diameter was $757 \mathrm{~nm}$ and burst release of $40 \%$ of the vitamin occurred, with $60 \%$ of the vitamin released after 7 hours. The nanoparticles can be used as oral carriers for liposoluble nutraceuticals [57].

The great disadvantage of sodium alginate particles is the very limited possibility of prolonged drug release because of sodium alginate solubility leading to fast disintegration of the particles. The particles of modified alginic acid offer convenient manipulation, but the need of its prior chemical functionalization limits the applicability of the technique for non-specialized, e.g. biomedical laboratories.

\section{Production of Alginate Particles Using Other Particles as Cores}

In this method, sodium alginate is physically adsorbed or covalently linked to the surface of other particles. The particles can also be formed already capped with alginate. In some cases subsequent gelation with $\mathrm{CaCl}_{2}$ is carried out. For $\mathrm{CaCO}_{3}$ particles there is a possibility of their generation simultaneously with alginate gelation.

Dropwise addition of chitosan nanoparticles with encapsulated bovine serum albumin modified with rhodamine isothiocyanate to sodium alginate solution at controlled $\mathrm{pH}$ yielded negatively charged nanoparticles having hydrodynamic diameter of several hundred nanometers depending on the solution composition. The highest diameters were registered in water. The nanoparticles successfully delivered the protein into cultured cells, with the localization depending on cell type. Significant increase in peroxide production by HCEC cells was observed at 300 and $600 \mu \mathrm{g} / \mathrm{ml}$ of empty nanoparticles after exposure for 4 hours. However, there was almost no superoxide production after either 4 or 24 hours of exposure. The metabolic activity of LN229 and MCF-7 cells remained unchanged for up to 72 hours of incubation with the empty nanoparticles. But MDA-MB-231 and HCEC cells displayed significantly decreased metabolic activity at nanoparticle concentration above $180 \mu \mathrm{g} / \mathrm{ml}$ after 72 hours of exposure, but not after 24 hours. Similar survival decrease at these concentrations of nanoparticles was observed for A549 cells. Dose dependencies acquired after 24 or 72 hours of exposure were almost the same. Survival of HT29 and CaCo2 cells was significantly increased only after exposure to $600 \mu \mathrm{g} / \mathrm{ml}$ of the nanoparticles for 72 hours. The nanoparticles have potential applicability as nanocarriers in cancer therapy [58]. A similar technique was used for enoxaparin encapsulation. The proposal was 
to evaluate nanoparticles loaded with this low molecular weight heparin for its oral delivery, controlled and prolonged release in order to improve patient compliance. In this, chitosan nanoparticles were covered with sodium alginate (applied in phosphate buffer) and treated with $\mathrm{CaCl}_{2}$. Parameters of the optimized formulation were as follows: average size $335 \mathrm{~nm}$, spherical, polydispersity index 0.37 , zeta potential $-31 \mathrm{mV}$, encapsulation efficiency $>70 \%$, drug release in simulated gastric fluid for 2 hours $2 \%$, in simulated intestinal fluid for 14 hours $\sim 60 \%$. Degradation and erosion of nanoparticles was identified as a possible drug release mechanism. The pharmacokinetic parameters of the drug given orally to fasted rats through cannula in a dose of $50 \mathrm{mg} / \mathrm{kg}$ body weight were improved. Nevertheless, those of intravenously administered free enoxaparin at $1 \mathrm{mg} / \mathrm{kg}$ were better. $75 \%$ of the encapsulated drug applied at $2 \mathrm{mg} / \mathrm{ml}$ reached across the intestine to the serosal fluid for 90 minutes, as shown in vitro by means of everted intestinal sac model. 900 IU of orally administered encapsulated drug reduced thrombus formation by $59 \%$ compared with buffer. Significant uptake of the nanoparticles by the intestinal mucosa for 1 hour was shown by administration of nanoparticles loaded with fluorescein isothiocyanate instead of the drug through gastric cannula to fasted rats. Therefore, the nanoparticles proved their utility as oral delivery vehicle for enoxaparin. Such a vehicle is a foremost requirement for non-invasive and non-hospitalized treatment of vascular disorders (deep vein thrombosis, pulmonary embolism and venous thromboembolism). But subcutaneously injected free drug was even better [51].

Microspheres loaded with dermatan sulfate were formed by its complexation with chitosan with subsequent addition of the resulting solution to sodium alginate solution followed by spray-drying. The spray-dried microspheres were collected in $\mathrm{CaCl}_{2}$ solution in $90 \%$ ethanol and then vacuum-dried. Their properties (including shape) significantly depended on buffer, $\mathrm{pH}$ and polyelectrolyte ratio, because these parameters influenced polyelectrolyte complex formation. The objective of the study was to achieve sustained delivery of dermatan sulfate from microspheres intended for its site-specific delivery. The particles had size around $2.3 \mu \mathrm{m}$ and zeta potential from -27 to $-48 \mathrm{mV}$.
Encapsulation efficiency was up to $96 \%$ with loading efficiency of around $4 \%$. They released dermatan sulfate in Tris buffer for more than 10 days. After incubation for 48 hours at $10 \mathrm{mg} / \mathrm{ml}$ in culture medium they increased growth of cultured mouse fibroblasts (NIH 3T3) up to $170-200 \%$. Free dermatan sulfate at $400 \mu \mathrm{g} / \mathrm{ml}$ had the same effect. Therefore, released dermatan sulfate was structurally intact and retained its bioactivity. The microspheres synthesized at pH 5 had slightly higher stimulatory effect than those synthesized at $\mathrm{pH}$ 3.5. A clear dose dependence of the stimulatory effect was observed at $1-10 \mathrm{mg} / \mathrm{ml}$ of the microparticles. If chitosan was omitted, the microparticles exhibited rapid burst release, and partially dissolved microspheres fused and formed a network. The microspheres have great potential to be used in tissue engineering applications because dermatan sulfate is an emerging therapeutic agent for tissue regeneration [59].

Another unusual technique was used for the encapsulation of fungicide prochloraz. To the oil-in-water emulsion of prochloraz stabilized by surfactant cetyltrimethylammonium bromide prehydrolized tetraethyl orthosilicate was added. It resulted in its further hydrolysis and silica shell formation around the pesticidecontaining drops. After a drying step the silica shell was further modified with amino groups and coupled with alginate using carbodiimide chemistry. The resulting microcapsules were dried again and treated with metal chlorides in solution. This was done in order to use them in agriculture as a nanofertilizer and nanopesticide at the same time. Their size (after drying and redissolving) was $\sim 2.5 \mu \mathrm{m}$ with homogenous distribution. Fungicide loading efficiency was about $30 \%(\mathrm{w} / \mathrm{w})$. Prochloraz release for 60 days in $30 \%(\mathrm{v} / \mathrm{v})$ methanol in water was shown. The degradation rate of free prochloraz increased with temperature and was also high under strong acidic and alkaline conditions. In contrast to this, the microcapsules were insensitive to $\mathrm{pH}$, protected the encapsulated fungicide from decomposition under ultraviolet irradiation, and temperature increase just caused some release alteration. They are expected to increase disease resistance of plants. The delivery system may be extended to other photosensitive or $\mathrm{pH}$-sensitive pesticides [60].

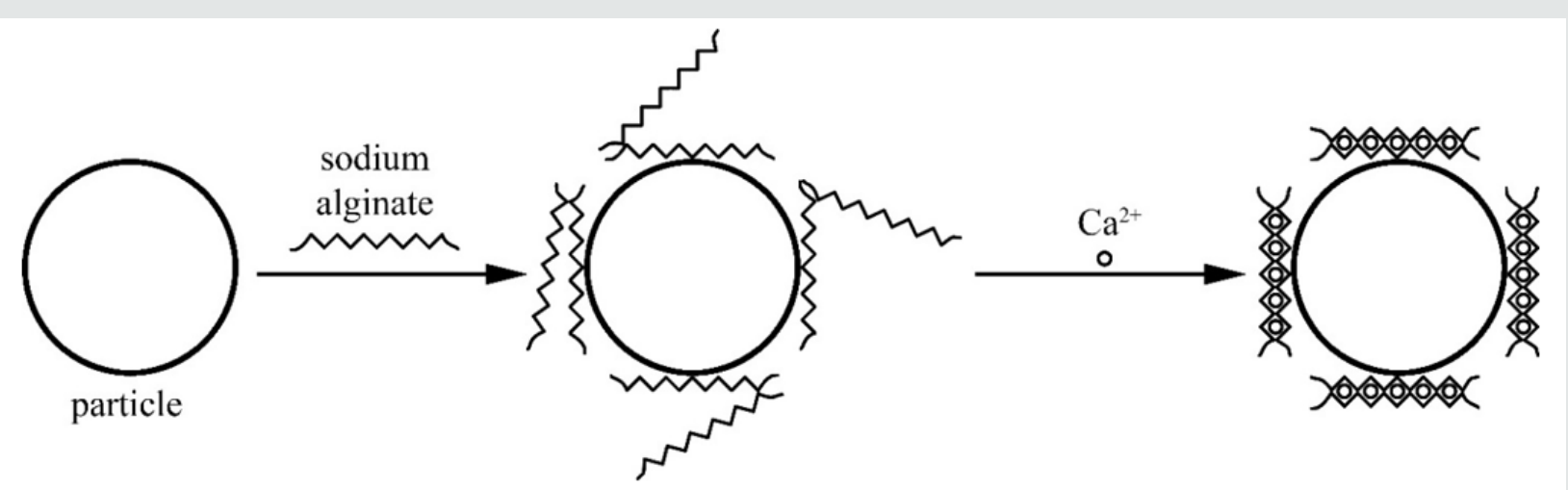

Figure 2: Production of alginate nanoparticles using other nanoparticles as cores.

Treatment of Triticum aestivum L. seeds with aqueous solution of patented silver nanoparticles capped with sodium alginate for 1 day resulted in concentration-dependent uptake of the nanoparticles at $0.01-10 \mathrm{mg} / \mathrm{l}$. At $1-10 \mathrm{mg} / \mathrm{l}$ of the nanoparticles slight increase of water uptake by the seeds was observed. The increase of seed germination as well as of shoot and root dry 
weight was observed. The stimulatory effect decreased with nanoparticle concentration. The aim of the study was to elucidate the stimulatory effect of silver nanoparticles at low concentrations on wheat growth at early stages of ontogenesis. The nanoparticles were referred to as an example of a prolonged action form of a biogenic metal produced by means of 'green nanochemistry' and acting as a plant growth stimulant [61]. We have produced calcium alginate nanoparticles with gold core (Figure 2). At first, gold nanoparticles were synthesized by tetrachloroaurate reduction with polyethylene glycol using a method modified from [62]. After physical immobilization of sodium alginate onto their surface and resuspending into water $\mathrm{CaCl}_{2}$ solution was added. The reaction protocol must be followed very thoroughly in order to avoid extensive aggregation and destabilization of the nanoparticles. The size of the nanoparticles can be adjusted by means of auto metallography. Selection of other volumes of water and $\mathrm{CaCl}_{2}$ solution allowed us to produce stable aggregates [63].

An interesting dependency of the thickness of a layer of physically adsorbed sodium alginate on magnetite $\left(\mathrm{Fe}_{3} \mathrm{O}_{4}\right)$ nanoparticles expressed as particle size on experimental conditions was revealed. The average diameter of the nanoparticles dripped onto a TEM grid was 70nm. But if the same nanoparticles (without their separation from alginate solution) were sprayed onto the grid, their average size became $14 \mathrm{~nm}$. Aerosolization of the same core magnetite nanoparticles mixed with sodium alginate solution of another concentration into $\mathrm{CaCl}_{2}$ solution led to nanoparticles with average diameter of $8 \mathrm{~nm}$. If chitosan and sodium tripolyphosphate were used instead of alginate and $\mathrm{CaCl}_{2}$, the sizes of the resulting nanoparticles were also unequal in the three cases. The nanoparticles were intended for drug delivery or hyperthermia therapy of cancer [64].

By mixing a solution containing $\mathrm{CaCl}_{2}$, doxorubicin hydrochloride and a plasmid encoding p53 with another solution containing sodium carbonate and sodium alginate, nanoparticles containing simultaneously DNA and doxorubicin were prepared. Due to the existence of alginate the growth of $\mathrm{CaCO}_{3}$ based coprecipitates

\section{Emulsification-Internal Gelation}

could be effectively retarded. The resulting nanoparticles had size about $145 \mathrm{~nm}$ and zeta potential $-14 \mathrm{mV}$, and these parameters for the nanoparticles without alginate were $300 \mathrm{~nm}$ and $-2.5 \mathrm{mV}$, respectively. The encapsulation efficiency was $89 \%$ for DNA and $84 \%$ for doxorubicin. Loading efficiencies were $10 \%$ and $1 \%$, respectively. About $40 \%$ of encapsulated doxorubicin were released in water for 48 hours with initial burst release. Proliferation of HeLa cells exposed to the nanoparticles at $37^{\circ} \mathrm{C}$ for 48 hours was completely inhibited, and apoptosis was induced. Therefore, they have promising applications in cancer treatments. Cell inhibition rate was dose-dependent at $0.1-0.4 \mu \mathrm{g} / \mathrm{ml}$ of doxorubicin. It was always higher for encapsulated doxorubicin. The nanoparticles containing $2 \mu \mathrm{g}$ of DNA displayed slightly higher cell inhibition rate than those containing $1 \mu \mathrm{g}$ of DNA. At $0.2 \mu \mathrm{g} / \mathrm{ml}$ of doxorubicin most HeLa cells became rounded or detached from the plate, and blebbing occurred. The nanoparticles without alginate led to lower cell inhibition rates. If the nanoparticles contained another plasmid (pGL3-Luc) and did not contain doxorubicin, no cytotoxicity was found [65]. Layer-by-layer absorption of alginic acid and protamine in water or $0.5 \mathrm{M} \mathrm{NaCl}(\mathrm{pH} 5.5)$ onto spherical calcium carbonate microparticles produced in the presence of alginic acid and having size from 2 to $12 \mu \mathrm{m}$ with subsequent dissolution of the $\mathrm{CaCO}_{3}$ core in $\mathrm{HCl}$ or EDTA was also reported. The microcapsules synthesized in this way could be used for the encapsulation of polymers and their $\mathrm{pH}$-dependent release, as demonstrated for dextrane conjugated with fluorescein isothiocyanate [66].

Although not stated in the respective publications, the great advantage of this method is the improved particle size control. For example, the techniques of synthesis of metal nanoparticles with predictable size already exist. Another advantage is the possibility of encapsulating the substances into some types of core particles instead of encapsulation into the alginate layer. In this case the alginate layer acts as an additional diffusional barrier. But the existence of a core particle limits versatility and the possibility of food or biomedical application of these particles. However, this is a convenient method of generating micro- or nanocapsules by chemical dissolution of some types of core particles.

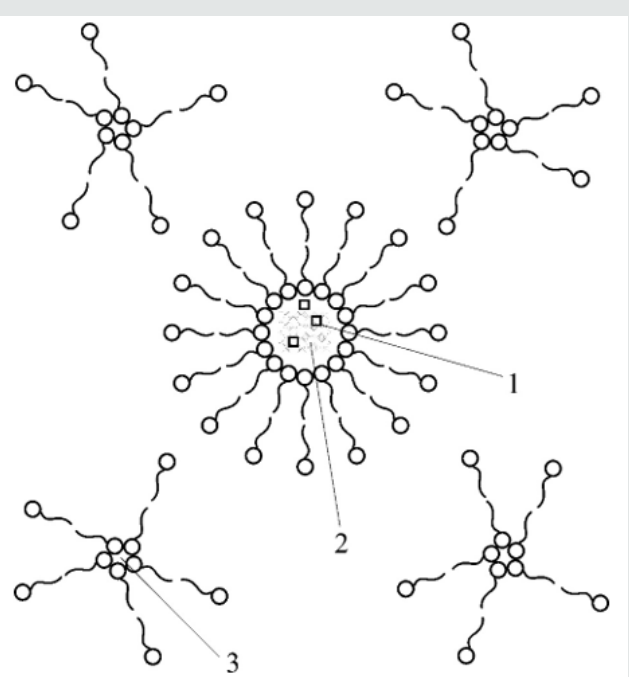

Figure 3: Alginate particle prepared using emulsification method. 1 - encapsulated drug; 2 - alginate core; 3 - empty micelle. 
In this method, an aqueous solution of sodium alginate containing an insoluble calcium salt (usually carbonate) is emulsified into an oil phase (Figure 3). Upon addition of an acid, calcium ions are liberated inside the alginate drops converting sodium alginate into calcium alginate, i.e. to a hydrogel, and the drops become being particles.

The technique of emulsification-internal gelation was useful for glucose oxidase encapsulation. A water-in-oil emulsion consisting of mineral oil, surfactant Span 80 , acetic acid and sodium alginate solution - previously ultrasonicated with calcium carbonate was prepared. Then water was added, the emulsion was stirred, and the formed beads were rinsed with Tween 80 and water. The resulting empty beads were incubated in buffered enzyme solution followed by chitosan coating. In the wet state the microparticles had volume-based mean diameter of $138 \mu \mathrm{m}$. After lyophilization, storage at $-20{ }^{\circ} \mathrm{C}$ and rehydration the immobilized enzyme lost $10 \%$ of its activity, and the uncoated beads collapsed. Loading and encapsulation efficiencies were $10 \%$ and $80 \%$, respectively, and depended on enzyme loading $\mathrm{pH}$. Loading efficiency increased with enzyme concentration, while encapsulation efficiency did not. Both parameters decreased with chitosan concentration, but the activity of the immobilized enzyme reached a maximum peak at $0.5 \%$ chitosan. It was concluded that these nanoparticles had the potential to serve as a useful and efficient delivery system for therapeutic proteins. Interestingly, at high enzyme concentrations the immobilized enzyme exceeded in activity the free one [67]. In a related report, no coating was applied, and canola oil was used as oil phase. Dry $\mathrm{CaCO}_{3}$ powder was ground prior to application to decrease grain size from $30 \mu \mathrm{m}$ to $2.5 \mu \mathrm{m}$. $\mathrm{CaCO}_{3}$ substitution for oxalate or tartrate was unacceptable because too low $\mathrm{pH}$ was required. Otherwise, phosphate was rejected because of too large grain size, and citrate produced large beads with a high variability in size distribution. At $\mathrm{CaCO}_{3} /$ alginate monomer ratios higher than $1 / 4$, residual insoluble calcium salt was observed inside the particles. The alginate particles had mean diameters from 50 to $1000 \mu \mathrm{m}$. The use of low guluronic and low or medium viscosity alginate gave a narrowest particle size distribution, but high guluronuc content and homopolymer blocks led to stronger gels. If Span 80 concentration increased up to $1 \%$, the mean particle size decreased asymptotically. The particles were stronger and more spherical when using larger amounts of acetic acid while limiting the $\mathrm{pH}$ reduction by enhancing the buffer capacity of sodium alginate solution using carbonate buffer. Further reduction of polydispersity was achieved by dropping the oil phase into the acidic buffer solution in water. The synthesis can be carried out under sterile conditions, and its potential application may be cell immobilization [68]. A similar protocol did allow the encapsulation of mouse insulinoma 6 cells. For this, to the sodium alginate solution containing a suspension of $\mathrm{CaCO}_{3}$ and cells in their culture medium mineral oil was added followed by the addition of acetic acid previously mixed with mineral oil. After that, a buffered saline solution mixed with culture medium was added to neutralize the $\mathrm{pH}$. All the experiment was carried out under agitation, and its rate determined the particle size. It could be made from $\sim 200$ to $2000 \mu \mathrm{m}$ (circularity was 0.9 ), the distribution was close to normal but very wide. The beads were separated by centrifugation, washed and separated by filtration. $\mathrm{CaCO}_{3}$ was not completely dissolved, and its grains remained inside the alginate beads. The beads had lower compressive stress resistance than those produced by extrusion using a commercial encapsulator. MOPS buffer provided better cell survival than HEPES buffer under optimized conditions ( $\mathrm{pH}$, buffer concentration, emulsification and acidification time). Cell division inside the beads was observed, and the encapsulated cells produced insulin at higher concentrations than single cells. The emulsion process is potentially suitable for cell therapy and transplantation purposes [69]. The use of paraffin oil has also been reported. In this case, a homogenized $\mathrm{CaCO}_{3}$ suspension in a solution containing sodium alginate, chitosan and drug (salbutamol sulfate) was added into liquid paraffin oil containing Span 80. After emulsification, liquid paraffin containing glacial acetic acid was added, and stirring was continued until $\mathrm{CaCO}_{3}$ was solubilized. Then $\mathrm{CaCl}_{2}$ solution containing Tween 20 was added to recover the beads by decantation. Beads were washed with the same solution and then with acetate buffer. The parameters for the optimized formulation were as follows: spherical, size $591 \pm 40 \mu \mathrm{m}$, encapsulation efficiency $95 \%$. These values increased conforming to increasing polymer concentration. Cumulative drug release after 2 hours at pH 1.2 was 7\%, after subsequent 3 hours at pH 6.8 (phosphate buffer) 10\%, and after 5 hours at pH 7.4 (phosphate buffer) $99.6 \%$. It was concluded that the hydrogel particles were the potential system for colon delivery of salbutamol sulphate via oral route for chronotherapy of nocturnal asthma [70].

However, in another publication [71] it was stated than salbutamol sulfate absorption site was not colon, moreover, it would degrade in colon, although no data was presented. Using the same technique as that in the previous report, alginate microparticles having mean size from 4 to $7 \mu \mathrm{m}$ (depending on recovery protocol) with immobilized insulin were synthesized. The particles were recovered from the oil phase by washing it with acetate buffer and separated by decantation. The remaining particles were recovered by centrifugation. The difference between the recovery protocols were centrifugation rate and additives to the buffer ( $\mathrm{CaCl}_{2}$, Tween 80 , solvents). Only the protocol employing acetone, isopropanol and hexane retained the secondary structure of insulin. The encapsulation efficiency was more than $75 \%$ with the particle recovery yield around $70 \%$. The particles were uniform and spherical with a rough surface. It should be noted that all the formulations showed burst release of all the encapsulated insulin for $5 \mathrm{~min}$ in simulated gastric solution without enzymes. But the technology was claimed as promising for insulin delivery due to the gentle methodology of producing microparticles [72]. In order to evaluate the effectiveness of insulin-loaded nanoparticles on its permeability across intestinal cells, another encapsulation technique was used. Here the alginate solution contained dextran sulfate, poloxamer 188 and ultrafine calcium carbonate suspension. Sorbitan monooleate was used for emulsification of this solution into paraffin oil under stirring and ultrasonication. Insulin solution in $\mathrm{HCl}$ was added simultaneously with acetic acid solution in oil under the same conditions. Nanoparticles were washed and coated with chitosan, polyethylene glycol and albumin. At the time of 
coating they were treated with $\mathrm{CaCl}_{2}$. Their average size was $300 \mathrm{~nm}$ with polydispersity index 0.17 , zeta potential $29 \mathrm{mV}$, encapsulation efficiency $31 \%$ and loading efficiency $6 \%$. The nanoparticles released up to $35 \%$ of insulin at $\mathrm{pH} 1.2$ for 2 hours. At pH 5.5 burst release was observed. Dye-labeled nanoparticles applied at $0.5 \mathrm{mg} / \mathrm{ml}$ were found at the cell membranes and probably inside Caco-2 and HT29-MTX cells co-cultured in a 90:10 proportion. Nevertheless, at $0.1-1 \mathrm{mg} / \mathrm{ml}$ they had relatively low cytotoxicity for separately cultured AGS, Caco-2 and HT29-MTX cells. At 0.5 $\mathrm{mg} / \mathrm{ml}$ the nanoparticles enhanced insulin permeation through a monolayer of Caco-2 and HT29-MTX cells co-cultured with Raji B cells (a model of intestinal wall) from the apical to basolateral direction at $\mathrm{pH} 5.5$ and especially at $\mathrm{pH}$ 7.4. Insulin permeability was demonstrated to be mediated by clathrin endocytosis. The clinical potential of the nanoparticles for the oral delivery of insulin and therapy of type I diabetes mellitus was envisaged [73].

Internal gelation using canola oil containing acetic acid and surfactant Span 85, and sodium alginate solution containing $\mathrm{CaCO}_{3}$ nanoparticles (116-244nm depending on the conditions, zeta potential $14 \mathrm{mV}$ ) capped with surfactant AOT carried out in an electro dispersion reactor allowed size control of the microparticles by peak voltage increase in the range of 412 to $10 \mu \mathrm{m}$. Further peak voltage increase did not decrease the bead size substantially, i.e. the method is currently limited to microparticles. Dried particles aged in $\mathrm{CaCl}_{2}$ solution had a diameter of about $5 \mu \mathrm{m}$. The flow rate ratio also significantly influenced the droplet size. The particles were highly porous because of $\mathrm{CO}_{2}$ release during their formation and were spherical with a wrinkled surface. Only a slight amount of $\mathrm{CaCO}_{3}$ was required to induce gelation, viz. $0.01 \mathrm{M}$ for $4 \%(\mathrm{w} / \mathrm{v})$ alginate, although much higher concentrations were used in practice. The microparticles appeared suitable for encapsulation of pharmaceutical compounds [74].

A technique of internal gelation without employing emulsification has been reported. Mixing $\mathrm{CaCl}_{2}$ with DM-nitrophen ${ }^{\mathrm{TM}}$ leads to a complex. This complex has a $\mathrm{K}_{d}$ of $5 \mathrm{nM}$ that dramatically increases after UV photolysis. Bulk hydrogels prepared in this way were intended for patterned microscale 3D cell culture, and their applicability for human cell encapsulation was demonstrated. But the high cost of DM-nitrophen ${ }^{\mathrm{TM}}$ limits its applicability [75].

In a previous review [76] the following advantages of emulsification-internal gelation method were stated:

- Particle size can be easily controlled from a few nanometers (potentially) to millimeters by controlling oil viscosity, sodium alginate type and concentration, surfactant concentration, and emulsification conditions, including mixing.

- $\quad$ The particles are homogeneous.

- Low shear protects fragile encapsulants.

- $\quad$ Scale-up potential is almost unlimited without the loss of particle size control

The following disadvantages were pointed out:

- High porosity of the particles.
- Possible loss or inactivation of encapsulated proteins during formulation and washing steps

\section{Additional concerns:}

- If mineral oils (paraffin oil, isooctane, silicon oil, etc.) are used, they may cause environment-related problems.

- Acid concentration must be carefully calculated to avoid overdosing [76].

\section{External Gelation}

In this method a soluble calcium salt (usually chloride) induces alginate gelation. But simple mixing the solutions of sodium alginate and $\mathrm{CaCl}_{2}$ yields bulk hydrogel without any particles. Therefore, special techniques are used.

\section{Emulsification coupled with external gelation}

Here sodium alginate solution containing a drug being encapsulated is emulsified in an oil phase. The emulsion droplets formed act as templates for the formation of spherical particles. Then $\mathrm{CaCl}_{2}$ solution is added converting the drops to particles [77].

Surfactants are usually used to stabilize the emulsions. However, sometimes they are not employed. The addition of sodium alginate solution to sunflower oil with subsequent homogenization by mixing, addition of $\mathrm{CaCl}_{2}$ solution and acetic acid and another homogenization step led to beads. They were separated by centrifugation, washed with hexane and water followed by treatment with isopropanol: water mixtures with increasing concentration of isopropanol up to $100 \%$. The last step was supercritical drying. The loading was carried out by bead incubation at $40{ }^{\circ} \mathrm{C}$ at 120 atm with ibuprofen for 24 hours. Bead characteristics were as follows: loading efficiency $30.5 \%$, pore size $35 \mathrm{~nm}$, surface area $359.5 \mathrm{~m}^{2} / \mathrm{g}$. High loading efficiency makes these aerogel beads efficient drug carriers [78].

In a simple technique employing a surfactant, $\mathrm{CaCl}_{2}$ solution was added to that of sodium alginate containing 5-25\% PEG-1500 or $0.5-5 \%$ Tween 80 at pH 5 under sonication. The nanoparticles were separated from the reaction mixture by centrifugation after 24 hours $[66,79]$. They had average diameter of $210-540 \mathrm{~nm}$ but were prone to collapse during drying [66]. The amount of PEG1500 and Tween 80 was about $64 \mathrm{mg}$ and $19 \mathrm{mg}$, respectively, per one gram of lyophilized nanoparticles independently of the initial concentrations of these stabilizers in sodium alginate solution. In the presence of Tween 80 some hydrophobic regions were formed inside the nanoparticles. The nanoparticles containing polyethylene glycol on the surface are expected to have low immunogenicity, and those having Tween 80 are expected to cross blood-brain barrier [79].

Nanoparticles were also produced by dropwise addition of sodium alginate solution containing a plasmid encoding enhanced green fluorescent protein to the solution of AOT in toluene. Then $\mathrm{CaCl}_{2}$ solution was added. After washing with acetone and water the nanoparticles were separated by centrifugation. They had a core consisting of sodium alginate, and a membrane consisted of calcium alginate. Their morphology depended on the position on 
the respective point on the phase diagram, and that was in turn determined by the toluene: alginate: AOT ratio. Only in one region separated and spherical nanoparticles having size $55-100 \mathrm{~nm}$ (average size $80 \mathrm{~nm}$ ) were formed. Transfection of cultured NIH 3T3 cells performed using the nanoparticles (at $2 \mu \mathrm{g} / \mathrm{ml}$ of encapsulated DNA) and the complex of DNA with polyethyleneimine has shown comparable results after 48 hours, but the nanoparticles performed more slowly. The nanoparticles were proposed as non-viral vectors for gene therapy. Once internalized by non-phagocytic cells via endocytosis pathways, they could undergo quick erosion and elicit osmotic swelling, hence facilitating endosomal escape of gene to the cytoplasm [80].

Alginate microparticles can be used as a source of $\mathrm{Ca}^{2+}$ ions causing gelation of sodium alginate solution (in phosphate buffered saline) they were placed into [81]. The latter solution may contain peptides [81,82], oligonucleotides [81] or cells to be delivered by subcutaneous injection close to a tumor site. The solution had a volume of $150 \mu \mathrm{l}$ and contained $\sim 10^{6}$ microparticles. Gelation in vivo occurred for several minutes. The gels remained intact in mice for more than 21 days and than dissolved [81]. The peritumoral gels carrying antigen-pulsed dendritic cells accumulated CD4+ ${ }^{+}$-cells but did not attract a significant amount of CD8 ${ }^{+} \mathrm{T}$-cells, and the effect on established tumor growth was limited. If the gel also contained interleukin-15 super agonist, it could be released for over 2 weeks in vitro (in RPMI growth medium) and for 5-7 days in vivo. This gel retarded tumor growth and caused transient reduction of the size of larger tumors. This effect was mainly caused by the cytokine, with dendritic cells providing only a minor effect. The average suppression of tumor growth caused by the above-mentioned gel, the same gel without the cells but containing the cytokine and Tolllike receptor-9 agonist $\mathrm{CpG}$ and the gel containing only the cytokine but injected two times was equal. Local immunotherapies may be used for treatment of unresectable tumors and sites of surgical resection to combat local recurrence. Local immune response might make the tumors to become in situ vaccines themselves [82]. The microparticles with the average diameter of $20 \mu \mathrm{m}$ were synthesized as follows. Sodium alginate solution in phosphate buffered saline was added dropwise to the solution of Span 80 and Tween 80 in isooctane. After homogenization of the resulting solution $\mathrm{CaCl}_{2}$ solution was added followed by another homogenization step, centrifugation and washing with isooctane and water [81].

A close technique can be used to form alginate nanocapsules. Nanocapsules are vesucular systems having oily or aqueous core surrounded by a polymeric membrane. The drug is mixed with an organic solvent, and the mixture is slowly added to aqueous solution of sodium alginate containing a surfactant, and an oil-inwater emulsion is formed under sonication. Finally, $\mathrm{CaCl}_{2}$ solution is slowly added, and resulting calcium alginate forms a drop membrane. This method is reviewed in [77].

Sometimes liquid paraffin can be used as an oil phase. The solution containing guar gum, sodium alginate, Tween 80 and drug (salbutamol sulfate) was added dropwise to liquid paraffin followed by the addition of $\mathrm{CaCl}_{2}$ solution and incubation. Another preparation was produced by dropwise addition of a solution containing chitosan, sodium alginate, the drug and acetic acid to liquid paraffin followed by addition of dioctyl sodium sulfosuccinate and of glutaraldehyde solution in toluene. All the experiments were carried out under stirring. The microparticles produced were filtered, washed with petroleum ether and dried. They were spherical with average size of about $438 \mu \mathrm{m}$. Encapsulation efficiency was more than 95\%. Their incubation in simulated gastric fluid for 2 hours and subsequently in simulated intestinal fluid for 10 hours caused gradual release of more than $85 \%$ of the encapsulated drug. Salbutamol sulfate is used in the management of bronchopulmonary diseases involving bronchospasm, and its sustained release dosage forms are needed to reduce frequent dosing (3-4 times a day) [83]. Interestingly, a close technique without the use of a surfactant was reported. Salbutamol sulfate and liquid paraffin were added to a solution containing sodium alginate, hydroxypropyl methylcellulose and starch. Homogenization and stirring led to a stable emulsion that was extruded through a needle into $\mathrm{CaCl}_{2}$ solution and incubated in it followed by filtration, washing and drying. The beads were mixed with magnesium stearate and manually filled into empty hard gelatin capsules. The capsules were administered to fasted rabbits through plastic tubing followed by flushing of water. They remained in the stomach for more than 6 hours. Their floating time in simulated gastric fluid in vitro was also more than 6 hours. The capsules used for the experiment with rabbits also contained $\mathrm{BaSO}_{4}$. Encapsulation efficiency and bead size increased with hydroxypropyl methylcellulose and starch amount increase. This might be due to emulsion viscosity increase. Bead density decreased with hydroxypropyl cellulose amount increase and starch amount decrease. Drug release after 10 hours of capsule floating in simulated gastric fluid decreased simultaneously. For the optimized formulation encapsulation efficiency and drug release were $70 \%$ and $57 \%$, respectively, with the average bead diameter of $1.9 \mathrm{~mm}$ and density of $0.85 \mathrm{~g} / \mathrm{cm}^{3}$. Bead surface was rough and had small pores. These stomach-specific floating systems might be beneficial over the conventional oral dosage forms (prone to tolerance development after repeated administration) because of prolonged gastroretentive delivery of drugs with improved bioavailability, therapeutic efficacy and patient compliance [84].

In order to ensure production of calcium alginate nanoparticles without damaging the biomolecules being encapsulated, reversed phase inversion temperature method has been proposed. In this case, the mixture of aqueous sodium alginate solution, decane and tetra ethylene glycol monododecyl ether employed as a surfactant was equilibrated at the temperature slightly below the phase inversion temperature of this system. Then the mixture was quickly transferred to a temperature well above it leading to formation of water-in-oil emulsion, and $\mathrm{CaCl}_{2}$ addition to this emulsion led to the formation of nanoparticles. The most stable particles were produced if $\mathrm{CaCl}_{2}$ was added as a simple solution, not as waterin-oil emulsion. All the synthesis was carried out under stirring. In order to separate the nanoparticles, decane was added leading to precipitation of micrometer-sized structures with an irregular shape. The nanoparticles remaining in solution were spherical and homogeneous with mean radius of $50 \mathrm{~nm}$ and size distribution $\sim 20-120 \mathrm{~nm}$ in radius. The yield of nanoparticles was $\sim 18 \%$. The 
amount of the surfactant and decane remaining in the sample was below 0.01 wt. $\%$ and 0.2 wt. $\%$, respectively. The procedure has potential to be scaled up and to be adapted for use with pharmaceutically acceptable oils. The size of the resulting particles makes them suitable for delivery of biomacromolecules to cells and certain mucosal tissues [85].

In an approach developed at the same time by another group, both sodium alginate (containing bovine serum albumin as a model protein) and $\mathrm{CaCl}_{2}$ aqueous solutions were emulsified in isopropyl myristate using dioctyl sodium sulfosuccinate as a surfactant. $\mathrm{CaCl}_{2}$ microemulsion was poured dropwise into the alginate one followed by ultrasonication. The nanoparticles were separated by centrifugation and washed with acetone. The size of the particles was about $500 \mathrm{~nm}$, but particles smaller than $100 \mathrm{~nm}$ were also present. Loading efficiency was $40 \%$. The nanoparticles showed burst release of the encapsulated protein into phosphate buffered saline for $15 \mathrm{~min}$, followed by a plateau and than by sustained release of all the remaining protein for more than 8 hours. The released protein did not suffer covalent aggregation or degradation by fragmentation. The nanoparticles were intended for oral protein drug delivery, and the components for their preparation were pharmaceutically acceptable [86].

A possibility to produce alginate particles without using water has been reported. This technique involves addition of mixture of drug (salbutamol sulfate), sodium alginate, $\mathrm{CaCl}_{2}$ and PEG-400 into molten PEG-6000. All the reagents (except PEG-6000) were applied as powders. The resulting mixture was cooled rapidly at $-20{ }^{\circ} \mathrm{C}$ and then powdered in a mortar followed by sieving. Particle size range for different formulations was $6.75-9.8 \mu \mathrm{m}$. They had rough surface [87]. Interestingly, particle size increase with sodium alginate concentration increase reported for encapsulation of the same drug by dripping method [88] was observed here as well. Drug release in phosphate buffer decreased with sodium alginate concentration increase and increased with PEG-6000 proportion decrease. This was explained by hydrogen bonds between alginate and polyethylene glycol. All the formulations tested showed burst release with subsequent release of the remaining drug for several hours. They were intended for pulmonary drug delivery by means of a dry powder inhaler [87].

The advantages of the method are the same as those stated above for emulsification-internal gelation, except particle homogeneity. The additional advantages are easier formulation because of no need for finely dispersed insoluble calcium salt, decreased porosity and the absence of acid. The disadvantages are the use of oil and usual need for surfactants with questionable biocompatibility. Therefore, these nanoparticles have rather low chances for food applications, but limited agricultural or even biomedical use is possible. It should be noted, however, that surfactants make the nanoparticles less prone to aggregation. Therefore, the nanoparticles produced by this method must have enhanced stability.

\section{Microfluidic devices}

The advances in microfluidics paved the way to its application in nanotechnology, the synthesis of calcium alginate nanoparticles not being an exception. The parts of microfluidic chips can be manufactured in the laboratory from plastic by means of commercially available computer-controlled laser cutters.

For the synthesis of alginate microparticles having diameters in the range $50-2000 \mu \mathrm{m}$ with a variation less than $5 \%$ a microfluidic chip has been invented. A stream of sodium alginate solution was focused by two sunflower seed oil streams leading to emulsion that was dripped into $\mathrm{CaCl}_{2}$ solution, and microparticles formed and precipitated at the interface between the phases. The emulsion size increased when the aqueous flow increased. When the oil flow increased, the emulsion size decreased. Increasing both oil and alginate solution flow rates while maintaining the flow rate ratio resulted in smaller emulsions. Gold nanoparticles were encapsulated into the alginate microparticles at the time of their synthesis. The microparticles were freeze-dried and than vacuumdried, but most of them retained spheroid shapes. The approach will provide many potential uses for pharmaceutical applications such as targeted drug delivery and DNA transfection [89]. In another microfluidic device liquid paraffin containing Span 80 was used as the continuous phase and sodium alginate solution containing $\mathrm{Na}_{2} \mathrm{SO}_{4}$ served as the dispersed phase. The droplets of the mixture were collected in $\mathrm{BaCl}_{2}$ solution. This led to the formation of barium alginate microspheres with $\mathrm{BaSO}_{4}$ nanoparticles inside. The microspheres were washed and stored in $\mathrm{NaCl}$ solution. They were spherical with the diameter 229-284 $\mu$ m (average $250 \mu \mathrm{m}$ ). Their size could be modulated by adjusting the flow rate ratio of the two phases. The distribution of $\mathrm{BaSO}_{4}$ nanoparticles was denser on the edge of the microspheres than in their interior. The aggregation of the nanoparticles was not observed. $\mathrm{BaSO}_{4}$ dispersion density increased with $\mathrm{Na}_{2} \mathrm{SO}_{4}$ concentration increase, but at $0.4 \mathrm{M} \mathrm{Na}_{2} \mathrm{SO}_{4}$ the precipitate appeared on the microsphere surface making it brittle. The microspheres were suggested as an embolic agent synthesized by means of droplet microfluidics and intended for transcatheter arterial embolization. In this method an embolic agent is injected through a microcatheter guided by a radiographical instrument in order to cause artery occlusion and to prevent nutrient supply to the tumor. At $5 \mathrm{mg} / \mathrm{ml}$ the microspheres were not cytotoxic to HepG2 cells. After injection of them at $\sim 30 \mathrm{mg} / \mathrm{ml}$ to the renal artery of healthy rabbits interlobular and arcuate arteries became almost completely filled with the microspheres possessing excellent X-ray visibility. These microspheres showed better and longer lasting X-ray visibility after embolization if compared with commercially available calcium alginate microparticles that did not contain $\mathrm{BaSO}_{4}$. The described microspheres present exciting opportunities for potential clinical applications in transcatheter arterial embolization therapy and for noninvasive tracking and mapping of the fate of the embolic agents in vivo [90].

Needless to say about the great advantages of microfluidic devices. They simplify scaling up while minimizing batch-to-batch variations and labor requirements. Moreover, they can be part of more complex devices and enable synthesis of nanoparticles in the field. The possibility to synthesize nanoparticles in small quantities is promising for personalized medicine. However, not every specialist can invent them, and it would be impossible for many poorly equipped or non-technical (e.g. purely biomedical) laboratories to construct them. 


\section{Water-in-oil-in-water emulsions}

In more complex variant of the technique, the water-inoil emulsion is further emulsified in water containing another surfactant, and then $\mathrm{CaCl}_{2}$ solution is added. Calcium alginate nanoparticles (500-700 nm, zeta potential around $-13 \mathrm{mV}$ ) stabilized by AOT and polyvinyl alcohol were synthesized by gradual addition of calcium chloride solution to water-in-oil-in-water emulsion of sodium alginate containing doxorubicin or rhodamine being encapsulated. Loading efficiency for these drugs was 5\% and 4\%, respectively. The nanoparticles enhanced doxorubicin cytotoxicity to cultured cancer cells in a dose-dependent manner (at 0.01-0.1 $\mu \mathrm{g} /$ $\mathrm{ml}$ for MCF-7 cells and at $0.1-10 \mu \mathrm{g} / \mathrm{ml}$ for NCI-ADR/RES cells) by extending its way to nucleus overcoming P-glycoprotein-mediated efflux without the use of additional P-glycoprotein inhibitors. At highest concentration of the encapsulated doxorubicin no viable cells were observed. Overexpression of P-glycoprotein results in the development of multidrug resistance of cancer cells, but drug encapsulation in nanoparticles overcomes this effect. Increased therapeutic efficiency was associated with an increase in the level of cellular and nuclear drug accumulation [91]. Similarly produced nanoparticles enhanced activity of encapsulated methylene blue in photodynamic therapy of cultured cancer cells (at $\sim 0.5-20 \mu \mathrm{M}$ in a concentration-dependent manner for MCF-7 cells) by promoting its nuclear delivery and increasing production of reactive oxygen species. Decreased viability of 4T1 cells was observed only at the highest drug and light doses (at $0.6 \mu \mathrm{M}$ and $2400 \mathrm{~mJ} / \mathrm{cm}^{2}$ but not at $0.3 \mu \mathrm{M}$ or at $1200 \mathrm{~mJ} / \mathrm{cm}^{2}$ ). The parameters of the nanoparticles were as follows: average diameter $79 \mathrm{~nm}$, surface charge $19 \mathrm{mV}$, encapsulation efficiency $82 \%$. Methylene blue can be used as a photosensitizer in photodynamic therapy of cancer, but its clinical use has been limited because of insufficient therapeutic efficacy. The respective study was aimed at enhancement of its efficiency by means of encapsulation in nanoparticles [92]. Lyophilized nanoparticles produced using the same technique and by the same group increased the efficiency of photodynamic therapy based on $8 \mu \mathrm{M}$ free or encapsulated methylene blue and directed against 4 cultured cancer cell lines under ambient conditions. They also reduced mammosphere formation by cancer stem cells present in the culture of MCF-7 cells under normoxia and hypoxia. It was achieved by switching the mechanism of radical generation to a type I reaction requiring much less molecular oxygen. This is significant because of a usually hypoxic tumor microenvironment. The loss of efficacy at low concentration of oxygen is a major limitation of photodynamic therapy, and it can be overcome by promoting type I chemistry by methylene blue encapsulation in nanoparticles composed of polymer and surfactant that are both anionic. The nanoparticles were spherical with a hydrodynamic diameter of $275 \mathrm{~nm}$, zeta potential of $-20 \mathrm{mV}$ and loading efficiency of $7 \%$. Aggregates were separated by centrifugation [93]. The AOT-alginate nanoparticle system is a promising delivery vehicle for anticancer photodynamic therapy with methylene blue $[92,93]$. The diameter of the freeze-dried nanoparticles with simultaneously encapsulated doxorubicin and methylene blue was $40 \mathrm{~nm}$ if measured by means of atomic force microscopy and $\sim 73 \mathrm{~nm}$ if measured using dynamic light scattering. Aggregates might form, but they were separated by centrifugation. Drug loading was $7 \%$ and $13 \%$, respectively. Their administration via a lateral tail vein to mice with drug-resistant mammary adenocarcinoma at 4 and $8 \mathrm{mg} / \mathrm{kg}$, respectively, followed by light exposure slowed down the tumor growth and increased mice survival. $15 \%$ of tumor cells were apoptotic compared with $1 \%$ if nonencapsulated drugs were administered. The nanoparticles also damaged tumor neovasculature. Nanoparticlemediated combination of chemotherapy and photodynamic therapy has significant therapeutic potential against drug-resistant tumors, for it could overcome drug resistance by invoking multiple anticancer mechanisms. Encapsulation of the drugs in alginate nanoparticles may result in significantly enhanced intracellular accumulation and production of reactive oxygen species [94]. Encapsulation efficiency of methylene blue could be increased up to $99.8 \%$ by sodium alginate and AOT concentration increase as well as by decrease of the volume of the AOT phase. But it decreased with the amount of drug added to the formulation. Many other 'model drugs' could also be encapsulated, for example verapamil, clonidine, diclofenac and fluorescein. The encapsulation efficiency and release profile strongly depended on drug composition. The release of weakly acidic diclofenac in phosphate buffered saline was faster than that of acidic doxorubicin and verapamil, but even the release of $70 \%$ of encapsulated diclofenac lasted for 7 days with initial burst release. The encapsulation efficiencies for these drugs were $6 \%, 60 \%$ and $77 \%$, respectively. Two drugs could be encapsulated simultaneously, but the release became faster. The zeta potential of the nanoparticles was in the range of -25 to $-35 \mathrm{mV}$. Surfactant-polymer nanoparticles would be useful for sustained delivery of water-soluble drugs, maybe together with imaging agents [95]. Similar technique was used for encapsulation of pesticide imidacloprid in order to investigate the ultimate effect of this pesticide in the form of nanoformulation. The nanoparticles had the following characteristics: size $\sim 150 \mathrm{~nm}$ (aggregates were present), encapsulation efficiency $99 \%$, loading efficiency $2.5 \%$. Increasing the concentration of AOT was found to increase particle size. Increasing the concentrations of both sodium alginate and AOT led to encapsulation efficiency increase, but the effect was complex. The encapsulated pesticide was less cytotoxic against cultured Vero cells at 5 and $10 \mu \mathrm{g} / \mathrm{ml}$ if compared to free imidacloprid. But it was more effective against leafhoppers after 11-15 days of spraying $150 \mathrm{ml}$ of its $0.145-\mathrm{mg} / \mathrm{l}$ solution on $\sim 10$ Okra (bhindi) plants growing on a total area of $1 \mathrm{~m}^{2}$. Nano formulations of pesticides will reduce the amount of pesticide needed, decrease the environmental pollution, side effects and the amount of pesticide residues in food. They will be beneficial for economy, be of increased safety for the farmer or pesticide applicator and offer targeted delivery [96].

As can be seen, the double emulsion technique is much less popular than those involving water-in-oil emulsions. They share the same disadvantages, but the former one is more labor-consuming and requires two surfactants. However, the technique may provide somewhat better particle size control, because the maximum size of the aggregates is limited by the size of the micelle. 


\section{Dripping method}

The simplest way to produce particles using external gelation is dripping one solution into another one (Figure 4). When a drop of sodium alginate solution faces $\mathrm{Ca}^{2+}$ ions, gelation immediately occurs onto its surface preventing its dissolution. Then $\mathrm{Ca}^{2+}$ ions slowly diffuse inside the drop to induce gelation in all its volume, and the drop becomes a particle.

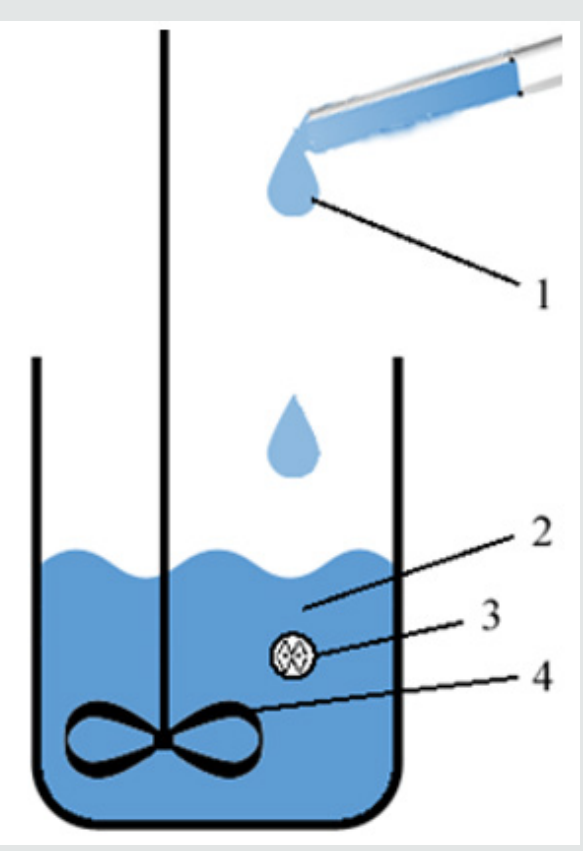

Figure 4: Dripping method. 1 - sodium alginate solution; $2-\mathrm{CaCl}_{2}$ solution (or vice versa; a drug can be in either solution); 3 - alginate particle; 4 - stirrer.

\section{Dripping $\mathrm{CaCl}_{2}$ into sodium alginate}

If $\mathrm{CaCl}_{2}$ solution is being dripped into sodium alginate solution, so-called pre-gel is initially formed. This pre-gel is a colloidal solution (i.e. nano- or microparticles) of calcium alginate hydrogel that has not separated into a new bulk phase yet. This separation (i.e. aggregation) can be prevented by addition of chitosan or other stabilizers. However, the optimization of reaction time (before separation of the particles) and of concentrations of $\mathrm{CaCl}_{2}$ and sodium alginate makes possible the synthesis of nanoparticles even without stabilizers.

The nanoparticles prepared by dropwise addition of calcium chloride solution to the sodium alginate solution under continuous ultrasonication had hydrodynamic diameter from 210 to $540 \mathrm{~nm}$. In the presence of stabilizers (Tween 80 or PEG-1500) smaller nanoparticles having improved stability were produced. The size distribution and stability of the nanoparticles were also affected by ageing in the supernatant. Using these nanoparticles, bovine serum albumin encapsulation efficiency of more than 99\% was demonstrated. The protein was admixed to alginate solution before the preparation of nanoparticles. The nanoparticles with encapsulated protein were claimed as perspective vehicles for hydrophobic drugs because they were composed only of components listed in the FDA Inactive Ingredients Database [97]. Another report in which a close technique was used dealt with insulin admixed to $\mathrm{CaCl}_{2}$ solution, and the nanoparticles were covered with chitosan. The main objective of the investigation was to develop insulin-loaded chitosan-coated alginate nanoparticles with small size in order to improve insulin bioavailability after oral administration. The parameters of the nanoparticles were as follows: average size $104 \mathrm{~nm}$, encapsulation efficiency $78 \%$, zeta potential $4 \mathrm{mV}$. These values increased with the amount of chitosan. $27 \%$ of insulin was released in simulated gastric fluid for 2 hours with initial burst release. When transferred in simulated intestinal fluid, the nanoparticles showed sustained and prolonged insulin release. Administered orally through a feeding needle at 50 or $100 \mathrm{IU} / \mathrm{kg}$ body weight, the nanoparticles demonstrated significant reduction in glycemia in diabetic mice for at least 9 hours with $\sim 8 \%$ insulin bioavailability. The nanoparticles were firmly attached to the mouse intestinal lumen, as shown ex vivo using freshly excised small intestinal tissue. The acute toxicity study revealed safety of the nanoparticles administered orally at $150 \mathrm{mg} / \mathrm{kg}$. No mortality, no hepatotoxicity (tested as serum levels of alanine aminotransferase, aspartate aminotransferase, glutamate oxaloacetate transaminase and lactate dehydrogenase) and no nephrotoxicity (tested as serum and urine creatinine as well as urine protein, urobilinogen, blood, ketone, bilirubin and glucose levels, $\mathrm{pH}$ and specific gravity) were observed. Pathohistological study also showed no damage to liver and kidney tissues. Hence, the nanoparticles might be employed as a promising carrier for oral delivery of therapeutic proteins [98]. Sometimes this encapsulation technique can be used even without ultrasonication. An optimization study in which sodium alginate was tested in the concentration range $0.07-0.75 \%(\mathrm{w} / \mathrm{v})$ and $\mathrm{CaCl}_{2}$ in the range of $0.025-0.15 \%$ $(\mathrm{w} / \mathrm{v})$ revealed their concentrations of $0.3 \%$ and $0.1 \%$, respectively, as optimal, i.e. leading to spherical nanoparticles without aggregates or bulk gel. The nanoparticles were incubated in their original solution under stirring followed by centrifugation and freezedrying. Incubation time and the stirring speed were also optimized. The nanoparticles coated with poly-L-lysine were used for bovine serum albumin immobilization. The protein was admixed to sodium alginate solution. Encapsulation and loading efficiencies increased with the protein concentration in sodium alginate solution up to $2 \mathrm{mg} / \mathrm{ml}$ and reached $76 \%$ and $73 \%$, respectively. The characteristics of protein-loaded nanoparticles were as follows: average size $50 \mathrm{~nm}$, polydispersity 0.36 and zeta potential $-24 \mathrm{mV}$. Protein release in phosphate buffered saline accelerated with time until 3 days, and finally $\sim 60 \%$ of it was released. The nanoparticles were suggested as a carrier for drug and vaccine delivery, and their properties strongly depended on concentrations of both sodium alginate and $\mathrm{CaCl}_{2}$ [99]. A close technique was used to produce nanoparticles loaded with zinc-insulin complex. The nanoparticles were covered with chitosan that was added dropwise to the resulting solution. They were spherical but had rough surface. Their size increased with decrease of alginate: chitosan mass ratio from 764 to $2209 \pm 496 \mathrm{~nm}$. pH decrease from 5.2 to 4.7 slightly decreased the mean particle size, but further decrease to 4.2 displayed the opposite effect. The formation of new chemical 
entities inside the nanoparticles was revealed, i.e. the nanoparticles with encapsulated insulin consisted of the complex and not of the physical mixture of the components [100]. In another case, insulin modified with methyl polyethylene glycol was added to sodium alginate solution, and then $\mathrm{CaCl}_{2}$ and chitosan were applied. The nanoparticles were incubated in the reaction mixture under stirring followed by centrifugation. At pH 1.2 the release of encapsulated insulin began after 20 minutes, at pH 5.6-7.2 65-90\% of insulin were released for 5 minutes. Therefore, the encapsulated insulin would mainly release in small intestines with minimal loss in the stomach [101]. The characteristics of chitosan-coated nanoparticles with encapsulated pesticide acetamiprid prepared in a similar way but with $\mathrm{pH}$ adjustment of the solutions were as follows: size 30$40 \mathrm{~nm}$, hydrodynamic diameter $201.5 \mathrm{~nm}$, polydispersity index 0.39 , zeta potential $-32 \mathrm{mV}$, encapsulation efficiency $62 \%$, maximum release $93 \%$. The nanoparticles were found to be superior to commercial formulation in terms of controlled release of the insecticide in soil and protecting it from degradation. The pesticide release rate increased with $\mathrm{pH}$ up to $\mathrm{pH} 10$. This formulation could be used in any type of soil. It could reduce the frequency of application of pesticides by controlling the release and subsequently reduce harm caused to health by handling of pesticides or through residues in food or environment. The cost of pesticide application would also be diminished because less pesticide would be needed, and alginate and chitosan are inexpensive [102]. Chitosan-coated alginate nanoparticles with encapsulated herbicide paraquat dichloride were prepared similarly. Interestingly, the particles were not removed from $\mathrm{CaCl}_{2}$ solution after preparation. Their characteristics were as follows: spherical, size 197-305 nm, hydrodynamic size $635 \mathrm{~nm}$, polydispersity index 0.52 , zeta potential $-23 \mathrm{mV}$, encapsulation efficiency $74 \%$. The encapsulation decreased paraquat soil sorption and was therefore expected to greatly improve its herbicidal action. The formulation was shown to be promising for agricultural use, offering increased duration of herbicide action, while reducing problems of environmental toxicity. The biodegradability of the nanoparticles is an additional advantage [103]. Likewise, prolonged release of herbicides imazapic and imazapyr from alginate nanoparticles covered with chitosan was shown. The aim of the work was to prepare and characterize nanoparticles containing these two pesticides simultaneously for use in agricultural weed control. The nanoparticles (average size 378 or $247 \mathrm{~nm}$ (aggregates were present), polydispersity index $\sim 0.3$, zeta potential $-30 \mathrm{mV}$, encapsulation efficiency $62 \%$ for imazapic and $71 \%$ for imazapyr) were produced using the same technique as in the above cases. The particles were not separated from their original solution, and remained stable in it for at least 30 days. At concentration equivalent to $400 \mathrm{~g} / \mathrm{ha}$ the encapsulated herbicides retained their activity against Bidens pilosa in soil, if applied pre-emergence. The parameters analyzed were germination efficiency, height, root length and green mass. The nanoparticles showed reduced genotoxicity to Chinese hamster cells treated with $0.1 \mathrm{mg} / \mathrm{ml}$ of the herbicides for 1 hour, as shown by comet tests, and to Allium cepa roots treated with $0.5 \mathrm{mg} / \mathrm{ml}$ of nanoparticles for 24 hours, as shown by relative alteration index determined using optical microscopy. At 75g/l of imazapic and 25g/l of imazapyr they also displayed reduced negative effect against soil bacteria 7 days after treatment, as revealed by real-time polymerase chain reaction (qPCR). After 30 days the result with the nanoparticles showed the greatest similarity to the negative control (untreated soil). The formulation offers an effective alternative for weed control in agricultural cultivations. The encapsulation of two herbicides in one carrier system to improve their activity and to reduce the impact on humans and environment was proven to be a good strategy for sustainable agricultural production, although scaling up the technology needs more research [104]. A close technique was used for encapsulation of antitubercular drugs separately [105] or in several combinations [106]. The study was aimed at evaluation of the chemotherapeutic potential of econazole encapsulated in alginate nanoparticles alone or in combination with other drugs against murine tuberculosis [106]. Chitosancoated nanoparticles were recovered by centrifugation after overnight incubation in the original solution [105-107]. They had an average size of $\sim 230 \mathrm{~nm}$ and a polydispersity index of 0.44 . Encapsulation efficiency was more than $70 \%$. Interestingly, the loading capacity was claimed as $480-730 \mathrm{mg}$ of drug per $100 \mathrm{mg}$ of alginate [105-107]. Oral treatment of mice infected with Mycobacterium tuberculosis $\mathrm{H} 37 \mathrm{Rv}$ with either free or encapsulated drugs resulted in total clearance of bacilli in lungs and spleen compared with untreated control, but the required dosing frequency was reduced by 15 -fold $[105,106]$. It was also valid if the nanoparticles were administered by nebulization to guinea pigs infected by the same bacilli. The aerodynamic characteristics of the droplets were as follows: $81 \%$ of them were in the size range 0.4 $2.1 \mu \mathrm{m}$; mass median aerodynamic diameter $1.1 \mu \mathrm{m}$; geometric standard deviation $1.7 \mu \mathrm{m}$. The nanoparticles did not display hepatotoxicity, as shown by the levels of serum bilirubin, alanine aminotransferase and alkaline phosphatase [107]. The doses were as follows: isoniazid $10 \mathrm{mg} / \mathrm{kg}$, rifampicin $12 \mathrm{mg} / \mathrm{kg}$ [105-107], pyrazinamide $25 \mathrm{mg} / \mathrm{kg}$ (orally) $[105,106]$ or $15 \mathrm{mg} / \mathrm{kg}$ (oral if free and nebulized if encapsulated) [107], ethambutol $16 \mathrm{mg} / \mathrm{kg}$ $[105,106]$, econazole $3.3 \mathrm{mg} / \mathrm{kg}$ body weight [106]. For a mouse weighting $20 \mathrm{~g}, 1.43 \mathrm{mg}$ of dried nanoparticles was a therapeutic dose. Free drugs were administered in $50 \mu \mathrm{l} 1: 4(\mathrm{v} / \mathrm{v})$ methanol: distilled water [105]. For a 400 -g guinea pig the dose was $21.3 \mathrm{mg}$ of dried nanoparticles. The drugs for nebulization were suspended in $4 \mathrm{ml}$ of physiological saline [107]. Free drugs remained at detectable level in tissues for 12-24 hours after a single administration. In contrast, encapsulated drugs remained at least for 10 days. Alginate nanoparticles can serve as an ideal carrier for the controlled release of antitubercular drugs [105-107], moreover, they are inhalable [107]. It should be noted, however, that other authors did not recommend chitosan-coated nanoparticles produced using the same technique for pulmonary drug delivery by nebulization because of their too big size $(556 \mathrm{~nm}$, polydispersity 0.29 ) and instability [108], although this technique has been reported to be able to generate much smaller nanoparticles, as discussed above. The nanoparticles were unstable in $10 \%$ fetal calf serum, simulated lung fluid and even in $0.9 \%$ saline, despite zeta potential of $29 \mathrm{mV}$. Human alveolar type 1 epithelial cells at 16000 cells $/ \mathrm{cm}^{2}$ incubated for 24 hours with medium containing these nanoparticles at $2000 \mu \mathrm{g} / \mathrm{ml}$ showed $\sim 80 \%$ viability, and their DNA content 
decreased to $76 \%$. At $100-1000 \mu \mathrm{g} / \mathrm{ml}$ significant dose-dependent cellular uptake of nanoparticles with encapsulated indocyanine green after 2 hours of incubation was shown. The nanoparticles released $27 \%$ of encapsulated bovine serum albumin in water through a dialysis bag within 2 days and more than $80 \%$ for 21 days. The goal of the study was to determine the most promising formulations for further development as carriers for pulmonary delivery of biological agents [108]. In the same way, alginate nanoparticles coated with quaternized chitosan were prepared. The reaction mixture was equilibrated overnight, and then the nanoparticles were separated by ultracentrifugation. Bovine serum albumin encapsulation efficiency increased with protein concentration up to $1 \mathrm{mg} / \mathrm{ml}$. Further increase promoted aggregation. The nanoparticles were spherical with a fluffy surface. Their zeta potential was $10 \mathrm{mV}$. Their size increased with the concentration of quaternized chitosan from 280.5 to $1152 \mathrm{~nm}$, and further increase led to aggregation. This concentration increase also promoted the encapsulation efficiency up to more than $40 \%$, while decreasing the loading efficiency from more than $15 \%$ to $\sim 10 \%$. Particle size, encapsulation efficiency and protein release rate in $\mathrm{HCl}$ (up to $22 \%$ for 100 hours) also increased with chitosan molecular weight. Increasing degree of substitution of chitosan did not influence the particle size, but increased the encapsulation efficiency and slowed down protein release, although burst release was still observed. Therefore, the release of the encapsulated protein can be tailored by choosing the molecular weight and degree of substitution of quaternized chitosan. The nanoparticles were intended for oral delivery of proteins [109]. A close technique was used for salbutamol sulfate encapsulation. The $\mathrm{pH}$ of the solutions of chitosan (despite initial preparation in $1 \% \mathrm{CH}_{3} \mathrm{COOH}$ ) and sodium alginate was adjusted to 5 . The characteristics of the particles were as follows: size $288 \mathrm{~nm}$, zeta potential $23 \mathrm{mV}$, encapsulation efficiency $21 \%$, stomach retention time 11 hours (in vitro). Less than $75 \%$ of the encapsulated drug was released to fasted state simulating gastric fluid for 12 hours, but fast release at the initial phase was observed. The size of the particles prepared without $\mathrm{pH}$ adjustment or when chitosan was added before $\mathrm{CaCl}_{2}$ was more than $800 \mathrm{~nm}$. In the terms of drug release profile or size this formulation excelled 24 others containing other polymers. The nanoparticles could be used for drug absorption enhancement [71]. For encapsulation of plasmid DNA encoding green fluorescent protein a different technique was used. The aim of the study was the development of several chitosan-containing nanoparticulate carriers of DNA and their comparison. In this case, the solutions of $\mathrm{CaCl}_{2}$, DNA and chitosan were dripped one after another from a syringe to that of sodium alginate containing dextran sulfate. After incubation in the reaction mixture under stirring the nanoparticles were separated by centrifugation. Their characteristics were as follows: average size $196 \mathrm{~nm}$, polydispersity index 0.15 , zeta potential $27 \mathrm{mV}$, encapsulation efficiency $95 \%$. The nanoparticles excelled those without dextran sulfate and those of pure chitosan in transfection efficiency of HEK 293 cells at $1 \mu \mathrm{g}$ of DNA for 48 hours. Unlike Lipofectamine, all the preparations of nanoparticles were not cytotoxic for these cells but had less tranfection efficiency. The nanoparticles could be used as gene carriers in vitro and in vivo due to their biocompatibility [110].
As can be seen, dripping method is very popular because of its conveniency. It does not require special skills and equipment, for pipette (or syringe) and stirrer (or ultrasonic bath) are enough. If the cells to be encapsulated are admixed to $\mathrm{CaCl}_{2}$ solution, they never come into contact with sodium alginate that may be toxic to animal cells [111]. Moreover, the modern dripping techniques allow to generate nanoparticles.

\section{Dripping sodium alginate into $\mathrm{CaCl}_{2}$}

Dropwise addition of sodium alginate solution to $\mathrm{CaCl}_{2}$ solution is also widely employed. In this case the drug being encapsulated is always admixed to sodium alginate solution, because this drop becomes a particle. Drop size and shape determine those of the particle.

Dropwise addition of sodium alginate solution containing cyclophosphamide to $\mathrm{CaCl}_{2}$ solution yielded beads having average diameter of $1 \mathrm{~mm}$. Bead drying retarded the release of $90-95 \%$ of the encapsulated drug into physiological saline from 80-100 minutes to 140-160 minutes. The beads could be suitable as a component of anticancer pharmaceutics because of their ability to be internalized by tumor cells [112]. Similarly produced microparticles with encapsulated crude turnip extract (applied in phosphate buffer) contained $0.295 \mathrm{mg}$ of protein per $1 \mathrm{ml}$ of matrix. Their average diameter was $390 \mu \mathrm{m}$, and the encapsulation efficiency was $70 \%$. They were used for treatment of wastewater containing phenol and chlorophenols, but the reaction products remained mainly in solution. The immobilization did not cause significant change of the enzyme catalytic constants. Polyethylene glycol protected the enzyme from inactivation, although some enzyme release to the solution still occurred. After each cycle the beads were washed to remove brown particles appearing inside them, and treated with $\mathrm{CaCl}_{2}$. This step was essential for maintaining enzyme activity. Optimization of cycle time allowed to extend bead reusability to 15 cycles. Encapsulated peroxidase displayed higher efficiency of phenol removal but lower thermal stability than covalently immobilized enzyme. Development of a large-scale economically feasible process for industrial effluent treatment was expected [113]. In the case of beads produced using the same technique but in the presence of $0.9 \% \mathrm{NaCl}$ strong swelling for the lowest molecular weight alginate tested was shown. Strong weight loss during bead preparation for the highest molecular weight alginates [2] and for those having low guluronic acid content and taken at low concentrations $[2,114]$ was observed. Without $\mathrm{NaCl}$ the weight loss in all cases was less than 4\% [114]. For the medium molecular weight alginate small weight loss was reported. The aim of the respective study [2] was the preparation of hydrogels with the applicability for immobilization of biological species. Indeed, cells can also be encapsulated using this technique. The pellet of yeast Candida tropicalis SSK01 cells was aseptically mixed into sodium alginate solution in Tris-acetate buffer $(\mathrm{pH}$ 7.5). The resulting solution was dripped into $\mathrm{CaCl}_{2}$ solution using a micropipette. After incubation under stirring the beads were washed with the same buffer and stored in it at $4{ }^{\circ} \mathrm{C}$. The applicability of the immobilized cells for phenol degradation was demonstrated. The most significant operating factors and optimum levels of phenol degradation were found by means of statistical optimization 
approach. Functional relationship between independent variables like initial phenol concentration, temperature, $\mathrm{pH}$ and maximum percentage of phenol degradation was found out [115]. Almost the same technique was used for coencapsulation of human bone marrow mesenchymal stem cells at $2 \cdot 10^{6}$ cells per $1 \mathrm{ml}$ of sodium alginate solution and an antibody to bone marrow protein 2 at $25 \mu \mathrm{g} / \mathrm{ml}$. Sodium alginate was coupled with RGD sequence (cell attachment peptide) and partially oxidized to increase its degradability. An in vitro experiment showed that the beads (average diameter $1 \mathrm{~mm}$ ) released the encapsulated antibody in phosphate buffered saline for 10 days but still retained more than $30 \%$ of it after 14 days. The images acquired by means of bright field microscopy revealed uniform cell distribution inside the beads. The beads were transplanted into critical size calvarial defects in immunocompromised mice. The antibodies captured endogenous bone morphogenic protein 2 and presented it to the stem cells, promoting their osteogenic differentiation and therefore bone repair and regeneration. The bone had a lamellar pattern, contained viable osteocytes, and was rimmed with osteoblasts participating in its formation. Dystrophic calcifications were not observed. Osteocytes and osteoblasts were observed even if the beads contained only the antibody or only the stem cells. But if the antibody was nonspecific and there were no encapsulated stem cells (i.e. negative control), fibrous and vascular connective tissue components consisting of interwoven bundles of collagenous fibers and calcium alginate were observed instead of regenerated bone. The bone regeneration strategy is a promising modality for repair and regeneration of craniofacial, axial and appendicular bone defects [116]. The co-immobilization of microalga Chlorella vulgaris with auxin-producing bacterium Azospirillum brasilense or with chemically synthesized indole-3-acetic acid in calcium alginate beads by the same technique promoted microalga multiplication and chlorophyll a, chlorophyll b, $\beta$-carotene, lutein and violoxanthin production. During solidification of the alginate into beads, numerous internal cavities of various sizes are formed randomly, and they provide the microenvironment for cells. Increasing biomass production of immobilized microalgae has important implications for environmental cleanup and some industrial processes [117]. The promoting effect of alginate oligosaccharides on root formation and growth in rice was also mediated by endogenous indole-3-acetic acid [30]. Using the same technique coupled with chitosan coating and fluid bed drying, beads having irregular structure and size of about $1 \mathrm{~mm}$ with encapsulated Bifidobacterium breve were prepared. After initial binding to alginate, the penetration of chitosan into the gel was seen only after 1080 min without any changes before this time point. Swelling and disintegration of the beads were $\mathrm{pH}$-dependent. They released encapsulated bacteria into simulated intestinal solution (by bead disintegration) for $120 \mathrm{~min}$ after incubation in simulated gastric solution for 1 hour. In contrast to this, the beads without chitosan coating began to release bacteria at the stage of incubation in gastric solution, therefore they released much lower amount of viable cells to the simulated intestinal solution. The beads were produced in a scalable matter as a uniform granular product that could be used as a dry enteric delivery formulation for probiotic bacteria [118]. A close technique was used for the immobilization of bacteria. The main difference was the use of $\mathrm{CaCl}_{2}$ solution in milk. After 8-10 hours of milk fermentation the concentration of the released bacteria was less than $10^{8} \mathrm{cfu} / \mathrm{ml}$. (The ratio of beads to milk was adjusted to $\sim 17 \%$ ). Lactobacillus helveticus and Streptococcus thermophilus grew in the beads, while Lactobacillus delbrueckii ssp. bulgaricus did not [43]. Rifampicin and fluconazole were separately encapsulated into chitosan-coated alginate beads with a diameter of $1 \mathrm{~mm}$ in a similar way. Air drying retarded drug release into physiological saline. Further drug release rate decrease by $30 \%$ for up to 12 hours was observed with the increase of chitosan shell thickness from $25 \mu \mathrm{m}$ to $125 \mu \mathrm{m}$ that was, in turn, determined by the employed chitosan concentration. The use of these beads as antitubercular medicaments with prolonged action was suggested [119]. A slightly different technique was employed for the encapsulation of sunflower oil extract from tunic of ascidia. Sonicated emulsion of this extract in aqueous sodium alginate solution containing Tween 80 and activated charcoal powder at 50 ${ }^{\circ} \mathrm{C}$ was dripped into $\mathrm{CaCl}_{2}$ solution through a syringe nozzle. The resulting beads were incubated in this solution followed by separation, incomplete drying, and transfer into chitosan solution. The characteristics of the beads prepared using the nozzles having diameter of 0.2 and $0.7 \mathrm{~mm}$ were as follows: irregular shape, smooth surface, size 1.4 and $2.2 \mathrm{~mm}$, bead mass 2.5 and $3.1 \mathrm{mg}$, carotenoid content 0.9 and $0.12 \mu \mathrm{g} / \mathrm{bead}$, respectively. They were stable in pure water, ethanol, and in $\mathrm{HCl}$ at $\mathrm{pH} 2.5$ for at least 240 hours. Storage stability of dry beads for 5 months was shown. No visible degradation in water at $20-100{ }^{\circ} \mathrm{C}$ for $20 \mathrm{~min}$ was observed. The degradation rate increased with $\mathrm{pH}$; however, the $\mathrm{pH}$ increase from 9.2 to 11 had no effect. The beads were suggested as a component of functional, i.e. healthy food products [120]. In another report multilayer coating was employed. Sodium alginate containing bovine serum albumin was extruded into stirred $\mathrm{CaCl}_{2}$ solution through a commercially available encapsulator nozzle. The particles were than covered with poly-L-lysine, pectin, again with poly-Llysine and finally with a new sodium alginate layer. Their diameter was $\sim 400 \mu \mathrm{m}$ and depended on the nozzle used. The microparticles were stable during incubation in simulated gastric fluid for 3 hours followed by incubation in simulated intestinal fluid for up to 24 hours. They were stable for 24 hours at pH 1-9 as well, and had good mechanical stability. All the tests above were carried out using empty microparticles. The loaded ones released half of the protein amount compared with the microcapsules without pectin and second poly-L-lysine layers. The microcapsules are expected to allow safe and efficient oral delivery of live bacterial cells for various clinical applications [121]. The encapsulator without subsequent coating was also used in the case of $\alpha \mathrm{TC} \Delta \mathrm{PC} 2$ cells. These cells synthesize prohormone convertase 1/3-derived proglucagon products. Injection of $1.5-2 \mathrm{ml}$ of the microcapsules with a size of $500-700 \mu \mathrm{m}$ in a total volume less than $3 \mathrm{ml}$ to the intraperitoneal cavity improved glucose handling in mouse models of type 2 diabetes, providing a model for evaluating long-term delivery of proglucagon-derived peptides via cell therapy. Each mouse received about $4 \cdot 10^{7}$ encapsulated cells. In the case of mice with genetic defects in the leptin receptor signaling pathway the animals had significantly higher body temperature during acute housing at $4^{\circ} \mathrm{C}$. If left untreated, these mice have defective cold-induced 
thermogenesis, and their glucose-stimulated insulin secretion was also defective. The treatment did not change epididymal white adipose tissue and intrascapular brown adipose tissue of these defective mice [122]. Alginate microparticles coated with oligochitosan and containing encapsulated MCF-7 breast cancer cells were converted to alginate-chitosan microcapsules by treatment with EDTA dissolving the calcium alginate core. Sodium alginate solution containing the cells was dripped into $\mathrm{CaCl}_{2}$ solution by means of a device with coaxial air flow or using an electrostatic generator of microparticles. The possibility of producing microcapsules with predetermined size by the means of the latter method was underlined but not demonstrated. Physiological saline was used for washing between the incubation steps. The microcapsules produced by two methods had average size of $750 \mu \mathrm{m}$ and $300 \mu \mathrm{m}$, respectively, and were prone to swelling. Chitosan membrane thickness was about $82-89 \mu \mathrm{m}$. Tumor spheroids were generated inside the microcapsules. The cells in the spheroids can better mimic cell behavior in a small size solid tumor than the cells in a monolayer culture. This new in vitro model of anticancer drug screening has advantages of spheroids produced using classic techniques [123]. In another report, sodium alginate solution containing drug (salbutamol sulfate), guar gum and/or pectin was dropped through a syringe into $\mathrm{CaCl}_{2}$ solution and incubated under stirring. The beads formed were separated by filtration, washed and dried. Their mean diameter was $0.7-2.1 \mathrm{~mm}$ depending on concentration of polymers. Encapsulation efficiency was $65-91 \%$. The encapsulated drug was completely released during 3-7 hours of incubation in simulated intestinal fluid following 2 hours in simulated gastric fluid, where less than $20 \%$ was released. Sodium alginate concentration increase made the drug release more sustained. Guar gum and pectin improved it further, and the best formulation contained both polymers. The beads are promising pharmaceutical dosage forms, and better patient compliance can be expected [124]. In the same salbutamol sulfate encapsulation technique $\mathrm{BaCl}_{2}, \mathrm{Al}_{2}\left(\mathrm{SO}_{4}\right)_{3}$ and magnesium aluminum silicate could be used instead of $\mathrm{CaCl}_{2}$. Encapsulation efficiency was more than $77 \%$. The size of oven dried nanoparticles produced using $2 \%$ sodium alginate and the respective salts was 921, 863, 899 and $782 \mathrm{~nm}$, and increased with sodium alginate concentration. $90 \%$ or more of the encapsulated drug was gradually released into phosphate buffer for 9 hours. Air-dried nanoparticles were larger in size due to incomplete dehydration and showed slower drug release, but the encapsulation efficiency was comparable with that of oven dried ones. Particle characteristics did not change after 3 months of storage at $40^{\circ} \mathrm{C}$. Nanoparticles are expected to spread out in the gastrointestinal tract thus improving drug absorption and reducing local irritation [88]. Dried beads with encapsulated salbutamol sulfate $\left(\mathrm{CaCl}_{2}\right.$ was used) were dispersed in Eudragit S-100 solution in acetone for coating. The dispersion was poured into liquid paraffin, incubated under stirring to evaporate acetone, and finally the beads were washed with hexane and dried. They were spherical with a smooth surface, their size was $640-690 \mu \mathrm{m}$ with narrow size range, and encapsulation efficiency was 68-86\%. It decreased with crosslinking time increase because of drug leakage and increased with sodium alginate and $\mathrm{CaCl}_{2}$ concentration. As these concentrations and coating level increased, drug release rate decreased. Coating prevented drug release at $\mathrm{pH} 1.2(\mathrm{HCl})$ and 6.8 (phosphate buffer). At $\mathrm{pH} 7.4$ (phosphate buffer) more than $70 \%$ of the encapsulated drug was released for 21 hour. The preparation was intended for chronotherapeutic drug delivery for nocturnal asthma treatment and claimed as colon specific [125]. The same technique was used to produce microspheres with a diameter $\sim 700 \mu \mathrm{m}$ containing encapsulated bovine serum albumin conjugated with tetramethyl rhodamine. They were incubated in $\mathrm{CaCl}_{2}$ solution for 72 hours, then removed by filtration, washed and resuspended in $70 \%$ ethanol. After that they were shaken in silk fibroin solution, ultrasonicated to break down the aggregates and shaken again. They were washed with either water or methanol, dried and coated again. There were 3 fibroin layers in total. The shell was $\sim 10 \mu \mathrm{m}$ thick. Loading efficiency was $\sim 15 \%$ and was not changed by the coating. The coating retarded the conjugate release in phosphate buffered saline from 10 to more than 16 days. Incubation in EDTA solution led to alginate core dissolution, but the fibroin shell remained. Coating with fibroin has potential for biosensor and diverse array of controlled drug delivery applications [126].

For large-scale production of alginate beads having diameter of several millimeters a special device has been proposed. In this device, $\mathrm{CaCl}_{2}$ solution is consistently fed to the center of the rotating disk, and above the disk there is a bowl fed with sodium alginate solution. The bowl is also rotating and spraying alginate onto the rotating disk where it contacts calcium chloride. The average bead diameter is determined by the disk diameter, its angular velocity, alginate solution viscosity and feed rate. It should be noted that alginate solution sterilization causes its depolymerization thus decreasing viscosity. From the 'parent' beads smaller 'satellite' beads are being detached making the bead size distribution bimodal. Improper selection of the parameters may result in producing undesirable alginate filaments instead of beads. The system could be applied to the immobilization of microorganisms, as well as plant or animal cell cultures. The device can be inserted in a fermentor for the immediate use of the beads. If the fermentation medium contains calcium, it can be used for bead production instead of $\mathrm{CaCl}_{2}$ solution [127].

This variant of dripping method is also very popular and convenient. However, the method is generally limited to beads and microparticles because of difficulties with generating small alginate droplets due to its high viscosity.

\section{Calcium alginate nanoparticles as stabilizing agents for other nanoparticles}

The use of calcium alginate particles is not limited to encapsulation of various substances in molecular form. They can also be used as carriers for nanoparticles. The nanoparticles can be synthesized either in advance, as discussed above for the case of gold nanoparticles [89], or simultaneously with alginate particles, as described below. The synthesis of alginate particles using other ones as cores is not included here because it has been analyzed separately (see above).

Dropwise addition of sodium alginate solution containing nifedipine to $\mathrm{CaCl}_{2}$ solution in 1:1 alcohol/water mixture followed 
by chitosan solution addition led to spherical nanoparticles having size about $20-50 \mathrm{~nm}$ and fluffy surface with nifedipine nanocrystals inside. The average drug content was $6.7 \mu \mathrm{g} / \mathrm{mg}$, but less than $70 \%$ of the encapsulated nifedipine could be released. The particles could release nifedipine for more than 10 hours in a $\mathrm{pH}$-sensitive way with initial burst release for 2 hours. Although two of the three release media used were phosphate buffers, diffusion controlled release was revealed. This nanosystem offers an interesting potential for the delivery of hydrophobic compounds. If patients take it before sleep, drug concentration in plasma can reach peak value for initial burst, and then constant therapeutic concentration may be maintained by continuous and controlled release [128]. In another report dealing with nanoformulation of curcumin for delivery to cancer cells, ethanolic curcumin solution was added to $\mathrm{CaCl}_{2}$ solution in water. Under these conditions some fraction of curcumin formed nanocrystals, and excess curcumin was removed by centrifugation. If $\mathrm{CaCl}_{2}$ solution contained Pluronic F127, no precipitation of free curcumin was observed. The resulting solution was added dropwise to sodium alginate solution under stirring, followed by dropwise chitosan addition, and spherical particles with solid dense structure, uniform size distribution and a mean size of $100 \mathrm{~nm}$ were separated by centrifugation after incubation in the original solution. The $\mathrm{pH}$ of the final solution was made 4.7 by adjusting the values of alginate and chitosan solutions. Encapsulation efficiency in the presence of pluronic was more than $10 \%$, and about $75 \%$ of encapsulated curcumin was released from lyophilized nanoparticles for 72 hours in phosphate buffered saline. Comparable internalization by HeLa cells was observed for free and encapsulated curcumin at $2 \mu \mathrm{M}$. The inhibition of cell proliferation at $2-12 \mu \mathrm{M}$ was comparable as well. Half-maximal inhibitory concentration values were 13.28 and $14.34 \mu \mathrm{M}$, respectively. The empty particles were nontoxic for those cells at 50-500 $\mu \mathrm{g} / \mathrm{ml}$. The latter concentration was found to be optimal, and at higher concentrations (up to $1000 \mu \mathrm{g} / \mathrm{ml}$ ) small decrease in cell viability was observed. The nanoparticles have the potential to meet some of the challenges of nanoformulation of hydrophobic drugs for delivery to cancer cells and were claimed as holding immense potential in delivery of hydrophobic drugs in general [129].

The use of calcium alginate particles as shells to other particles looks very promising. They are much less prone to dissolution than naked drug nanoparticles. Moreover, alginate particles can be functionalized and targeted. Obviously, the diffusion coefficients of nanoparticles are much smaller than those of molecules. Therefore, it is preferable to encapsulate drug nanoparticles instead of free drugs, provided this possibility exists.

\section{Comparison of external and internal gelation}

A comparison of external and internal gelation techniques showed that the latter ones yielded more porous beads [114], although it might be explained by $\mathrm{CO}_{2}$ liberation from $\mathrm{CaCO}_{3}[130]$ in the case of internal gelation. The bead shrinkage during gelation was higher in the case of external gelation, but in simulated gastric juice both types of beads shrunk similarly. Their reswelling at pH 7 was almost similar as well. Internal gelation led to calcium concentration gradient only near the gel surface. It did not depend on alginate concentration, and did not exist throughout the gel. External gelation led to steep gradient increasing with alginate concentration, and there was also alginate concentration gradient up to hollow cores. In the case of DNA encapsulation its gradient was observed as well. The encapsulation yield was $97 \%$ and $80 \%$, respectively, independently of alginate composition. Externally gelled beads adsorbed 2 times more DNAse [114]. For their synthesis sodium alginate solution containing DNA was extruded through a syringe tip needle apparatus into $\mathrm{CaCl}_{2}$ solution. Bead diameter was in the millimeter range. In the case of internal gelation, sodium alginate solution containing DNA and sonicated $\mathrm{CaCO}_{3}$ powder was extruded into canola oil containing acetic acid. After the reaction the beads were washed with $\mathrm{CaCl}_{2}$ solution containing Tween 80 [114,131]. Ca: Na molar exchange ratios at initial and equilibrium time were 0.5 and 0.6 , respectively [114]. Chitosan and poly-L-lysine uptake by the beads during coating decreased with polymer molecular weight increase. The coated beads were stored in $\mathrm{CaCl}_{2}$ solution. Decreasing alginate guluronic acid content and increasing coating time, poly-L-lysine (only for external gelation) or chitosan molecular weight and degree of deacetylation provided better protection of DNA from DNAse irrespective of bead forming techniques, however, no double-strand DNA was found after 40 minutes of incubation with the enzyme. In the case of uncoated and poly-L-lysine-coated internally gelled beads lyophilization with subsequent rehydration strongly improved the protective properties [128]. The study aimed at protection of immobilized DNA from nuclease attack in order to use it as a trap to monitor levels of food related carcinogens in vivo. The recovery and purification of the DNA and its adducts is necessary for molecular biological assay to determine the level and nature of adduct formation and the extent of DNA damage [114,131].

However, in another report it was shown that the beads produced by internal gelation had lower mean sizes $(2.2 \mathrm{~mm}$ at $\mathrm{pH} 4$ and $3 \mathrm{~mm}$, respectively), higher shrinking $(0.88 \%$ at $\mathrm{pH} 4$ and $0.6 \%$, respectively) and more homogenous structure than those produced by external gelation. The shrinking increased with gelation $\mathrm{pH}$ decrease. The beads were produced by dripping alginate- $\mathrm{CaCO}_{3}$ mixture in canola oil containing Span 80 into acetate buffer (for internal gelation) or into $\mathrm{CaCl}_{2}$ solution (for external gelation). The internal $\mathrm{pH}$ of the bead was higher than that in the gelling solution. The empty beads were suspended in blue dextran solution and transferred to water. Blue dextran release for 15 days was $60 \%$ and $11 \%$, respectively [68].

\section{Aerosolization method}

In aerosolization method, the solution of sodium alginate is converted to aerosol by a special device called nebulizer or atomizer (Figure 5). These terms are synonymous. In such devices pressurized carrier gas is needed for aerosol formation. Correspondingly, aerosolization can be called nebulization or atomization. The aerosol formed is carried by a flow of the carrier gas into $\mathrm{CaCl}_{2}$ solution. The aerosol droplets become enclosed in gas bubbles inside $\mathrm{CaCl}_{2}$ solution, therefore gas flow must not be too high in order to provide them enough time to diffuse to the solution. The method resembles dripping because aerosol also consists of 
drops. However, here the only possibility is to aerosolize sodium alginate solution into $\mathrm{CaCl}_{2}$ solution because in the reverse case bulk plain hydrogel is formed [2]. Another significant difference is nozzle placement. In dripping method, the nozzle is usually placed above $\mathrm{CaCl}_{2}$ solution for drop formation. In aerosolization method the nozzle is usually placed inside $\mathrm{CaCl}_{2}$ solution, because in the opposite case the yield of particles is very low. The carrier gas is usually air supplied by a pump. The process is accompanied by formation of secondary aerosol having other drop size distribution. Secondary aerosol leads to loss of solution and therefore to lower yields, but it can be prevented using a vigreux [63]. Aerosolization is usually considered as a method limited to production of alginate microparticles [77]. However, it has a potential of generating nanoparticles as well $[63,132,133]$ because the aerosol drops can be made very small up to nanosize. It should be underlined that aerosolization is usually time-consuming, therefore, a fraction of nanoparticles stays in $\mathrm{CaCl}_{2}$ solution for a prolonged time while other nanoparticles are being formed. If the concentration of $\mathrm{CaCl}_{2}$ is too high, the nanoparticles will aggregate and even deposit [63]. It also limits the possibilities of scaling up the technique without changing the $\mathrm{CaCl}_{2}$ solution.

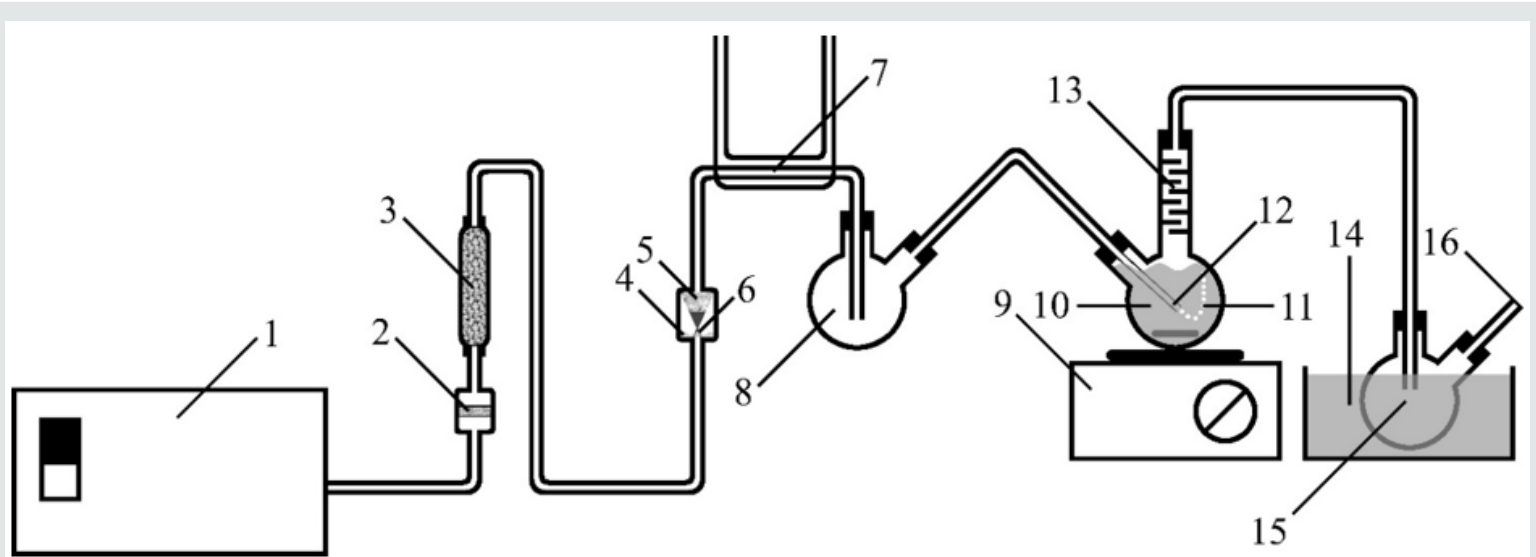

Figure 5: Aerosolization method. 1 - air pump; 2 - air filter (optional); 3 - air dryer (optional); 4 - sodium alginate solution containing drug; 5 - aerosol; 6 - nozzle; 7 - heater (optional); 8 - aerodynamic classifier (optional); 9 - magnetic stirrer (optional); 10 - $\mathrm{CaCl}_{2}$ solution; 11 - air bubbles; 12 - capillary; 13 - vigreux (optional); 14 - liquid nitrogen; 15 - cold trap; 16 - exhaust tube. The items 14 to 16 are used only with optional vacuum pump.

The process of liquid atomization by a co-flowing gas stream is rather complex because of its multistage nature (Figure 6). At first, instabilities develop in the liquid layer, causing the formation, growth and detachment of liquid tongues. The initially formed large non-spherical parcels continuously break up into droplets. At a certain distance the break-up process finishes, and then the droplet size increases slowly due to coalescence [134]. The description above refers to the most common nebulizer type. There are, however, other types as well, those with hydraulic/airless spray nozzles being an example. The hydraulic nozzles utilize the hydraulic pressures to disintegrate the liquid jet into fine droplets [135].

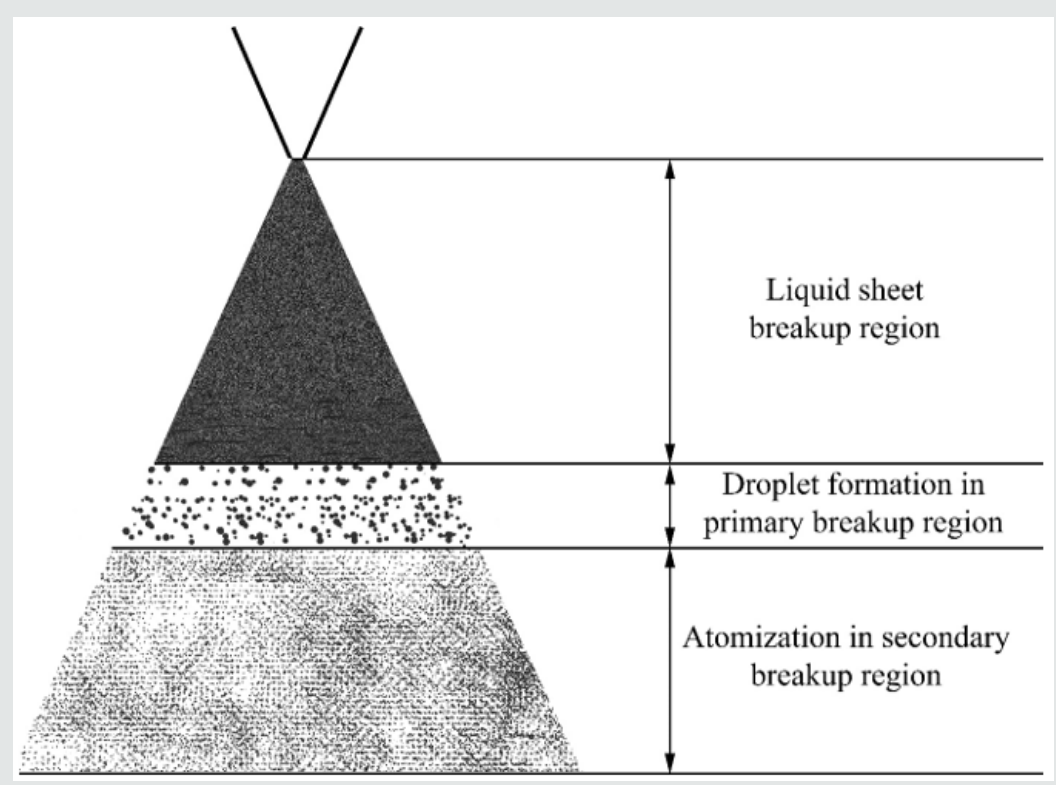

Figure 6: Atomization regions of the spray from the hollow cone nozzle [136]. 
In the simplest variant of the aerosolization method, sodium alginate solution containing horseradish peroxidase and a small amount of sodium phosphate buffer was aerosolized above slowly stirred $\mathrm{CaCl}_{2}$ solution using a manual disperser. After 2 hours of incubation the beads were separated by filtration and washed with phosphate buffer solution. The droplets produced by the disperser had rather big size, therefore the size of the beads was about $0.5 \mathrm{~mm}$. The beads contained $1.18 \mu \mathrm{g}$ of enzyme per $1 \mathrm{mg}$ of dry bead mass, and the encapsulation yield was $85 \%$. The activity of immobilized horseradish peroxidase was significantly higher than that of the free enzyme. The immobilization also prevented enzyme activity loss at $60^{\circ} \mathrm{C}$ for 1 hour, and the activity of the encapsulated enzyme was still measurable after storage of the beads for 7 months in phosphate buffer solution at $4{ }^{\circ} \mathrm{C}$ [137]. This was not the only example of enzyme activation after its encapsulation in calcium alginate. It was shown that at $\mathrm{pH}$ 5.5-6 or in alkaline medium the activity of horseradish peroxidase encapsulated in calcium alginate beads was higher than that of free enzyme. The reaction studied was phenol oxidation, and the beads were claimed as promising for continuous phenol removal from wastewater [41]. The encapsulation technique used was dropping $\mathrm{CaCl}_{2}$ solution containing dextran and the enzyme into rapidly stirred sodium alginate solution followed by capsule transfer to a new $\mathrm{CaCl}_{2}$ solution. The technique was also used for the encapsulation of monkey kidney cells, and their growing inside the capsules was observed. Capsule membrane thickness was found to be dependent on incubation time in sodium alginate solution, the capsule size and the concentrations of sodium alginate and $\mathrm{CaCl}_{2}$. The possible fields of application of cell encapsulation techniques include bioreactors, artificial organs and others [111]. Enzyme activation caused by immobilization in alginate is not limited to external gelation techniques; it was also observed for glucose oxidase immobilized using emulsification-internal gelation technique [67], as discussed above.

For pre-industrial scale production of alginate microparticles an aerosolization device has been suggested. Compressed air after drying and filtration is directed to a piston pump fed with sodium alginate solution containing hydroxypropyl methyl cellulose and lysozyme being encapsulated. The solution is directed to airless spray-gun. The atomized droplets are collected into a tank filled with $\mathrm{CaCl}_{2}$ solution (containing chitosan and acetic acid) and placed on a rotating platform. The particles are collected by filtration, washed and dried. Volume mean diameter of the produced particles depended on experimental conditions (sodium alginate concentration, nozzle size, swelling in water or phosphate buffered saline) and could be in the range from 69 to $274 \mu \mathrm{m}$ (without precise control). The particles larger than $400 \mu \mathrm{m}$ were also present. All the particles seemed to be aggregates. The particles released $40-90 \%$ of encapsulated lysozyme during the incubation in glycine buffer for 8 hours and then in phosphate buffered saline for 16 hours. The device appears to be suitable for production of microparticles showing gastro-resistance and suitability for oral applications or animal husbandry by processing feeding solutions of sodium alginate in a wide range of viscosities [138].
Aerosolization of sodium alginate solution containing drug diltiazem hydrochloride into $\mathrm{CaCl}_{2}$ solution led to microparticles. In order to prevent their agglomeration, $20 \%(\mathrm{v} / \mathrm{v})$ ethanol was added to $\mathrm{CaCl}_{2}$ solution. The microparticles formed were dried and than rehydrated. Air flow rate increase decreased their size and influenced their polydispersity. The buffers in the $\mathrm{CaCl}_{2}$ and rehydration solutions (because of possible solubilization of alginate) and the presence of chitosan in the $\mathrm{CaCl}_{2}$ solution were other factors influencing particle size. The particles having various sizes from 200 to $1100 \mu \mathrm{m}$ were produced. The drug release profiles were influenced by $\mathrm{pH}$ of the release medium, molecular weight of chitosan and amount of the drug loaded into the particles, but the equilibrium concentration was always reached after $30 \mathrm{~min}$ because of the high water solubility of diltiazem hydrochloride. Drug load, in turn, could be increased by addition of $\mathrm{NaCl}$ to sodium alginate solution because it decreased the electrostatic interaction between the drug and alginate. The surface tension, viscosity and $\mathrm{pH}$ of the $\mathrm{CaCl}_{2}$ solution were identified as important factors for controlling particle agglomeration and stability as well as drug encapsulation [139].

The experiment with diffusion of 4 and $70 \mathrm{kDa}$ FITC-dextran from calcium alginate microparticles having sizes 20 and 841 (or 948) $\mu \mathrm{m}$ revealed that for the larger particles there was a lower number of crosslinks per surface area. For 500 kDa FITC-dextran the particles were impermeable. The particles were produced by aerosolization method and by inkjet printing. The inkjet engine fires sodium alginate solution through a print head into $\mathrm{CaCl}_{2}$ solution. The particles were used as artificial cells in the study of modeling diffusivity [140].

By aerosolization of alginate solution containing cells (islets isolated from mouse [141], rat or human [142] pancreas) and human serum albumin used as a stabilizer [141] into $\mathrm{BaCl}_{2}$ solution [141,142] containing histidine as buffer and $\mathrm{NaCl}$ [139] using an air-driven droplet generator microparticles were produced. Their diameter was $900-1100 \mu \mathrm{m}$ [141] or $700-800 \mu \mathrm{m}$ [142]. After their transplantation diabetic mice became normoglycemic, but hyperglycemia immediately reappeared after explantation of the microparticles. No rejection was observed [141,142]. Each mouse received 900-1000 [141] or 1800 [142] microencapsulated islet equivalents. The peak C-peptide response in these mice was delayed compared with that of healthy mice. No overgrowth of the explanted microparticles was observed. Cytokines were demonstrated to damage encapsulated islet cells in vitro, but there was no cellular reaction [141] or weak cellular reaction at the particle surface. Slight reduction of insulin secretion capacity of rat islets occurred after transplantation [142]. The use of $\mathrm{BaCl}_{2}$ as crosslinker provoked less fibrotic reaction than did $\mathrm{CaCl}_{2}[141]$. The same technique was used for porcine neonatal pancreatic cell cluster encapsulation, and the same positive effect was shown after their transplantation through a small abdominal incision into the peritoneal cavity of anesthetized diabetic mice. Each mouse received 10000 islet equivalents contained in $2.25 \mathrm{ml}$ of the microparticles. Cell differentiation occurred within the particles. Most capsules were free of overgrowth 2 and 20 weeks after 
transplantation, although at 6 weeks $43 \%$ of the capsules were overgrown [143]. The proliferaton index of the $\beta$-cells before and 6 weeks after transplantation was almost the same, but it dramatically decreased at 20 weeks. IgG was deposited on the cells inside the microparticles, i.e. it could diffuse through the capsule, but without causing adverse effects. Another problem was batch-to-batch variation of encapsulated cells [143]. It should be noted, however, that the human clinical trial with barium alginate microparticles having diameter in the range $255-750 \mu \mathrm{m}$ with median average diameter $340 \mu \mathrm{m}$ failed because of necrosis of all the transplanted encapsulated islets of cadaver origin. The capsules were found intact and surrounded by fibrous tissue containing thin-walled capillaries with a mild histiocytic response. Each person received 98200-227900 islet equivalents. The median number was 178200 . One person received three islet infusions for the 1st month and one more 6 months later. Another recipient received two infusions 10 months apart. The other two patients received one infusion each. Cytotoxic antibodies were detected in two persons. The loss of the encapsulated cells might occur due to either ischemic necrosis or inflammation [144]. The respective studies were aimed at the development of so-called bioartificial pancreas, i.e. pancreatic islets enclosed within immunobarrier membranes intended for transplantation to patients with type 1 diabetes [138-144]. In another report, Lactobacillus rhamnosus cell suspension in sodium alginate solution was atomized into $\mathrm{CaCl}_{2}$ solution followed by additional incubation in this solution. The resulting microparticles were recovered by sedimentation, incubated in chitosan solution under shaking, washed with $\mathrm{CaCl}_{2}$ solution and again recovered by sedimentation. They had size of $\sim 390-450 \mu \mathrm{m}$, loading efficiency of $38 \%$ and were roughly spherical in shape. The possibility of formation of biofilm-like globules inside the particles was shown. Such particles possessed higher resistance (in the terms of cell viability) to freeze-drying and to heat exposure at intermediate temperatures compared to those with encapsulated single cells. However, the resistance to refrigerated storage, simulated gastric juice and high temperatures was similar. Alginate particles were found to be overall superior to carrageenan ones. Chitosan-coated alginate capsules were characterized as the most suitable delivery system for the biofilm-like probiotics. Cell encapsulation represents the more plausible and widely accepted approach than development of heat-resistant probiotic strains via genetic modifications [145].

Aerosolization of sodium alginate solution containing mesoporous silica nanoparticles with a diameter around 500nm above calcium-containing solution using an air-dynamical nebulizer led to microspheres with a diameter around $20-30 \mu \mathrm{m}$ and silica nanoparticles inside. If the nanoparticles contained rhodamine $6 \mathrm{G}$, its release from microparticles in phosphate buffer increased with buffer concentration increase (maybe because of alginate solubilization, as discussed below) and decreased with alginate concentration increase, but only up to $1 \%$ alginate. Sustained release could be maintained for 40 days. Doxorubicin encapsulation into the nanoparticles suspended in MEM medium enhanced the efficiency of its delivery into cultured HepG2 cells for 4 hours about 1.85-fold. Drug concentrations used for the experiment were 1, 5 and $10 \mu \mathrm{M}$. Alginate functionalization with RGD-based targeting peptide using carbodiimide chemistry prior to particle synthesis enhanced drug delivery further, viz. about 1.6-fold, as shown for the case of fluorescein isothiocyanate. Functionalized microparticles specifically targeted $\alpha_{v} \beta_{3}$ receptor of HepG2 cells and were taken up by the receptor-mediated endocytosis pathway. At 2-10mg/ $\mathrm{ml}$ the microparticles without encapsulated doxorubicin were not cytotoxic, as revealed by incubation at $37{ }^{\circ} \mathrm{C}$ for 24 hours. The microparticles show significant potential as effective and visually observable transmembrane delivery carriers with high biocompatibility and targeting features for the intracellular controlled release of cell membrane-impermeable drugs. Further development of this nanomaterial could lead to a new generation of nanodevices for biomedical applications [146].

\section{The synthesis of calcium alginate particles with predefined size}

The size of nanoparticles produced by aerosolization of sodium alginate solution containing oligoguluronate (prepared by acid hydrolysis of alginate) and superparamagnetic iron oxide nanoparticles into $\mathrm{CaCl}_{2}$ solution under mild stirring was from 811 to $941 \mathrm{~nm}$ with wide size distribution. The nanoparticles showed a spherical morphology, whereas relatively rough surface was shown when the polyethylene glycol-chitosan graft copolymer was present in the $\mathrm{CaCl}_{2}$ solution. It should be noted that in the presence of the polymer the resulting $\mathrm{CaCl}_{2}$ concentration was 2 times less. The presence of the polymer as well as alginate relative concentration (in alginate-oligoguluronate mixture) influenced the swelling values. The parameters suggested to influence the particle size but not tested were atomizer output particle size, drying/conditioning of aerosol prior to crosslinking, concentration of reaction components, and reaction temperatures. The device used for the nanoparticle fabrication included a drying system and aerodynamic aerosol classifiers before and an empty collector after $\mathrm{CaCl}_{2}$ solution. This collector was assembled to the vacuum system. The nanoparticles were initially produced swollen, then freeze-dried, washed with water and then freeze-dried again. These magnetically responsive 'smart' nanoparticles showed promising characteristics for various drug delivery and biomedical applications [132]. Another experiment using the same device but carried out without oligoguluronate and iron oxide nanoparticles showed alginate concentration, air pressure, crosslinker type and air pressure taken together to be the main factors modifying particle size. The listing order here corresponds to the importance of these factors. In the case of swelling behavior in water only alginate concentration was a fundamental factor. Swelling could increase particle size more than four times. The mean size of the particles developed using two different nebulizers was 842 and $886 \mathrm{~nm}$ with a standard deviation of $7-12 \%$. The particles presented, in general, spherical shapes with highly rough surfaces. The yield was between $30 \%$ and $90 \%$. The technique is valuable for the preparation of particles for various applications, particularly for drug delivery purposes [133].

The size of calcium alginate nanoparticles produced by aerosolization of sodium alginate solution into $\mathrm{CaCl}_{2}$ solution may depend on the characteristics of gas bubbles formed in the latter solution, but we could not predict the size of the resulting 
nanoparticles [63]. Porosity and mean pore diameter of calcium alginate beads as well as bead size (2.2mm for control beads) were shown to increase with the concentration of carbon dioxide-forming agent $\left(\mathrm{CaCO}_{3}\right.$ or $\left.\mathrm{NaHCO}_{3}\right)$ incorporated into hydroxypropylmethylcellulose-containing sodium alginate solution being dripped into $\mathrm{CaCl}_{2}$ solution containing acetic acid. In the case of $\mathrm{NaHCO}_{3}$ pore size reached $39.7 \mu \mathrm{m}$ exceeding that of control $(0.4 \mu \mathrm{m})$ or that resulting from the use of $\mathrm{CaCO}_{3}(6.1 \mu \mathrm{m})$. At $\mathrm{NaHCO}_{3}$ : alginate ratio of $1: 1$ the released $\mathrm{CO}_{2}$ burst the beads, thus making them nonspherical. Despite the increased porosity and pore size, the release of encapsulated riboflavin to phosphate buffered saline became more prolonged (up to $\sim 2$ hours), and the bead surface became smoother with the increasing concentration of $\mathrm{CaCO}_{3}$ because of the participation of the released $\mathrm{Ca}^{2+}$ ions in alginate gelation. In the case of $\mathrm{NaHCO}_{3}$ the opposite effect on the drug release was observed. The presence of $\mathrm{CO}_{2}$ also improved bead buoyancy. $\mathrm{CaCO}_{3}$-containing beads are an excellent candidate for floating drug dosage systems. These systems remain buoyant in the stomach without affecting the gastric emptying rate for a prolonged period [130]. In contrast, our experiments displayed nanoparticle size reduction in the case of aerosolization of sodium alginate solution containing $\mathrm{NH}_{4} \mathrm{HCO}_{3}$ into $\mathrm{CaCl}_{2}$ solution containing acetic acid compared to the experiment without $\mathrm{CO}_{2}$ generation. We ascribed this effect to burst of the nanoparticles at the initial stage of their formation [63].

It should be noted that electrospray in solution is another technique that has a potential of producing calcium alginate particles with predefined size. This technique is based on collection of charged droplets of polymer solution in a glass with crosslinking solution (usually $\mathrm{CaCl}_{2}$ ). The control variables influencing particle size and size distribution are applied electric potential, collector distance, flow rate of polymer solution, its viscosity, conductivity, surface tension, concentration and polymer molecular weight. The possibility of variation of the size of the particles in wide limits has been demonstrated for alginate; however, the universal formula for size prediction still does not exist. Electrospray technique is reviewed in [147]; here we will discuss the possibilities of using aerosolization method for the synthesis of calcium alginate particles with predefined size. The discussion related to viscosity is valid for dripping method in general. Forced dissolution is also not limited to the particles produced using aerosolization method.

\section{Changing the viscosity of sodium alginate solution}

If alginate beads are produced by dripping sodium alginate into $\mathrm{CaCl}_{2}$ solution, their size depends on the drop size, which is influenced by diameter of a nozzle and viscosity of alginate solution. The size of air-dried beads was also shown to decrease with hardening time increase, possibly because of leak of the encapsulated drug (theophylline), and some increase with $\mathrm{CaCl}_{2}$ concentration was demonstrated. Higher temperature of $\mathrm{CaCl}_{2}$ solution in the interval of $5-20{ }^{\circ} \mathrm{C}$ improved air-dried bead sphericity. If the nozzle was placed too far above $\mathrm{CaCl}_{2}$ solution, wet beads flattened, and at high stirring speed they elongated. Fluidized-bed-dried as well as air-dried beads shrank during drying, but had much smoother surface and were less porous than freeze-dried ones. The two latter drying types did not cause the beads to become irregularly shaped. Theophylline, magnesium stearate and Kollidon K30 were admixed to sodium alginate solution before bead preparation. Bead size was in a millimeter range. The beads were intended for further incorporation into more complex drug delivery systems [148]. By means of dropwise addition of sodium alginate solution containing hemoglobin to $\mathrm{CaCl}_{2}$ solution with the aid of a syringe beads with an average diameter of about $2 \mathrm{~mm}$ were prepared, and at lower alginate concentration (and therefore lower viscosity) alginate microparticles with an average diameter of about $110 \mu \mathrm{m}$ were produced. $\mathrm{CaCl}_{2}$ concentration was simultaneously lowered (in order to avoid aggregation of the microparticles). In order to avoid hemoglobin oxidation observed at the time of encapsulation, its concentration was increased 10 times, and it led to particle size increase to $156 \mu \mathrm{m}$. Encapsulation and loading efficiencies for the latter case were $38 \%$ and $50 \%$, respectively. A tight interaction between alginate and hemoglobin affecting the release of oxygen was observed. The particles could possibly be applied for therapeutic approaches aimed at achieving the remediation of particular pathologic conditions [149]. For effervescent atomization of glycerol aqueous solutions it was shown that with increasing solution concentration (and therefore viscosity) the number of droplets of larger diameter increased and the droplet size histogram was wider [150]. In no case drops smaller than $0.25 \mathrm{~mm}$ were observed, although this might be due to the limitations of their measurement method employing a camera. In another study that also dealt with atomization of glycerol aqueous solutions but employed more advanced drop sizing technique it was similarly shown that the effect of viscosity acted to hinder the growth of instabilities in the liquid sheet, therefore resulting in longer break-up times and larger droplets [134]. A study of tapioca starch atomization also revealed that the reduction in viscosity not only eased the flow through the nozzle orifice but also boosted the surface wave oscillations and instabilities helping to break the solution stream into fine droplet spray patterns. Using chemically modified starch with opened ring the increase of variations in Sauter mean diameter across the spray centreline with an increase in solution viscosity and surface tension was demonstrated [135].

The viscosity of sodium alginate solution can be decreased in following ways:

- concentration decrease. The dependence of alginate viscosity on its concentration and shear rate is nonlinear [138].

- Addition of a supporting polymer that can form interpolymer complexes with sodium alginate, such as polyethylene oxide.

- $\quad$ Addition of a surfactant (Triton X-100 can also form a complex with alginate leading to double effect); it may also reduce the surface tension.

- $\quad$ Addition of a cosolvent being able to interact with sodium alginate, such as DMSO [151].

- Addition of electrolytes [45,139]. We have observed calcium alginate nanoparticle size decrease if sodium alginate used for their preparation was dissolved in physiological saline instead of water [63], but it may affect gel strength because the 
amount of calcium bound to alginate after addition of certain amount of $\mathrm{Ca}\left(\mathrm{NO}_{3}\right)_{2}$ solution to sodium alginate solution depends on calcium and sodium concentrations in solution. The volume fraction of hydrogel depends on the same parameters [152].

- Increase in temperature and/or in pressure, as shown for starch. The solution density and surface tension also decreased monotonically and reached their constant values above $90{ }^{\circ} \mathrm{C}$ [135]. We have observed calcium alginate nanoparticle size decrease if the nebulizer containing sodium alginate solution was heated [63].

- Using sodium alginate of lower molecular weight [151].

- $\quad$ Acid hydrolysis $[153,154]$. This is suitable only for alginic acid with subsequent neutralization to convert it to alginate.

- $\quad$ Alkaline degradation [45]. The viscosity of sodium alginate solution in water was shown to decrease during the first two weeks of storage because of hydrolysis, but this approach was not recommended [151]. Alginate hydrolysis may also occur at the time of its isolation from algae [152].

- $\quad$ Oxidation with $\mathrm{H}_{2} \mathrm{O}_{2}$ added $[154,155]$ or generated by interaction of reduced glutathione [155], cysteine [153,155], cysteamine, ascorbic acid [153,155], sodium ascorbate [155], phenylhydrazine, dihydroxymaleic acid, thioglycolic acid, sulfite, dithionite, phloroglucinol, sodium hydrogen sulfide, 'fucosan' (endogenous phenolic compounds of brown algae) [153], etc. with atmospheric oxygen. Further reaction of hydrogen peroxide with the reducing compounds generates free radicals oxidizing alginate more rapidly. Ferrous ions can also be used for the same purpose because they decompose hydrogen peroxide leading to the same radicals, but at high concentrations they form gel with alginate [153]. When polymannuronate blocks having $\sim 40$ sugar units produced by sodium alginate treatment with $0.5 \mathrm{M} \mathrm{HCl}$ at $100^{\circ} \mathrm{C}$ for 8 hours were further treated with $5 \% \mathrm{H}_{2} \mathrm{O}_{2}$ for 30 min, oligomers having 1-15 sugar residues were formed [154]. But small fragments of alginate are not suitable for gelation.

- Chemical modification of alginate can both increase and decrease its viscosity, but it is usually accompanied by chain destruction and at high substitution degrees makes gelation impossible [45].

\section{Drop evaporation in preformed aerosol}

The total output from a nebulizer is made of aerosol and a significant component of water vapor. As the evaporative component increases, the cumulative solute output drops. The concentration of solute in the nebulizer solution increases with operating time in a nonlinear manner. At the same time the temperature of nebulizer solution can drop up to $12{ }^{\circ} \mathrm{C}$ lower during operation, but then equilibrium is reached. As the driving air pressure is increased, the equilibrium temperature is lowered, but the time to reach equilibrium remains constant. Increase in the ambient humidity of the supply air and decrease in the volume flow rate reduce solvent evaporation [156]. Heating of preformed diluted aerosol in order to induce water evaporation and therefore decrease drop size was proposed as a method to convert aerosols with micrometer-sized drops produced by common medical nebulizers to those with nanosized drops. The best heater type was air jacketed tube, and preheated air was flowing through the outer line in the opposite direction of the aerosol stream. In the case of aerosol of $0.1 \%$ albuterol sulfate it was possible to convert aerosol having mass mean aerodynamic diameter of $4.69 \mu \mathrm{m}$ to this having mass mean geometric diameter of $0.43 \mu \mathrm{m}$. Dry nanoparticles of predetermined sizes can be formed by reducing drug concentration. The possibility to predict final drop size by means of computational fluid dynamics was demonstrated [157].

\section{The attempts to predict aerosol drop size distribution}

For several atomizer types there are equations allowing to predict Sauter mean diameter of the aerosol droplet. This, in turn, allows one to predict drop size distribution. The most important input parameter is viscosity because it has a cohesive effect and impedes the disintegration mechanisms leading to droplet formation. Other parameters for pressure-jet atomizers are liquid surface tension, air density, liquid density, discharge orifice diameter, injection pressure differential across the nozzle, half spray angle, and liquid mass flux. For pre-filming airblast atomizers the first three parameters are needed along with air velocity and air/liquid ratio; for plain-jet airblast atomizers discharge orifice diameter is needed as well [158]. Another model allows predicting number-based dimensionless drop size distribution near the region of their formation, i.e. not that of the drops actually reaching $\mathrm{CaCl}_{2}$ solution. The input parameters are gas velocity, liquid velocity at the atomizer exit, gas density, liquid density, surface tension, drop mass mean diameter, linear growth rate of the sinuous or varicose waves, origin shift parameter, dimensionless critical diameter, and at least two more parameters [159]. A more advanced model based on maximum entropy principle, as were the ones mentioned before, is aimed at prediction of drop size distribution at the end of the primary breakup region, i.e. still not of that of final drops. At least the following input parameters are needed: mass flow rate, mean velocity of jet in nozzle outlet, gas density, liquid density, surface tension, drop mass mean diameter, shape factor for velocity profile, Reynolds number, breakup length and thickness of the liquid sheet [136]. Although some of these parameters can be substituted for other ones, unfortunately in real experiment of alginate aerosolization they are still very difficult to measure, and it makes the prediction impossible. In general, the predictive power of maximum enthropy method is rather poor; it produces good agreement with the experimental data only after adjustment of parameters or if the representative drop diameter is known from direct measurements [160]. Obviously, in these two cases the method relies completely on the data it must predict, i.e. it becomes just a way of fitting the existing data and is no longer predictive. Moreover, the equations do not take into account the possibility of drop size change resulting from water evaporation discussed above. There is also a discrete probability function approach aimed at predicting drop size distribution from the fluctuating initial conditions, but it is again limited to primary atomization and requires prior knowledge of probability density function of the fluctuating initial conditions. These functions cannot be currently 
determined [160], i.e. the method cannot be used in practice. One more model based on the consideration of instabilities during the atomization of a liquid jet by a co-flowing gas stream allows to predict the ratio of Sauter mean diameter of the final drops to the liquid orifice diameter if the gas stream is laminar. The input parameters are gas density, liquid density, ratio of gas boundary level thickness at the gas discharge plane to the liquid orifice diameter, Weber number, Ohnesorge number, Reynolds number and mass flux ratio. The latter 4 parameters, in turn, can be calculated from some additional parameters. However, two coefficients in the final equation must be determined experimentally [134]. It should also be noted that neither of the models discussed above has been verified for the case of nanosized drops.

Despite the problems with exact prediction of drop size distribution, a qualitative trend can be revealed. The study of effervescent atomization of aqueous solution of glycerol demonstrated nonlinear decrease of Sauter mean diameter of aerosol drops with the increase of air to liquid mass flow rate ratio [150], although the dependence seems to reach a plateau at ratios higher than $\sim 0.5$.

\section{Forced dissolution of calcium alginate particles}

As can be seen from the discussion above, the synthesis of calcium alginate particles with exactly predefined size by means of aerosolization method is very difficult. However, it is possible to produce particles having a size larger than needed and then use their controllable dissolution for fine tuning the size. All these possibilities, except the use of EDTA (see below), are still unexplored.

Calcium alginate hydrogel can be dissolved by treatment with EDTA, as reported for low weight alginate produced by sodium alginate irradiation with cobalt source [75]. We have shown the possibility of using this reaction for adjustment of the size of preformed calcium alginate nanoparticles produced from commercially available low viscosity sodium alginate [63]. Sodium citrate can also be used for the same purpose, and the relation curve between the gelatin/alginate/collagen hydrogel degradation time and mole ratio citrate: alginate was obtained. If the amount of sodium citrate was greater than that of alginate, the degradation was too quickly to be controllable. Biocompatibility of sodium citrate was proven for human corneal epithelial cells encapsulated in this hydrogel. Treatment with sodium citrate may help to improve the alginate bioink system for the application of 3D bioprinting in tissue engineering. The printed cells could not degrade the surrounding alginate matrix themselves, causing them to remain in a poorly proliferating and non-differentiating state [161]. Phosphate ion was also demonstrated to dissolve calcium alginate $[138,145]$ thus accelerating the release of encapsulated rhodamine 6G [146]. Phosphate buffered saline accelerated protein release from alginate particles by their degradation $[112,126,138]$. The dissolution of chitosan-covered dried calcium alginate beads with a size of $\sim 1 \mathrm{~mm}$ in simulated intestinal fluid $\left(\mathrm{NaOH}+\mathrm{KH}_{2} \mathrm{PO}_{4}\right)$ was complete after 1 hour of incubation [118]. Complete dissolution of alginate beads with an average diameter of about $2 \mathrm{~mm}$ during 30 minutes of incubation in phosphate buffered saline at $\mathrm{pH} 7$ under stirring in the presence of alginate lyase was reported. Before the dissolution study, the beads had been washed with phosphate buffered saline 3 times in order to remove excess $\mathrm{Ca}^{2+}$ [149]. It should be pointed out that some authors claim their alginate particles as demonstrating pH-dependent drug release while using phosphate buffers for alkaline $\mathrm{pH}$, but in fact the presence of phosphate is the main cause of drug release because of particle dissolution. Phosphate buffers are often used as 'simulated intestinal fluid', sometimes even without providing their composition.

Non-crosslinking cations compete with calcium ions for carboxyl groups of alginate and therefore can disrupt electrostatic interactions between alginate chains. The affinity of the cations to alginate grows in the following order: $\mathrm{K}^{+}<\mathrm{Na}^{+}<\mathrm{Mg}^{2+}$. But this does not lead to bead disintegration, except at high salt concentration (50mM for $\mathrm{NaCl}$ or $\mathrm{KCl}$ and $10 \mathrm{mM}$ for $\mathrm{MgCl}_{2}$ ). At lower salt concentrations bead swelling occurs if the beads were previously dried. This happens because at low concentrations the salts disrupt only dimer-dimer associations, but individual egg-box dimers remain intact. These dimer-dimer associations appear during bead drying and do not exist in fresh beads. Bead swelling in pure water did not occur. The beads used were prepared by dripping sodium alginate solution into $\mathrm{CaCl}_{2}$ solution under stirring with subsequent incubation in $\mathrm{CaCl}_{2}$ solution. Bead diameter was $\sim 3.2 \mathrm{~mm}$, the bead weight was $17.9 \mathrm{mg}$ before drying and $0.6 \mathrm{mg}$ after drying [162]. The applicability of this method to nanoparticles was also shown $[73,95]$. Alginate beads were chosen as a model system in the study of the changes in the food microstructure during drying in order to improve the quality of dried foods [162].

To our opinion, aerosolization method is preferable if calcium alginate nanoparticles with adjustable size and narrow size distribution, without the admixture of microparticles and without surfactants are needed in moderate quantities. (For beads and microparticles it may be easier to use dripping method). Although theoretical prediction of nanoparticle size has not been achieved yet, it is possible to obtain calibration data for a particular nebulizer. Moreover, particle size can be decreased in a controllable way by forced dissolution, as discussed above. This method allows fine adjustment of particle size after proper calibration. Another possible method of producing nanoparticles with partially predictable size is electrospray, but it is less safe because of high voltage.

\section{Encapsulation of cyclodextrin inclusion complexes in alginate particles}

Cyclodextrins are torus-like macro-rings built up from glycopyranose units (Figure 7). They have hydrophilic sites on the outer surface and a hydrophobic cavity inside. The diameter of the one end of the cavity is larger than that of the opposite end. There are numerous cyclodextrins, the most important ones being $\alpha-, \beta$ and $\gamma$-cyclodextrins, comprising 6,7 , and 8 glucose units and having cavity diameter of 4.7-5.3, 6.0-6.5 and 7.5-8.3 $\AA$, respectively. In an aqueous solution, the water molecules inside the cavity can be readily substituted by appropriate 'guest molecules' which are less polar than water. Upon dissolving the inclusion complexes formed, an equilibrium is established between dissociated and associated species [163]. 


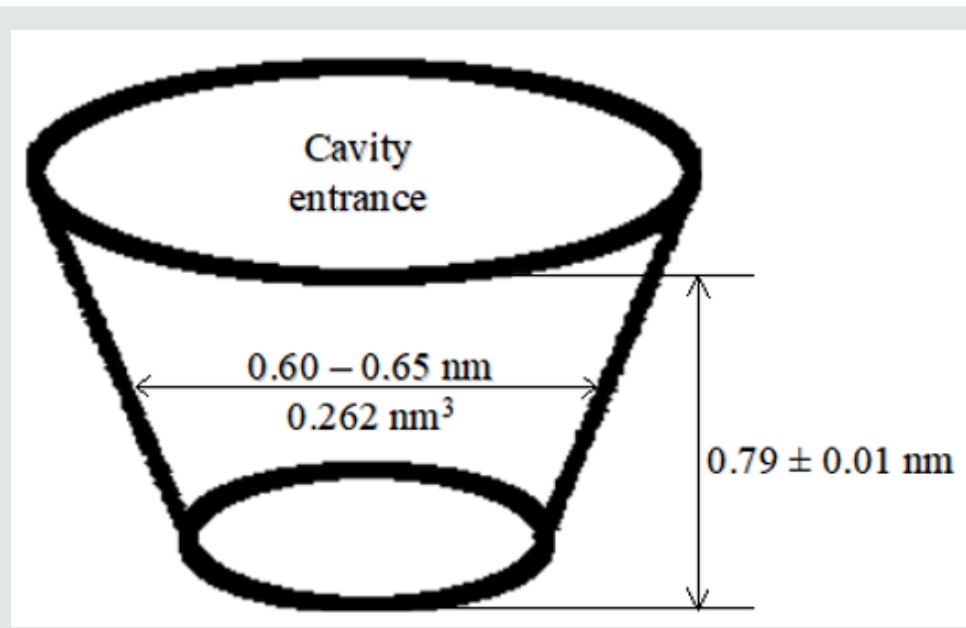

Figure 7: $\beta$-cyclodextrin molecule.

Cyclodextrins can even form inclusion complexes with various volatile substances, although most of these complexes are rather unstable in the presence of water. The results of spray-drying complex mixtures of numerous flavors briefly incubated with cyclodextrins showed that $\gamma$-cyclodextrin was generally the most effective in retaining the flavor compounds during spray-drying, but the losses of volatilities on storage were greatest for $\gamma$-cyclodextrin and least in the case of $\alpha$-cyclodextrin [164]. The synthesis of an inclusion complex of eugenol and $\beta$-cyclodextrin and its antifungal activity helping to preserve fruits has been reported [165]. Allyl isothiocyanate was shown to be released continuously frominclusion complexes with $\alpha$ - and $\beta$-cyclodextrins for 120 hours even at $98 \%$ relative humidity, but initial burst release was observed [166]. Both complexes had fungicidal activity against Penicillium expansum, and the complex with $\beta$-cyclodextrin displayed bactericidal activity against E.coli and Listeria monocytogenes as well [167]. Inclusion complexes of 2-nonanone and $\beta$-cyclodextrin were active against fungal pathogen Botrytis cinerea, which led to the grey rot on strawberries, blueberries, raspberries and table grapes [168]; an inclusion complex $\beta$-cyclodextrin-hexanal was active against Colletotrichum acutatum, a fungus that is among the most common cases of berry decay [169]. Three techniques of the synthesis of hydroxypropyl- $\beta$-cyclodextrin-capsaicin inclusion complex were reported [170-172], although the aqueous solutions of the complex still contained free capsaicin [172]. Even acetaldehyde, a rather small molecule, was successfully encapsulated in $\beta$-cyclodextrin, and the inclusion complexes were active against postharvest decay fungus Alternaria alternata [173]. A more advanced technique proposed was acetaldehyde-diethylacetal encapsulation by hydroxypropyl$\beta$-cyclodextrin. The inclusion complex was stable under normal shelf-storage conditions for one year, but immediately decomposed on contact with water [174]. However, smaller molecules show weaker binding. Similarly modified $\alpha$ - and $\beta$-cyclodextrins were shown to entrap aniline vapor in higher amounts than benzene [175]. Benzene binds much weaker than tert-butylbenzene with both native and carboxymethyl- $\beta$-cyclodextrin, as shown by molecular dynamics simulations [176]. The extracts of cinnamon bark and clave bud without isolation of the essential substances were used for encapsulation, and the formation of microparticles of the inclusion complexes by means of freeze-drying was reported. The antibacterial activity of the complexes against Salmonella enterica serovar Typhimurium LT2 and Listeria innocua was higher than that of the nonencapsulated extracts [177]. Numerous other examples of complexes of cyclodextrins with volatiles, the methods of preparing them, the methods by which the complex formation can be evidenced, the applications of the complexes and some other aspects are reviewed in [178].

It can be expected that alginate encapsulation of the inclusion complexes instead of free molecules may give an opportunity for additional retardation of their release.

\section{The published examples of the immobilization of cyclodextrin inclusion complexes in alginate}

The cationic polymer produced from $\beta$-cyclodextrin, epichlorohydrin and choline chloride was shown to form complexes with insulin, despite insulin molecules being too bulky to be wholly included into the cavity of cyclodextrin. Dropwise addition of sodium alginate solution containing the complex to $\mathrm{CaCl}_{2}$ solution followed by chitosan addition led to beads [179]; the addition of $\mathrm{CaCl}_{2}$ and then chitosan [180] or trimetylchitosan [181] to sodium alginate solution containing the complex led to nanoparticles $[180,181]$. The beads had irregular shape and relatively smooth surface with some wrinkles due to lyophilization. The encapsulation efficiency was up to $72 \%$, being influenced by cationic polymer composition and amount, complexation time and $\mathrm{CaCl}_{2}$ concentration. The beads released around $20 \%$ of insulin in simulated gastrointestinal fluid [179]. Their size was in the millimeter range, as stated later [180]. They were initially claimed as a promising system for improving insulin oral delivery efficiency [179], but they did not show significant improvement of insulin release efficiency in the following trials [180]. Most nanoparticles coated with chitosan had irregular shape and size in the range of $100-200 \mathrm{~nm}$. Encapsulation and loading efficiencies were up to $87 \%$ and $9.5 \%$, respectively [180]. After freeze-drying in the presence of sucrose the nanoparticles coated with trimethylchitosan were spherical or subspherical. Aggregation was not observed. Their size was influenced by $\beta$-cyclodextrin, sodium alginate and $\mathrm{CaCl}_{2}$ concentrations, and could 
be varied from $187 \pm 20 \mathrm{~nm}$ to $2393 \pm 1219 \mathrm{~nm}$. The same parameters influenced polydispersity index. For optimized formulation it was 0.362 . Encapsulation efficiency was more than 93\%. Higher encapsulation efficiency was associated with higher concentrations of trimethylchitosan and $\beta$-cyclodextrin as well as with lower $\mathrm{pH}$ of $\beta$-cyclodextrin. Loading efficiency was $5 \%$ [181]. The nanoparticles coated with chitosan released $48 \%$ of encapsulated insulin during 2 hours of incubation in simulated gastrointestinal fluid, and $40 \%$ of insulin was released during subsequent incubation in simulated intestinal fluid (containing phosphate) for 4 hours. In the case of uncomplexed insulin encapsulation these values were $60 \%$ and $18 \%$, respectively, and the mean particle size exceeded $700 \mathrm{~nm}$. The release profile could be controlled by varying the molecular structure of the cationic polymer besides the alginate/chitosan mass ratio. The latter parameter along with cationic polymer content also affected the mean particle size [180]. The nanoparticles coated with trimethylchitosan released $5 \%$ and $94 \%$ of insulin in these media, respectively. Burst release of most of encapsulated insulin occurred in simulated intestinal fluid. Insulin permeation across Caco-2 cell monolayer from apical to basolateral side was significantly increased by these nanoparticles if compared with the nanoparticles prepared with unmodified $\beta$-cyclodextrin and with free insulin. $1 \mathrm{ml}$ of medium containing encapsulated insulin at $0.4 \mathrm{mg} / \mathrm{ml}$ was used for the permeability studies, and almost $9 \%$ of insulin was transported for $240 \mathrm{~min}$. The monolayer was grown on polyethylene terephthalate membrane filters with a pore size of $0.4 \mu \mathrm{m}$ [181]. The system provided enhanced insulin stabilization in the presence of pepsin [180]. The nanoparticles can be considered as a good candidate for oral insulin delivery $[180,181]$.

Covalent binding of $\beta$-cyclodextrin to alginate via linkers was also reported. In the case of ethylenediamine linker the binding was archived by means of carbodiimide chemistry. In contrast, binding through 1,6-hexanediamine linker was carried out in a more complex way, beginning with turning sodium alginate into alginic acid with its subsequent neutralization with tetrabutylammonium hydroxide. Cyclodextrin was activated with tosyl group prior to the synthesis. Addition of Pluronic F108 to the modified alginate at different ratios yielded hydrogels by complexation of cyclodextrin with its polypropylene glycol residues. The goal of the study was to create a host biomacromolecule, viz. alginate with grafted $\beta$-cyclodextrin, and incorporate a difunctional guest molecule in order to create physically crosslinked, moderately stiff hydrogels. The hydrogels could release encapsulated bovine serum albumin for up to 14 days, and about $40 \%$ of encapsulated protein were released in phosphate buffered saline. Encapsulation was carried out by protein addition to preformed hydrogels, because they could swell and incorporate the protein. The release depended on linker type and ratio of Pluronic F108 to alginate. Incubation of the hydrogels at $4 \%$ and $6 \%(\mathrm{w} / \mathrm{v}$ ) for 24 hours with primary human

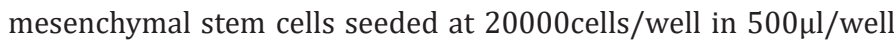
of the growth medium showed no cytotoxicity. Biologically relevant mechanical properties of the hydrogels would be advantageous for biomedical applications such as drug delivery and cell transplantation [182].
It should be noted, however, that in the case of invertase immobilized in calcium alginate beads addition of $\beta$-cyclodextrin led to bigger pores and higher rate of release. The encapsulation efficiency dropped from $92 \%$ to $82 \%$. The beads were prepared by dripping sodium alginate solution containing the enzyme into $\mathrm{CaCl}_{2}$ solution in acetate buffer ( $\mathrm{pH}$ 3.8) containing $\beta$-cyclodextrin and trehalose. Trehalose protected the encapsulated enzyme during freezing, drying and thermal treatment, and $\beta$-cyclodextrin protected it during bead formation. The size of the beads depended on the drying technique and the composition, but was in the millimeter range. The enzyme release depended on $\mathrm{pH}$ and drying technique, but it took several hours in all the cases. The study was aimed at providing new alternatives for enzyme stabilization and release management in order to reduce costs and increase the productivity of the technological process employing invertase [183]. For spherical microparticles produced by spray drying the solutions of raffinose or trehalose containing hydroxypropyl- $\beta$ cyclodextrin in methanol: n-butyl acetate mixture the increase of porosity with cyclodextrin weight fraction up to $40 \%$ with subsequent decrease was revealed. The addition of cyclodextrin resulted in 2-times decrease of mass median aerodynamic diameters to about $2 \mu \mathrm{m}$. This effect was ascribed to high porosity diminishing the contact areas between particles and therefore hindering aggregation. The preparations of microparticles had improved physical characteristics, physicochemical stability and aerosolization, suggesting their potential as carrier system for pulmonary delivery of biomolecules [184].

\section{The unrealized possibilities of further improvement of small molecule encapsulation and release retardation}

In theory, there is a way of encapsulation of small molecules in cyclodextrins by means of cavity size tuning, but there has been no attempt to combine these techniques with the encapsulation of the resulting inclusion complexes in alginate particles. Enzymatic synthesis of cyclothiomaltins, viz. cyclo- $\alpha(1 \rightarrow 4)$-4-thiomaltotetraoside, -thiomaltopentaoside and -thiomaltohexaoside has been reported [185]. They must have smaller cavities than that of $\alpha$-cyclodextrin. Peracetates of cyclic $(1 \rightarrow 6)$ - $\beta$-D-glucan trimer and tetramer were chemically synthesized from derivatized linear tri- and tetrasaccharides, and their cavity hole size was calculated as 1 and $3.3 \AA$, respectively [186]. It should be noted, however, that the cavity may have dynamic nature. For example, a glycophane containing two benzoic acid spacers displayed great conformational flexibility [187].

Several other possibilities of cyclodextrin cavity tuning without decreasing its size exist. A single oxygen atom in the ring skeleton of $\beta$-cyclodextrin can be replaced by a disulfide bond in two different configurations making the cavity asymmetric [188]. In permethylated $\alpha$ - and $\beta$-cyclodextrins $\beta$ - $(1,4)$-glucosidic bond can be introduced instead of $\alpha$-(1,4)-glucosidic one to alter the cavity shape $[189,190]$. This technique was extended to 2,6-di0 -methylated $\alpha$ - and $\beta$-cyclodextrins [190]. The $\alpha$-cyclodextrin analogues having a thiophene or triazole fragment inserted in the ring as well as $\alpha$-, $\beta$ - and $\gamma$-cyclodextrin analogues carrying fragments with triple bonds were reported [191]. 
A theoretically possible way to retard the release of encapsulated molecules from the inclusion complexes is steric hindrance introduction. An interesting example is the synthesis of hyperbranched polyglycerol grafted from $\beta$-cyclodextrin. This molecule can be further modified with amine groups [192]. But this technique was not intended for the combination with alginate.

In conclusion, the encapsulation of drugs in the form of inclusion complexes with cyclodextrins appears rather promising and underexplored. This is especially important for unstable or small molecules provided they can form the inclusion complexes. Cavity size tuning and steric hindrance introduction will probably be of limited applicability because they make the synthesis expensive, and biocompatibility of the resulting complexes is questionable. Another problem is that the instability of many inclusion complexes in water may lead to their decomposition at the time of alginate particle formation. Nevertheless, the use of cyclodextrins will surely provide additional power to the encapsulation methods.

\section{The Decrease of Alginate Pore Size}

Calcium alginate pore size is usually assumed to be in the range of 5-200nm. It is a so common assumption that reviews [193] may give it citing other reviews, and sometimes [42] it is given even without reference. Nevertheless, it is not always the case. The pores of calcium alginate hydrogel produced by treatment of spin-coated sodium alginate with $\mathrm{CaCl}_{2}$ were found to have typical diameters of $50-300 \mathrm{~nm}$. The study dealt with calcium alginate hydrogel as a model of extracellular polymeric substances because of similar physicochemical properties and architecture [194]. In another study, alginate was spin-coated onto a poly-L-lysine-coated glass slide and then treated with $\mathrm{CaCl}_{2}$. Pore size range of $4-18 \mathrm{~nm}$ was observed. Moreover, their review of previously published literature indicated that pore sizes from $4 \mathrm{~nm}$ up to $10.9 \mu \mathrm{m}$ had been reported [195]. In any case, there are some pores having much larger size than that of protein molecules. This is the main cause of burst release of encapsulated substances. Obviously, the situation with low molecular weight substances is even much worse than with proteins. The diffusion coefficients of glucose and ethanol in $2 \%$ calcium alginate are only slightly lower than in water. Their diffusion is mainly controlled by the rate at which they diffuse through water occupying the pores. Glucose and ethanol concentrations have no effect on the respective diffusion coefficients. Their decrease with increasing alginate concentration is probably due to a decrease in the number and length of the pores rather than a decrease in the pore diameters. The diffusion coefficients were needed to model reaction and diffusion in immobilized cell reactors [196].

The size of the pore openings can be effectively reduced by chitosan coating, as revealed by the study dealing with hydrogel film acting as a model of an immuno-isolation membrane [195]. Besides this effect, chitosan layer provides an additional diffusional barrier. Another option is the use of additional crosslinking agents.

In one example, alginate beads containing immobilized xylanase were produced by extruding sodium alginate solution containing the enzyme through a needle to $\mathrm{CaCl}_{2}$ solution, and then additional overnight incubation in $\mathrm{CaCl}_{2}$ solution was carried out. The beads were then incubated with $1 \%$ glutaraldehyde at $\mathrm{pH}$ 6.0 for 90 minutes under stirring and washed with acetate buffer. The immobilization shifted the enzyme $\mathrm{pH}$ optimum from $\mathrm{pH} 5.0$ to 5.5, and treatment with glutaraldehyde shifted it further to $\mathrm{pH}$ 6.0. Encapsulation efficiency before and after glutaraldehyde application was $41 \%$ and $91 \%$, respectively. Treatment with glutaraldehyde almost did not provide any additional enzyme stabilization at acidic or alkaline pH. Enzyme thermostability, stability during storage and in the presence of chemicals $\left(\mathrm{HgCl}_{2}\right.$, etc.) was increased, but the enzyme was operating more slowly. No additional shift of temperature optimum was recorded, but there was a shift to higher temperatures if compared with the free enzyme. After 4 reaction cycles the enzyme activity started to decrease, and after 12 cycles most of the enzyme leaked out. It was also washed out by bead treatment with sodium dodecyl sulfate [42]. Therefore, there was no covalent linkage of the enzyme with alginate. The effect of glutaraldehyde was explained by enzyme crosslinking. Alginate crosslinking was not considered, although it was likely to happen. The beads could be efficiently used for fruit juice clarification and yield improvement [42].

In another example, sodium alginate solution containing diclofenac sodium [197] or neem seed oil [198] was added dropwise into methanol containing $1 \%$ glutaraldehyde and $1 \%$ of $10 \mathrm{M} \mathrm{HCl}$ [197] or $1 \%$ of $1 \mathrm{M} \mathrm{HCl}$ [198]. This resulted in formation of almost spherical beads with nonporous smooth surface and size 1.2-1.6mm [197,198]. Crosslinking time did not influence the particle size but decreased the release rate and bead swelling in water. Diclofenac release was studied at $\mathrm{pH} 1$ for 3 hours and then at pH 7.4 [197], and that of neem seed oil in $0.1 \%$ Tween 80 [198]. The beads formed at $40{ }^{\circ} \mathrm{C}$ showed lower release rate than those formed at $25^{\circ} \mathrm{C}$, but this effect was less prominent [198]. Both parameters caused encapsulation efficiency decrease because of drug leaching during crosslinking. Increasing concentration of the substance being encapsulated in sodium alginate solution decreased encapsulation efficiency and increased release rate [197,198]. The longest diclofenac release time achieved for the beads with encapsulation efficiency of $>55 \%$ was $300 \mathrm{~min}$. Controlled release system is needed for diclofenac because of its short biological half-life and side effects such as ulcer bleeding or perforation of intestinal wall [197]. For the beads loaded with neem seed oil these parameters were $>72 \%$ and 25 hours, respectively. There was no chemical interaction between neem seed oil and glutaraldehyde. The aim of the study was to create a controlled release formulation of a pesticide with a minimum residual toxicity [198].

Other techniques of crosslinking alginate with glutaraldehyde exist, but they were reported for alginate fibers or membranes, not for particles:

- $2 \mathrm{ml}$ of glutaraldehyde and $2 \mathrm{ml}$ of concentrated $\mathrm{HCl}$ in $180 \mathrm{ml}$ of $1: 2(\mathrm{v} / \mathrm{v})$ water-acetone mixture for $\sim 10$ hours, then wash with water and drying [199]

- $\quad 2.5 \mathrm{ml}$ of glutaraldehyde and $2.5 \mathrm{ml}$ of concentrated $\mathrm{HCl}$ in 30:70 water:acetone mixture for $\sim 12-14$ hours, than wash with water and drying at $40{ }^{\circ} \mathrm{C}[200,201]$ 
- $\quad 5 \%(\mathrm{v} / \mathrm{v})$ glutaraldehyde and $1 \%(\mathrm{v} / \mathrm{v}) \mathrm{HCl}$ in 90:10 methanol: water for 4 hours, than wash with water [202]

- $\quad 250 \mathrm{mM}$ glutaraldehyde and $0.05 \mathrm{M} \mathrm{HCl}$ in $50 \%$ ethanol for 48 hours at room temperature, than wash with water and drying under reduced pressure [151]

- $\quad 5-20 \%(\mathrm{v} / \mathrm{v})$ glutaraldehyde (optimized concentration was $10-15 \%)$ and $0.05 \%(\mathrm{v} / \mathrm{v}) \mathrm{HCl}$ in acetone at $40{ }^{\circ} \mathrm{C}$ for 48 hours, than wash with methanol, immersion in methanol for 24 hours at $40{ }^{\circ} \mathrm{C}$, and vacuum drying. Some aldehyde residues were pendent from alginate chains instead of actual crosslinking [203]

- $\quad \leq 3 \%$ glutaraldehyde and $0.1 \%$ of $35 \% \mathrm{HCl}$ in dioxane at 50 ${ }^{\circ} \mathrm{C}$ for 4 hours with subsequent neutralization in 80:20 (v/v) methanol/0.4M NaOH mixture at room temperature for $20 \mathrm{~min}$, then wash with $80 \%$ methanol. In dioxane better crosslinking efficiency than in methanol or acetone was achieved, but $<10 \%$ of alginate still remained water soluble [204].

Dropwise extrusion of sodium alginate solution into $0.05 \mathrm{M} \mathrm{HCl}$ with subsequent incubation for 4 hours resulted in beads having mean diameter of $2.9 \mathrm{~mm}$. The beads were crosslinked in a solution containing $1.58 \mathrm{M}$ glutaraldehyde (3.16 $\mathrm{M}$ inside the beads) and $0.38 \mathrm{M} \mathrm{HCl}[205,206]$. Control of reaction kinetics allowed to adjust the mean pore size from $720 \mathrm{~nm}$ to less than $35 \mathrm{~nm}$. However, microscopy studies revealed pore size distribution of $25-200 \mathrm{~nm}$ in the latter case [205]. In one example, pore size changed from less than $0.1 \mu \mathrm{m}$ in acid solution to almost $0.5 \mu \mathrm{m}$ in alkaline solution because of reversible gel swelling [206]. The time required to crosslink available hydroxyls could range from 1 to over 10 hours depending on the reaction conditions. The reactivity of guluronic and mannuronic acid residues was shown to be equal. The reaction had zero, second and first order with respect to alginate concentration, glutaraldehyde concentration, and acid concentration, respectively [205]. The addition of aprotic solvent (acetone, dioxane, dimethyl sulfoxide) to the crosslinking medium enhanced the reaction rate, while the use of protic solvents (alcohols) decreased it [206]. The reaction rate also increased with alginate molecular mass and with temperature. At room temperature the expected maximum crosslinking density ( $\sim 5$ monomeric units between crosslink points) was not reached because of glutaraldehyde loss in a parallel oxidative degradation reaction, but at $40-50{ }^{\circ} \mathrm{C}$ it was reached. At initial glutaraldehyde concentration less than $0.9 \mathrm{M}$ the reaction was more diffusional controlled [205]. Glutaraldehyde can also turn into polycyclic or $\alpha, \beta$-unsaturated polyglutaraldehyde. These products may react further with alginate to form complex network structures. Some fraction of glutaraldehyde reacted only with one hexauronic acid residue, i.e. it was not involved in crosslinking [206]. Detailed mechanism of the crosslinking reaction was also elucidated [205]. Crosslinked alginate can potentially be used as a pulsatile oral delivery vehicle for protein therapeutics, as a superabsorbent for environmental cleanup application, or as a scaffold for tissue engineered implants [205,206].

A technique of crosslinking calcium alginate nanofibers using epichlorohydrin was reported. In this case, dried nanofibers were soaked in 50\% ethanol containing 5M epichlorohydrin and $0.1 \mathrm{M}$ $\mathrm{NaOH}$ for 24 hours. The reaction was stopped by the addition of $5 \mathrm{M} \mathrm{HCl}$. The nanofibers were washed with $75 \%$ ethanol, then with water and dried under reduced pressure. The approach may be beneficial to the production of nanofibrous matrices mimicking the native extracellular matrix for a wide range of tissue engineering applications [151].

Tannic acid can be used as an additional noncovalent crosslinker. It was demonstrated for the nanoparticles produced by dropwise addition of $\mathrm{CaCl}_{2}$ to the solution containing sodium alginate, tannic acid and diltiazem hydrochloride under stirring. The resultant suspension was aged for $10 \mathrm{~min}$ followed by sonication and centrifugation, and the nanoparticles were resuspended in water and sonicated again. Drug encapsulation efficiency was found to increase with tannic acid concentration from $15 \%$ to $66 \%$. Blank calcium alginate nanoparticles had the diameter of $475 \mathrm{~nm}$ with polydispersity index of 0.56 and zeta potential of $-23 \mathrm{mV}$. Introduction of drug and tannic acid caused only a slight change of these parameters. All the nanoparticles were discrete and spherical. Tannic acid reduced the drug burst release within $30 \mathrm{~min}$ in $0.1 \mathrm{M} \mathrm{HCl}$ from $44 \%$ to $11 \%$. After 2 hours in this acidic medium the nanoparticles were transferred to phosphate buffered saline, and tannic acid reduced the cumulative drug release from $100 \%$ to $5 \%$ and prevented disintegration of the nanoparticles. Diltiazem hydrochloride is used in the treatment of hypertension, asthma and arrythmia, but in the case of non encapsulated drug frequent administration is required [207]. We tried to produce crosslinked calcium alginate nanoparticles using aerosolization of sodium alginate solution containing glutathione (a drug being encapsulated) and phytic acid into $\mathrm{CaCl}_{2}$ solution, but this technique did not prevent burst release if sodium alginate solution $\mathrm{pH}$ was not readjusted. Nevertheless, the attempt of using sodium cromoglicate instead of phytic acid led to more prolonged glutathione release even without $\mathrm{pH}$ adjustment, but overnight incubation of the nanoparticles in $1.11 \%$ glutaraldehyde in $0.4 \% \mathrm{HCl}$ gave better results [63].

Several other methods of alginate crosslinking exist. One of them is based on activation of carboxyl groups of tetrabutylammonium alginate with subsequent crosslinking them using a diamine in the presence of triethylamine taken as catalyst. Another one is based on linking protonated carboxyl groups to hydroxyl groups using the carbodiimide coupling reagent. There is also a method in which partial oxidation of hydroxyl groups to aldehyde ones with subsequent crosslinking with adipic dihydrazide is used [45]. Methacrylated alginate can form hydrogels by free radical polymerization. If alginate with immobilized ligands is used for cell encapsulation, the cells can act as crosslinkers themselves, but the network exhibits low strength and toughness, as reviewed in [208].

Therefore, coating and additional crosslinking do lead to pore diameter reduction. However, no universal solution of the burst release problem exists. Crosslinking effectively reduces mean pore size, but some portion of big pores remains or appears during particle swelling. Moreover, drugs of low molecular weight may leak out during crosslinking. Coating is a powerful method. Besides 
porosity decrease, it allows surface modification and charge adjustment. However, it ultimately increases particle size.

\section{Conclusion}

The characteristics of alginate such as its natural origin, abundance in nature, biocompatibility, biodegradability and hydrogel formation upon crosslinking with calcium ions make it a perfect base for micro- and nanoparticles for drug delivery and other applications. This conclusion is supported by numerous publications dealing with them, indicating significant interest of the scientific community to this versatile biopolymer. Moreover alginate can be combined with additional biocompatible drug release retardants, e.g. cyclodextrins, creating additional largely unexplored possibilities for its clinical, agricultural and other use.

However, a question arises: why alginate nanoparticles are still not in use in real clinics, despite their great potential? Bulk alginate is already in use as an excipient in tablets, component of wound healing tapes and even as a dietary supplement, but its nanoparticulate form is not.

A very important or even the main reason may be the burst release of the encapsulated drugs. This is the main problem in creating alginate-based drug formulations, and it is caused by inherent properties of calcium alginate hydrogel - porosity and reversibility of gelation. Both problems may be overcome by covalent crosslinking and coating. However, chemically modified alginate is no longer natural and biodegradable, and the currently available coating methods either do not provide sufficient drug release retardation or lead to a so huge increase of the particle size that it becomes dangerous because of the possible embolism. Moreover, there is no method of producing small (i.e. not submicronsized) calcium alginate nanoparticles with predictable size and guaranteed narrow size distribution without employing chemical surfactants, the safety of which is questionable. One should also remember that initially 'small' dry nanoparticle can become 4 times larger after swelling. Another problem is that hydrogel strength increases with alginate polymerization degree, but alginate of high molecular weight is far above the renal clearance threshold.

Nevertheless, the above-mentioned concerns do not override the wide perspectives of clinical use of alginate particles. New investigations must be directed to the use of alginate particles in combination with natural drug release retardants, improvement of coating, switching to natural surfactants (e.g. lecithin), the use of more degradable alginate (e.g. partially oxidized), development of new methods of synthesis of nanoparticles with predefined size distribution, and to more extensive exploration of their most favorable niches. For example, buoyant alginate particles can be used for oral drug delivery and its prolonged release in the gastrointestinal tract without the need of precise size control, and sustained release of therapeutic proteins from alginate particles may be achieved without the need for covalent crosslinking.

\section{References}

1. In: Se-Kwon Kim (Eds.), Springer Handbook of Marine Biotechnology. Springer-Verlag Berlin Heidelberg.
2. Papajová E, Bujdoš M, Chorvát D, Stach M, Lacík I (2012) Method for preparation of planar alginate hydrogels by external gelling using an aerosol of gelling solution. Carbohydrate Polymers 90: 472-482.

3. Mazur K, Buchner R, Bonn M, and Hunger J (2014) Hydration of Sodium Alginate in Aqueous Solution. Macromolecules 47: 771-776.

4. Shantha Kumar GS, Shivakumar HG, Pramod kumar TM, Yogananda R, Narayana Charyulu R (2010) Formulation and In-Vitro Evaluation of Buccoadhesive Compacts of Salbutamol Sulphate. International Journal of Drug Development \& Research 2(4): 912-920.

5. De A, Chakraborty S, Mukherjee A, Chattopadhyay J (2013) Formulation of the In-Situ Forming Mucoadhesive Nasal Gel of Salbutamol Using Pluronic F-127 as Thermo sensative Gel Forming Polymer. International Journal of Research in Pharmaceutical and Biomedical Sciences 4(3): 926-933.

6. Jekku N, Nagaraja TS, Yogananda R, Snehalatha (2012) Design and characterisation of salbutamol sulphate mucoadhesive buccal patches. International Journal of Institutional Pharmacy and Life Sciences 2(4): 14-21.

7. Ghorab D, Refai H, Tag R (2011) Preparation and evaluation of fenoterol hydrobromide suppositories. Drug Discoveries \& Therapeutics 5(6): 311-318.

8. Prasanthi NL, Sowmya Krishna C, Eswar Gupta M, Manikiran SS, Rama Rao N (2011) Design and Development of Sublingual Fast Dissolving Films for an Antiasthmatic Drug. Der Pharmacia Lettre 3(1): 382-395.

9. Purushothaman M, Dhanapal R, Vijayakumar S, Vijaya Ratna J (2012) Development of sustained release matrix tablets of salbutamol sulphate using different polymers. International Journal of Advanced Pharmaceutics 2(1): 5-8.

10. Nouh AT, El Gawad HA, Guda TK (2010) Formulation and bioavailability of controlled release salbutamol sulphate tablets using natural additives. Drug Discoveries \& Therapeutics 4(2): 85-92.

11. Sayed S, Ibrahim HK, Mohamed MI, El-Milligi MF (2013) Fast-Dissolving Sublingual Films of Terbutaline Sulfate: Formulation and In Vitro/In Vivo Evaluation. Molecular Pharmaceutics 10: 2942-2947.

12. Qureshi MJ, Ali J, Baboota S, Ahuja A, Mallikarjun C (2014) Pharmacokinetic Study of a Capsule-based Chrono modulated Drug Delivery System of Salbutamol Sulphate in Rabbits. Tropical Journal of Pharmaceutical Research 13(1): 17-22.

13. Kharade S, Bhutkar MA (2013) Novel super disintegrants interpolymeric chitosan-alginate complex and chitin in the formulation of orodispersible tablets. International Journal of Pharmaceutical Research and Development 5(05): 87-94.

14. Pendekal MS, Tegginamat PK (2013) Hybrid drug delivery system for oropharyngeal, cervical and colorectal cancer - in vitro and in vivo evaluation. Saudi Pharmaceutical Journal 21: 177-186.

15. Torsdottir I, Alpsten M, Holm G, Sandberg A-S, Tölli J (1991) A Small Dose of Soluble Alginate-Fiber Affects Postprandial Glycemia and Gastric Emptying in Humans with Diabetes. The Journal of Nutrition 121: 795799.

16. Yamamoto A, Itoh T, Nasu R, Nishida R (2014) Sodium alginate ameliorates indomethacin-induced gastrointestinal mucosal injury via inhibiting translocation in rats. World Journal of Gastroenterology 20(10): 2641-2652.

17. Mirshafiey A, Khodadadi A, Rehm BH, Khorramizadeh MR, Eslami MB, et al. (2005) Sodium Alginate as a Novel Therapeutic Option in Experimental Colitis. Scandinavian Journal of Immunology 61: 316-321.

18. Razavi A, Khodadadi A, Eslami MB, Eshraghi S, Mirshafiey A (2008) Therapeutic Effect of Sodium Alginate in Experimental Chronic Ulcerative Colitis. Iranian Journal of Allergy, Asthma and Immunology $7(1): 13-18$ 
19. Bang MA, Seo JH, Seo JW, Jo GH, Jung SK, et al. (2015) Bacillus subtilis KCTC 11782BP-Produced Alginate Oligosaccharide Effectively Suppresses Asthma via T-Helper Cell Type 2-Related Cytokines. PloS ONE 10(2): e0117524.

20. Waldron-Edward D (1968) Studies on the Inhibition of Intestinal Absorption of Radioactive Strontium: VIII. The Effect of AlginateContaining Diets on Water Metabolism. Canad Med Ass J 99: 986-992.

21. Létourneau V, Nehmé B, Mériaux A, Massé D, Cormier Y, et al. (2010) Human pathogens and tetracycline-resistant bacteria in bioaerosols of swine confinement buildings and in nasal flora of hog producers. International Journal of Hygiene and Environmental Health 213: 444449.

22. Kashef N, Behzadian Nejad Q Najar Peerayeh S, Mousavi Hosseini K Moazzeni M, et al. (2006) Synthesis and characterization of Pseudomonas aeruginosa alginate-tetanus toxoid conjugate. Journal of Medical Microbiology 55: 1441-1446.

23. Xie K, Bai RZ, Wu Y, Liu Q, Liu K, et al. (2009) Anti-tumor effects of a human VEGFR-2-based DNA vaccine in mouse models. Genetic Vaccines and Therapy 7: 10

24. Cheng W, Liu CH, Kuo CM, Chen J-C (2005) Dietary administration of sodium alginate enhances the immune ability of white shrimp Litopenaeus vannamei and its resistance against Vibrio alginolyticus. Fish \& Shellfish Immunology 18: 1-12.

25. Xu X, Iwamoto Y, Kitamura Y, Oda T, Muramatsu T (2003) Root Growthpromoting Activity of Unsaturated Oligomeric Uronates from Alginate on Carrot and Rice Plants. Bioscience, Biotechnology, and Biochemistry 67(9): 2022-2025.

26. Iwasaki K, Matsubara Y (2000) Purification of Alginate Oligosaccharides with Root-Growth-promoting Activity toward Lettuce. Biosci Biotechnol Biochem 64(5): 1067-1070.

27. Natsume M, Kamo Y, Hirayama M, Adachi T (1994) Isolation and characterization of alginate-derived oligosaccharides with root growthpromoting activities. Carbohydrate Research 258: 187-197.

28. Tomoda Y, Umemura K, Adachi T (1994) Promotion of Barley Root Elongation under Hypoxic Conditions by Alginate Lyase-Lysate (A.L.L.). Biosci Biotech Biochem 58(1): 202-203.

29. Yonemoto Y, Tanaka H, Yamashita T, Kitabatake N, Ishida Y, et al. (1993) Promotion of Germination and Shoot Elongation of Some Plants by Alginate Oligomers Prepared with Bacterial Alginate Lyase. Journal of Fermentation and Bioengineering 75(1): 68-70.

30. Zhang Y, Yin H, Zhao X, Wang W, Du Y, et al. (2014) The promotive effects of alginate oligosaccharides on root development in Oryza sativa L. mediated by auxin signaling. Carbohydrate Polymers 113: 446-454.

31. Alam SM, Raza S (2001) Micronutrient Fertilizers. Pakistan Journal of Biological Sciences 4(11): 1446-1450.

32. Mørch ÝA, Sandvig I, Olsen Ø, Donati I, Thuen M, et al. (2012) Mn-alginate gels as a novel system for controlled release of $\mathrm{Mn}^{2+}$ in manganeseenhanced MRI. Contrast Media \& Molecular Imaging 7(2): 265-275.

33. Emmerichs N, Wingender J, Flemming H-C, Mayer C (2004) Interaction between alginates and manganese cations: identification of preferred cation binding sites. International Journal of Biological Macromolecules 34: 73-79.

34. Klinkajon W, Supaphol P (2014) Novel copper (II) alginate hydrogels and their potential for use as anti-bacterial wound dressings. Biomedical Materials 9: 045008.

35. Pavelková M, Kubová K, Vysloužil J, Kejdušová M, Vetchý D, et al. (2017) Biological Effects of Drug-Free Alginate Beads Cross-Linked by Copper Ions Prepared Using External Ionotropic Gelation. AAPS PharmSciTech 18(4): 1343-1354.

36. Straccia MC, Gomez d'Ayala G, Romano I, Laurienzo P (2015) Novel zinc alginate hydrogels prepared by internal setting method with intrinsic antibacterial activity. Carbohydrate Polymers 125: 103-112.
37. Nayak D, Lahiri S (2009) Immobilisation of no-carrier-added ${ }^{93 \mathrm{~m} M o}$ on a biopolymer calcium alginate: a candidate radiopharmaceutical. J Radioanal Nucl Chem 281: 181-183.

38. Veglio' F, Esposito A, Reverberi AP (2002) Copper adsorption on calcium alginate beads: equilibrium $\mathrm{pH}$-related models. Hydrometallurgy 65: 43 57.

39. Concha-Guerrero SI, Brito EMS, Caretta CA (2017) Impact of the Nanomaterials on Soil Bacterial Biodiversity. In: Prasad R, Kumar V, Kumar M (Eds.), Nanotechnology. Singapore.

40. Maddhinni VL, Vurimidi HB, Yerramilli A (2006) Degradation of azo dye with horse radish peroxidase (HRP). J Indian Inst Sci 86: 507-514.

41. Alemzadeh I, Nejati S, Vossoughi M (2009) Removal of Phenols from Wastewater with Encapsulated Horseradish Peroxidase in Calcium Alginate. Engineering Letters 17: 4

42. Bhushan B, Pal A, and Jain V (2015) Improved Enzyme Catalytic Characteristics upon Glutaraldehyde Cross-Linking of Alginate Entrapped Xylanase Isolated from Aspergillus flavus MTCC 9390. Enzyme Research.

43. Lamboley L, St Gelais D, Champagne SD, Lamoureux M (2003) Growth and morphology of thermophilic dairy starters in alginate beads. J Gen Appl Microbiol 49: 205-214.

44. Kumar P, Satyanarayana T (2007) Economical glucoamylase production by alginate-immobilized Thermomucor indicae-seudaticae in cane molasses medium. Letters in Applied Microbiology 45: 392-397.

45. Pawar SN, Edgar KJ (2012) Alginate derivatization: A review of chemistry, properties and applications. Biomaterials 33: 3279-3305.

46. Abouelmagd SA, Sun B, Chang AC, Ku YJ, Yeo Y (2015) Release Kinetics Study of Poorly Water-Soluble Drugs from Nanoparticles: Are We Doing It Right? Molecular Pharmaceutics 12: 997-1003.

47. Marcato PD, Adami LF, Melo PS, de Paula LB, Durán N, et al. AB (2011) Glutathione and S-nitrosoglutathione in alginate/chitosan nanoparticles: Cytotoxicity. Journal of Physics: Conference Series 304: 012045.

48. Marcato PD, Adami LF, de Melo Barbosa R, Melo PS, Ferreira IR, et al. (2013) Development of a Sustained-release System for Nitric Oxide Delivery using Alginate/Chitosan Nanoparticles. Current Nanoscience $9(1)$.

49. Cardozo VF, Lancheros CAC, Narciso AM, Valereto ECS, Kobayashi RKT, et al. (2014) Evaluation of antibacterial activity of nitric oxide-releasing polymeric particles against Staphylococcus aureus and Escherichia coli from bovine mastitis. International Journal of Pharmaceutics 473: 2029.

50. Nellore J, Baskaran T, Kumari A, Namasivayam SKR (2014) Comparative Evaluation of the Antioxidant and Antibacterial Efficacy of Unencapsulated and Encapsulated Essential Oil of Lemongrass in Alginate / Chitosan Microspheres. Journal of Pure and Applied Microbiology 8(4): 2893-2902.

51. Bagre AP, Jain K, Jain NK (2013) Alginate coated chitosan core shell nanoparticles for oral delivery of enoxaparin: In vitro and in vivo assessment. International Journal of Pharmaceutics 456: 31-40.

52. Singh R, Lillard Jr JW (2009) Nanoparticle-based targeted drug delivery. Experimental and Molecular Pathology 86: 215-223.

53. Grekhnyova EV, Kudryavtseva TN (2014) Characteristics of microencapsulation some drugs in sodium alginate. Auditorium 3(3).

54. Grekhnyova EV, Kudryavtseva TN, Klimova LG (2017) Properties furatsilina encapsulated in a water-soluble polymers. Auditorium 1(13).

55. Bagheri L, Madadlou A, Yarmand M, Mousavi ME (2014) Spray-dried alginate microparticles carrying caffeine-loaded and potentially bioactive nanoparticles. Food Research International 62: 1113-1119.

56. De Cicco F, Porta A, Sansone F, Aquino RP, Del Gaudio P (2014) Nanospray technology for an in situ gelling nanoparticulate powder as a wound dressing. International Journal of Pharmaceutics 473: 30-37. 
57. Li Q Liu CG, Huang ZH, Xue FF (2011) Preparation and Characterization of Nanoparticles Based on Hydrophobic Alginate Derivative as Carriers for Sustained Release of Vitamin $\mathrm{D}_{3}$. Journal of Agricultural and Food Chemistry 59: 1962-1967.

58. Schütz CA, Juillerat-Jeanneret L, Käuper P, Wandrey C (2011) Cell Response to the Exposure to Chitosan-TPP//Alginate Nanogels. Biomacromolecules 12: 4153-4161.

59. Wen Y, Grøndahl L, Gallego MR, Jorgensen L, Møller EH, et al. (2012) Delivery of Dermatan Sulfate from Polyelectrolyte ComplexContaining Alginate Composite Microspheres for Tissue Regeneration. Biomacromolecules 13: 905-917.

60. Zhang W, He S, Liu Y, Geng Q, Ding G, et al. (2014) Preparation and Characterization of Novel Functionalized Prochloraz Microcapsules Using Silica-Alginate-Elements as Controlled Release Carrier Materials. ACS Applied Materials \& Interfaces 6: 11783-11790.

61. Yurkova IN, Omelchenko AV, Bugara IA (2014) The influence of silver nanoparticles on wheat growth process. ESSUTM Bulletin 1: 69-73.

62. Leopold N, Chiş V, Mircescu NE, Marișca OT, Buja OM, et al. (2013) One step synthesis of SERS active colloidal gold nanoparticles by reduction with polyethylene glycol. Colloids and Surfaces A: Physicochemical and Engineering Aspects 436: 133-138.

63. Pestovsky Y (2017) Calcium Alginate Nanoparticles with Adjustable Size for Prolonged Glutathione Release. Proceedings of BIT's World Gene Convention-2017. Macau, China.

64. Stanislavov AS, Yanovska AA, Kuznetsov VN, Sukhodub LB, Sukhodub LF (2015) The Comparison of Magnetite Nanospheres Formation in Polysaccharide Covers by Various Ways of Syntheses. Journal of Nanoand Electronic Physics 7(2): 02009.

65. Zhao D, Liu CJ, Zhuo RX, Cheng SX (2012) Alginate $/ \mathrm{CaCO}_{3}$ Hybrid Nanoparticles for Efficient Codelivery of Antitumor Gene and Drug. Molecular Pharmaceutics 9: 2887-2893.

66. Shutava TG (2013) Nano- and microcontainers from biopolymers and biologically active compounds. Proceedings of the National Academy of Sciences of Belarus. Chemical series 4: 51-61.

67. Liu Q Rauth AM, Wu XY (2007) Immobilization and bioactivity of glucose oxidase in hydrogel microspheres formulated by an emulsificationinternal gelation-adsorption-polyelectrolyte coating method. International Journal of Pharmaceutics 339: 148-156.

68. Poncelet D (2001) Production of Alginate Beads by Emulsification/ Internal Gelation. Annals of the New York Academy of Sciences 944(1): 74-82.

69. Hoesli CA, Raghuram K, Kiang RLJ, Mocinecová D, Hu X, et al. (2011) Pancreatic Cell Immobilization in Alginate Beads Produced by Emulsion and Internal Gelation. Biotechnology and Bioengineering 108(2): 424434.

70. Bharathi B, Sirisha B, Maheshwara rao VU, Vijaya lakshmi P, Ajitha M, et al. (2014) Development and Characterisation of Salbutamol Sulphate Hydrogel Beads by Using Emulsion Internal Ionotropic Gelation Technique. International Journal of Pharma Research and Health Sciences 2(6): 447-456.

71. Mudhakir D, Bostanudin MF, Firmawan F, Mauludin R (2012) Design of Salbutamol Sulphate Gastroretentive Nanoparticles via Surface Charge Manipulation. International Scholarly and Scientific Research \& Innovation 6(12): 65-71.

72. Reis CP, Ribeiro AJ, Neufeld RJ, Veiga F (2007) Alginate Microparticles as Novel Carrier for Oral Insulin Delivery. Biotechnology and Bioengineering 96(5): 977-989.

73. Lopes M, Shrestha N, Correia A, Shahbazi MA, Sarmento B, et al. (2016) Dual chitosan/albumin-coated alginate/dextran sulfate nanoparticles for enhanced oral delivery of insulin. Journal of Controlled Release 232: 29-41.
74. Zhao Y, Carvajal MT, Won YY, Harris MT (2007) Preparation of Calcium Alginate Microgel Beads in an Electro dispersion Reactor Using an Internal Source of Calcium Carbonate Nanoparticles. Langmuir 23(25): 12489-12496

75. Chueh B, Zheng Y, Torisawa Y, Hsiao AY, Ge C, et al. (2010) Patterning alginate hydrogels using light-directed release of caged calcium in a microfluidic device. Biomed Microdevices 12(1): 145-151.

76. Reis CP, Neufeld RJ, Vilela S, Ribeiro AJ, Veiga F (2006) Review and current status of emulsion/dispersion technology using an internal gelation process for the design of alginate particles. Journal of Microencapsulation 23(3): 245-257.

77. Paques JP, van der Linden E, van Rijn CJM, Sagis LMC (2014) Preparation methods of alginate nanoparticles. Advances in Colloid and Interface Science 209: 163-171.

78. Pechenkina AN, Saprina VI, Lovskaya DD, Menshutina NV (2015) The usage of polysaccharide aerogels as modern drug delivery systems. Advances in chemistry and chemical technology 29(4): 48-50.

79. Masalova OA, Shutava TG (2015) The effect of stabilizers on properties of calcium alginate nanoparticles. Proceedings of the National Academy of Sciences of Belarus. Chemical series 1: 47-51.

80. You JO, Peng CA (2005) Calcium-Alginate Nanoparticles Formed by Reverse Microemulsion as Gene Carriers. Macromolecular Symposia 219: $147-153$

81. Hori Y, Winans AM, Huang CC, Horrigan EM, Irvine DJ (2008) Injectable dendritic cell-carrying alginate gels for immunization and immunotherapy. Biomaterials 29: 3671-3682.

82. Hori Y, Stern P, Hynes RO, Irvine DJ (2009) Engulfing tumors with synthetic extracellular matrices for cancer immunotherapy. Biomaterials 30(35): 6757-6767.

83. Mugli M, Hiremath D, Patil A (2012) Studies on Microencapsulation of Salbutamol Sulphate. Research Journal of Pharmaceutical, Biological and Chemical Sciences 3(2): 112-118.

84. Malakar J, Datta PK, Purakayastha SD, Dey S, Nayak AK (2014) Floating capsules containing alginate-based beads of salbutamol sulfate: In vitroin vivo evaluations. International Journal of Biological Macromolecules 64: 181-189.

85. Machado AHE, Lundberg D, Ribeiro AJ, Veiga FJ, Lindman B, et al. (2012) Preparation of Calcium Alginate Nanoparticles Using Water-in-Oil (W/0) Nanoemulsions. Langmuir 28: 4131-4141.

86. Nesamony J, Singh PR, Nada SE, Shah ZA, Kolling WM (2012) Calcium Alginate Nanoparticles Synthesized Through a Novel Interfacial CrossLinking Method as a Potential Protein Drug Delivery System. Journal of Pharmaceutical Sciences 101(6): 2177-2184.

87. Yas A (2013) Preparation and characterization of in situ cross-linking alginate/polyethylene glycol 6000 blend microparticles for controlling salbutamol sulphate pulmonary delivery. International Journal of Pharmacy and Pharmaceutical Sciences 5(4): 728-733.

88. Prasanth VV, Chakraborty A, Mathew ST, Parthasarathy G, Mathappan $\mathrm{R}$, et al. (2011) Formulation and evaluation of salbutamol sulphatealginate microspheres by ionotropic gelation method. Pharmacie Globale 02(07): 1-4.

89. Huang KS, Lai TH, Lin YC (2006) Manipulating the generation of Caalginate microspheres using microfluidic channels as a carrier of gold nanoparticles. Lab on a Chip 6: 954-957.

90. Wang Q Qian K, Liu S, Yang Y, Liang B, et al. (2015) X-ray Visible and Uniform Alginate Microspheres Loaded with in Situ Synthesized $\mathrm{BaSO}_{4}$ Nanoparticles for in Vivo Transcatheter Arterial Embolization. Biomacromolecules 16(4): 1240-1246.

91. Chavanpatil MD, Khdair A, Gerard B, Bachmeier C, Miller DW, et al. (2007) Surfactant-Polymer Nanoparticles Overcome P-GlycoproteinMediated Drug Efflux. Molecular Pharmaceutics 4(5): 730-738. 
92. Khdair A, Gerard B, Handa H, Mao G, Shekhar MPV, et al. (2008) Surfactant-Polymer Nanoparticles Enhance the Effectiveness of Anticancer Photodynamic Therapy. Molecular Pharmaceutics 5(5): 795807.

93. Usacheva M, Swaminathan SK, Kirtane AR, Panyam J (2014) Enhanced Photodynamic Therapy and Effective Elimination of Cancer Stem Cells Using Surfactant-Polymer Nanoparticles. Molecular Pharmaceutics 11: 3186-3195.

94. Khdair A, Chen D, Patil Y, Ma L, Dou QP, et al. (2010) Nanoparticlemediated combination chemotherapy and photodynamic therapy overcomes tumor drug resistance. J Control Release 141(2): 137-144.

95. Chavanpatil MD, Khdair A, Patil Y, Handa H, Mao G, et al. (2007) PolymerSurfactant Nanoparticles for Sustained Release of Water-Soluble Drugs. Journal of Pharmaceutical Sciences 96(12): 3379-3389.

96. Kumar S, Bhanjana G, Sharma A, Sidhu MC, Dilbaghi N (2014) Synthesis, characterization and on field evaluation of pesticide loaded sodium alginate nanoparticles. Carbohydrate Polymers 101: 1061-1067.

97. Masalova O, Kulikouskaya V, Shutava T, Agabekov V (2013) Alginate and chitosan gel nanoparticles for efficient protein entrapment. Physics Procedia 40: 69-75.

98. Mukhopadhyay P, Chakraborty S, Bhattacharya S, Mishra R, Kundu PP (2015) pH-sensitive chitosan/alginate core-shell nanoparticles for efficient and safe oral insulin delivery. International Journal of Biological Macromolecules 72: 640-648.

99. Saraei F, Mohamadpour Dounighi N, Zolfagharian H, Moradi Bidhendi S, Khaki P, et al. (2013) Design and evaluate alginate nanoparticles as a protein delivery system. Archives of Razi Institute 68(2): 139-146.

100. Sarmento B, Ferreira D, Veiga F, Ribeiro A (2006) Characterization of insulin-loaded alginate nanoparticles produced by ionotropic pregelation through DSC and FTIR studies. Carbohydrate Polymers 66 $1-7$.

101. Valieva LV, Krasnoshtanova AA (2017) Development of the oral delivery system of prolonged insulin by chitosan-alginate nanoparticles. Advances in chemistry and chemical technology 31(9): 23-25.

102. Kumar S, Chauhan N, Gopal M, Kumar R, Dilbaghi N (2015) Development and evaluation of alginate-chitosan nanocapsules for controlled release of acetamiprid. International Journal of Biological Macromolecules 81: 631-637.

103. Silva MdS, Sgarbi Cocenza D, Grillo R, Silva de Melo NF, Tonello PS, et al (2011) Paraquat-loaded alginate/chitosan nanoparticles: Preparation, characterization and soil sorption studies. Journal of Hazardous Materials 190: 366-374.

104. Rodrigues Maruyama C, Guilger M, Pascoli M, Bileshy-José N, Abhilash PC, et al. (2016) Nanoparticles Based on Chitosan as Carriers for the Combined Herbicides Imazapic and Imazapyr. Scientific Reports 6: 19768.

105. Ahmad Z, Pandey R, Sharma S, Khuller GK (2006) Alginate Nanoparticles as Anti tuberculosis Drug Carriers: Formulation Development, Pharmacokinetics and Therapeutic Potential. The Indian Journal of Chest Diseases \& Allied Sciences 48: 171-176.

106. Ahmad Z, Sharma S, Khuller GK (2007) Chemotherapeutic evaluation of alginate nanoparticle-encapsulated azole antifungal and antitubercular drugs against murine tuberculosis. Nanomedicine: Nanotechnology, Biology, and Medicine 3: 239-243.

107. Zahoor A, Sharma S, Khuller GK (2005) Inhalable alginate nanoparticles as antitubercular drug carriers against experimental tuberculosis International Journal of Antimicrobial Agents 26: 298-303.

108. Menon JU, Ravikumar P, Pise A, Gyawali D, Hsia CCW, et al. (2014) Polymeric nanoparticles for pulmonary protein and DNA delivery. Acta Biomaterialia 10: 2643-2652.

109. Li T, Shi XW, Du YM, Tang YF (2007) Quaternized chitosan/alginate nanoparticles for protein delivery. Journal of Biomedical Materials Research Part A 83(2): 383-390.
110. Samimi M, Alimova FK, Abtahi B, Validov SZ (2015) Characteristics and transfection efficacy of DNA-loaded biocomposite nanoparticles. Modern problems of science and education 3.

111. Nigam SC, Tsao IF, Sakoda A, Wang HY (1988) Techniques for preparing hydrogel membrane capsules. Biotechnology Techniques 2(4): 271276.

112. Batyrbekov EO, Rahimbaeva DZ, Musabekov KB, Zhubanov BA (2009) Prolonged cyclophosphamide release from alginate microparticles. Izvestiia Natsional'noi Akademii nauk Respubliki Kazakhstan. Seriia khimicheskaia 4: 22-25.

113. Quintanilla Guerrero F, Duarte Vázquez MA, García Almendarez BE Tinoco R, Vazquez Duhalt R, et al. (2008) Polyethylene glycol improves phenol removal by immobilized turnip peroxidase. Bioresource Technology 99: 8605-8611.

114. Quong D, Neufeld RJ, Skjåk Bræk G, Poncelet D (1998) External Versus Internal Source of Calcium During the Gelation of Alginate Beads for DNA Encapsulation. Biotechnology and Bioengineering 57(4): 438446.

115. Sivasubramanian S, Namasivayam SKR (2014) Optimization of parameters for phenol degradation using immobilized Candida tropicalis SSK01 in batch reactor. Journal of Environmental Biology 35: 531-536.

116. Moshaverinia A, Ansari S, Chen C, Xu X, Akiyama K, et al. (2013) Coencapsulation of anti-BMP2 monoclonal antibody and mesenchymal stem cells in alginate microspheres for bone tissue engineering. Biomaterials 34: 6572-6579.

117. Gonzalez LE, Bashan Y (2000) Increased Growth of the Microalga Chlorella vulgaris when Coimmobilized and Cocultured in Alginate Beads with the Plant-Growth-Promoting Bacterium Azospirillum brasilense. Applied and Environmental Microbiology 66(4): 1527 1531.

118. Cook MT, Tzortzis G, Charalampopoulos D, Khutoryanskiy VV (2011) Production and Evaluation of Dry Alginate-Chitosan Microcapsules as an Enteric Delivery Vehicle for Probiotic Bacteria. Biomacromolecules 12: $2834-2840$

119. Bakhytkyzy I, Musabekov KB (2015) Calcium alginate microparticles containing anti-TB drugs. Chemical Bulletin of Kazakh National University 2(78): 14-20.

120. Pivnenko TN, Buntush AE (2017) Experimental foundation of microcapsulation of oil extract of carothinoids from tunics of purple ascidian. Scientific Journal of DALRYBVTUZ 42: 82-89.

121. Ouyang W, Chen H, Jones ML, Metz T, Haque T, et al. (2004) Artificial cell microcapsule for oral delivery of live bacterial cells for therapy: design, preparation, and in-vitro characterization. J Pharm Pharmaceut Sci $7(3): 315-324$

122. Wideman RD, Gray SL, Covey SD, Webb GC, Kieffer TJ (2009) Transplantation of PC1/3-Expressing $\alpha$-cells Improves Glucose Handling and Cold Tolerance in Leptin-resistant Mice. Molecular Therapy 17(1): 191-198.

123. Tsoy AM, Zaytseva Zotova DS, Edelweiss EF, Bartkowiak A, Goergen JL, et al. (2010) Microencapsulated multicellular tumor spheroids: preparation and use as a novel in vitro model for drug screening. Biomeditsinskaya Khimiya 56(6): 674-685.

124. Indhumathi D, Varma M, Reddy NRC, Nikhil P, Anandaraj P (2014) Formulation and optimization of salbutamol sulphate enclosed in alginate microbeads prepared by ionotropic gelation method. World Journal of Pharmacy and Pharmaceutical Sciences 3(9): 642-653.

125. Singh B, Purohit S, Saini G, Bhandari A, Awasthi D (2013) Colon specific chronotherapeutic drug delivery for nocturnal asthma: Eudragit S-100 coated calcium alginate gel beads-entrapped salbutamol sulphate. International Journal of Pharmacy and Pharmaceutical Sciences 5(2): 362-367. 
126. Wang X, Wenk E, Hu X, Castro GR, Meinel L, et al. (2007) Silk coatings on PLGA and alginate microspheres for protein delivery. Biomaterials 28: 4161-4169.

127. Champagne CP, Blahuta N, Brion F, Cagnon C (2000) A Vortex-Bowl Disk Atomizer System for the Production of Alginate Beads in a 1500-Liter Fermentor. Biotechnology and Bioengineering 68(6): 682-688.

128. Li P, Dai YN, Zhang JP, Wang AQ Wei Q (2008) Chitosan-Alginate Nanoparticles as a Novel Drug Delivery System for Nifedipine. International Journal of Biomedical Science 4(3): 221-228.

129. Das RK, Kasoju N, Bora U (2010) Encapsulation of curcumin in alginatechitosan-pluronic composite nanoparticles for delivery to cancer cells. Nanomedicine: Nanotechnology, Biology, and Medicine 6: 153-160.

130. Choi BY, Park HJ, Hwang SJ, Park JB (2002) Preparation of alginate beads for floating drug delivery system: effects of $\mathrm{CO}_{2}$ gas-forming agents. International Journal of Pharmaceutics 239: 81-91.

131. Quong D, Neufeld RJ (1998) DNA Protection from Extracapsular Nucleases, within Chitosan- or Poly-L-lysine-Coated Alginate Beads. Biotechnology and Bioengineering 60(1): 124-134.

132. El Sherbiny IM, Smyth HDC (2011) Smart Magnetically Responsive Hydrogel Nanoparticles Prepared by a Novel Aerosol-Assisted Method for Biomedical and Drug Delivery Applications. Journal of Nanomaterials.

133. Guzman Villanueva D, Smyth HDC, Herrera Ruiz D, El Sherbiny IM (2011) A Novel Aerosol Method for the Production of Hydrogel Particles. Journal of Nanomaterials.

134. Aliseda A, Hopfinger EJ, Lasheras JC, Kremer DM, Berchielli A, et al. (2008) Atomization of viscous and non-newtonian liquids by a coaxial, high-speed gas jet. Experiments and droplet size modeling. International Journal of Multiphase Flow 34: 161-175.

135. Naz MY, Sulaiman SA, Ariwahjoedi B (2015) Effect of the borax mass and pre-spray medium temperature on droplet size and velocity vector distributions of intermittently sprayed starchy solutions. Phys Chem Chem Phys 17: 3704-3714.

136. Movahednejad E, Ommi F, Hosseinalipour SM (2010) Prediction of Droplet Size and Velocity Distribution in Droplet Formation Region of Liquid Spray. Entropy 12: 1484-1498.

137. Pestovsky YS (2013) Horseradish peroxidase immobilization in calcium alginate hydrogel and microparticles. Polythematic online scientific journal of Kuban State Agrarian University 92(8).

138. Cocchietto M, Blasi P, Lapasin R, Moro C, Gallo D, et al. (2013) Microencapsulation of Bioactive Principles with an Airless Spray-Gun Suitable for Processing High Viscous Solutions. Journal of Functional Biomaterials 4: 312-328.

139. Tapia C, Montezuma V, Yazdani Pedram M (2008) Microencapsulation by Spray Coagulation of Diltiazem $\mathrm{HCl}$ in Calcium Alginate-Coated Chitosan. AAPS PharmSciTech 9(4): 1198-1206.

140. Mobed Miremadi M, Asi B, Parasseril J, Wong E, Tat M, et al. (2013) Comparative diffusivity measurements for alginate-based atomized and inkjet-bioprinted artificial cells using fluorescence microscopy. Artificial Cells, Nanomedicine, and Biotechnology 41: 196-201.

141. Duvivier Kali VF, Omer A, Parent RJ, O’Neil JJ, Weir GC (2001) Complete Protection of Islets Against Allorejection and Autoimmunity by a Simple Barium-Alginate Membrane. Diabetes 50: 1698-1705.

142. Schneider S, Feilen PJ, Brunnenmeier F, Minnemann T, Zimmermann $\mathrm{H}$, et al. (2005) Long-Term Graft Function of Adult Rat and Human Islets Encapsulated in Novel Alginate-Based Microcapsules After Transplantation in Immunocompetent Diabetic Mice. Diabetes 54: 687-693.

143. Omer A, Duvivier Kali VF, Trivedi N, Wilmot K, Bonner Weir S, et al. (2003) Survival and Maturation of Microencapsulated Porcine Neonatal Pancreatic Cell Clusters Transplanted into Immunocompetent Diabetic Mice. Diabetes 52: 69-75.
144. Tuch BE, Keogh GW, Williams LJ, Wu W, Foster JL, et al. (2009) Safety and Viability of Microencapsulated Human Islets Transplanted Into Diabetic Humans. Diabetes Care 32(10): 1887-1889.

145. Cheow WS, Hadinoto K (2013) Biofilm-Like Lactobacillus rhamnosus Probiotics Encapsulated in Alginate and Carrageenan Microcapsules Exhibiting Enhanced Thermotolerance and Freeze-Drying Resistance. Biomacromolecules 14: 3214-3222.

146. Liao YT, Liu CH, Yu J, Wu KCW (2014) Liver cancer cells: targeting and prolonged-release drug carriers consisting of mesoporous silica nanoparticles and alginate microspheres. International Journal of Nanomedicine 9: 2767-2778.

147. Tapia Hernández JA, Torres Chávez PI, Ramírez Wong B, Rascón Chu A, Plascencia Jatomea M, et al. (2015) Micro- and Nanoparticles by Electrospray: Advances and Applications in Foods. Journal of Agricultural and Food Chemistry 63: 4699-4707.

148. Smrdel P, Bogataj M, Mrhar A (2008) The Influence of Selected Parameters on the Size and Shape of Alginate Beads Prepared by Ionotropic Gelation. Sci Pharm 76: 77-89.

149. Dessy A, Piras AM, Chiellini F (2013) Hemoglobin Loaded Alginate Particles. J Biomim Biomater Tissue Eng 18(1): 102.

150. Broniarz Press L, Ochowiak M, Włodarczyk S, Markuszewska M (2013) Effervescent atomization of glycerol aqueous solutions. Transactions of the Institute of fluid-flow machinery 125: 29-38.

151. Bhattarai N, Zhang M (2007) Controlled synthesis and structural stability of alginate-based nanofibers. Nanotechnology 18: 455601.

152. Smidsrød O, Haug A (1965) The Effect of Divalent Metals on the Properties of Alginate Solutions. I Calcium Ions Acta Chemica Scandinavica 19(2): 329-340.

153. Smidsrød O, Haug A, Larsen B (1963) Degradation of Alginate in the Presence of Reducing Compounds. Acta Chemica Scandinavica 17: 2628-2637.

154. Yang Z, Li JP, Guan H (2004) Preparation and characterization of oligomannuronates from alginate degraded by hydrogen peroxide. Carbohydrate Polymers 58: 115-121.

155. Gilbert DL, Gerschman R, Cohen J, Sherwood W (1957) The Influence of High Oxygen Pressures on the Viscosity of Solutions of Sodium Desoxyribonucleic Acid and of Sodium Alginate. JACS 79: 5677-5680.

156. Ip AY, Niven RW (1994) Prediction and Experimental Determination of Solute Output from a Collision Nebulizer. Journal of Pharmaceutical Sciences 83(7): 1047-1051.

157. Longest PW, Spence BM, Holbrook LT, Mossi KM, Son YJ, et al. (2012) Production of Inhalable Submicrometer Aerosols from Conventional Mesh Nebulizers for Improved Respiratory Drug Delivery. J Aerosol Sci 51: 66-80.

158. Semião V, Andrade P, Carvalho MG (1996) Spray characterization: numerical prediction of Sauter mean diameter and droplet size distribution. Fuel 75(15): 1707-1714.

159. Kim WT, Mitra SK, Li X, Prociw LA, Hu TCJ (2003) A Predictive Model for the Initial Droplet Size and Velocity Distributions in Sprays and Comparison with Experiments. Part Part Syst Charact 20: 135-149.

160. Babinsky E, Sojka PE (2002) Modeling drop size distributions. Progress in Energy and Combustion Science 28: 303-329.

161. Wu Z, Su X, Xu Y, Kong B, Sun W, et al. (2016) Bioprinting threedimensional cell-laden tissue constructs with controllable degradation. Scientific Reports 6: 24474.

162. Vreeker R, Li L, Fang Y, Appelqvist I, Mendes E (2008) Drying and Rehydration of Calcium Alginate Gels. Food Biophysics 3: 361-369.

163. Szejtli J (1998) Introduction and General Overview of Cyclodextrin Chemistry. Chemical Reviews 98: 1743-1753. 
164. Reineccius TA, Reineccius GA, Peppard TL (2002) Encapsulation of Flavors using Cyclodextrins: Comparison of Flavor Retention in Alpha, Beta, and Gamma Types. Journal of Food Science 67(9): 3271-3279.

165. Gong L, Li T, Chen F, Duan X, Yuan Y, et al. (2016) An inclusion complex of eugenol into $\beta$-cyclodextrin: Preparation, and physicochemical and antifungal characterization. Food Chemistry 196: 324-330.

166. Li X, Jin Z, Wang J (2007) Complexation of allyl isothiocyanate by $\alpha$ and $\beta$-cyclodextrin and its controlled release characteristics. Food Chemistry 103: 461-466.

167. Piercey MJ, Mazzanti G, Budge SM, Delaquis PJ, Paulson AT, et al. (2012) Antimicrobial activity of cyclodextrin entrapped allyl isothiocyanate in a model system and packaged fresh-cut onions. Food Microbiology 30: 213-218.

168. Abarca RL, Rodríguez FJ, Guarda A, Galotto MJ, Bruna JE (2016) Characterization of beta-cyclodextrin inclusion complexes containing an essential oil component. Food Chemistry 196: 968-975.

169. Almenar E, Auras R, Rubino M, Harte B (2007) A new technique to prevent the main post harvest diseases in berries during storage: Inclusion complexes $\beta$-cyclodextrin-hexanal. International Journal of Food Microbiology 118: 164-172.

170. Zi P, Yang X, Kuang H, Yang Y, Yu L (2008) Effect of HP $\beta C D$ on solubility and transdermal delivery of capsaicin through rat skin. Internationa Journal of Pharmaceutics 358: 151-158.

171. Chen X, Sun X, Ren K, Zhang X, Zhang Z, et al. (2010) Enhanced aqueous solubility and bioavailability of capsaicin by the preparation of an inclusion complex. Arzneimittelforschung 60(9): 571-574.

172. Zhao Y, Sun C, Shi F, Firempong CK, Yu J, et al. (2016) Preparation, characterization, and pharmacokinetics study of capsaicin via hydroxypropyl-beta-cyclodextrin encapsulation. Pharmaceutica Biology 54(1): 130-138.

173. Almenar E, Auras R, Wharton P, Rubino M, Harte B (2007) Release of Acetaldehyde from $\beta$-Cyclodextrins Inhibits Postharvest Decay Fungi in Vitro. Journal of Agricultural and Food Chemistry 55(17): 72057212 .

174. Szente L, Szejtli J (1995) Cyclodextrin-Complexed Acetal as an Acetaldehyde Generator. Perfumer \& Flavorist 20:11-13.

175. Celebioglu A, Sen HS, Durgun E, Uyar T (2016) Molecular entrapment of volatile organic compounds (VOCs) by electrospun cyclodextrin nanofibers. Chemosphere 144: 736-744.

176. Thompson D (2007) In Silico Engineering of Tailored Ink-Binding Ability at Molecular Printboards. ChemPhysChem 8: 1684-1693.

177. Hill LE, Gomes C, Taylor TM (2013) Characterization of betacyclodextrin inclusion complexes containing essential oils (transcinnamaldehyde, eugenol, cinnamon bark, and clove bud extracts) for antimicrobial delivery applications. LWT - Food Science and Technology 51: 86-93.

178. Cabral Marques HM (2010) A review on cyclodextrin encapsulation of essential oils and volatiles. Flavour and Fragrance Journal 25: 313-326.

179. Huang L, Xin J, Guo Y, Li J (2010) A Novel Insulin Oral Delivery System Assisted by Cationic $\beta$-Cyclodextrin Polymers. Journal of Applied Polymer Science 115: 1371-1379.

180. Zhang N, Li J, Jiang W, Ren C, Li J, et al. (2010) Effective protection and controlled release of insulin by cationic $\beta$-cyclodextrin polymers from alginate/chitosan nanoparticles. International Journal of Pharmaceutics 393: 212-218.

181. Mansourpour M, Mahjub R, Amini M, Ostad SN, Shamsa ES, et al. (2015) Development of Acid-Resistant Alginate/Trimethyl Chitosan Nanoparticles Containing Cationic $\beta$-Cyclodextrin Polymers for Insulin Oral Delivery. AAPS PharmSciTech 16(4): 952-962.

182. Miao T, Fenn SL, Charron PN, Oldinski RA (2015) Self-Healing and Thermoresponsive Dual-Cross-Linked Alginate Hydrogels Based on
Supramolecular Inclusion Complexes. Biomacromolecules 16: 3740 3750.

183. Santagapita PR, Mazzobre MF, Buera MP (2011) Formulation and Drying of Alginate Beads for Controlled Release and Stabilization of Invertase. Biomacromolecules 12: 3147-3155.

184. Amaro MI, Tajber L, Corrigan OI, Healy AM (2015) Co-Spray Dried Carbohydrate Microparticles: Crystallisation Delay/Inhibition and Improved Aerosolization Characteristics Through the Incorporation of Hydroxypropyl- $\beta$-cyclodextrin with Amorphous Raffinose or Trehalose. Pharm Res 32: 180-195.

185. Bornaghi L, Utille JP, Penninga D, Schmidt AK, Dijkhuizen L, etal. (1996) Enzymic synthesis of cyclothiomaltins. Chemical Communications, pp. 2541-2542.

186. Excoffier G, Paillet M, Vignon M (1985) Cyclic ( $1 \rightarrow 6)-\beta$-D-glucopyranose oligomers: synthesis of cyclogentiotriose and cyclogentiotetraose peracetates. Carbohydrate Research 135: C10-C11.

187. Morales JC, Zurita D, Penadés S (1998) Carbohydrate-Carbohydrate Interactions in Water with Glycophanes as Model Systems. J Org Chem 63(25): 9212-9222.

188. Li W-W, Claridge TDW, Li Q, Wormald MR, Davis BG, et al. (2011) Tuning the Cavity of Cyclodextrins: Altered Sugar Adaptors in Protein Pores. JACS 133: 1987-2001.

189. Kida T, Kikuzawa A, Nakatsuji Y, Akashi M (2003) A facile synthesis of novel cyclodextrin derivatives incorporating one $\beta-(1,4)$-glucosidic bond and their unique inclusion ability. Chemical Communications, pp. 3020-3021.

190. Kikuzawa A, Kida T, Nakatsuji Y, Akashi M (2005) Short Synthesis of Skeleton-Modified Cyclodextrin Derivatives with Unique Inclusion Ability. J Org Chem 70: 1253-1261.

191. Hoffmann B, Bernet B, Vasella A (2002) Oligosaccharide Analogues of Polysaccharides. Part 24) Synthesis of Cyclodextrin Analogues Containing a Substituted Buta-1,3-diyne or a 1,2,3-Triazole Unit and Analysis of Intramolecular Hydrogen Bonds. Helvetica Chimica Acta 85: 265-287.

192. Yang B, Dong X, Lei Q Zhuo R, Feng J, et al. (2015) Host-Guest Interaction-Based Self-Engineering of Nano-Sized Vesicles for CoDelivery of Genes and Anticancer Drugs. ACS Applied Materials \& Interfaces 7: 22084-22094.

193. Andersen T, Auk-Emblem P, Dornish M (2015) 3D Cell Culture in Alginate Hydrogels. Microarrays 4: 133-161.

194. Schmid T, Burkhard J, Yeo B-S, Zhang W, Zenobi R (2008) Towards chemical analysis of nanostructures in biofilms I: imaging of biological nanostructures. Analytical and Bioanalytical Chemistry 391: 18991905.

195. Simpliciano C, Clark L, Asi B, Chu N, Mercado M, et al. (2013) CrossLinked Alginate Film Pore Size Determination Using Atomic Force Microscopy and Validation Using Diffusivity Determinations. Journal of Surface Engineered Materials and Advanced Technology 3: 1-12.

196. Hannoun BJM, Stephanopoulos G (1986) Diffusion Coefficients of Glucose and Ethanol in Cell-free and Cell-occupied Calcium Alginate Membranes. Biotechnology and Bioengineering 28: 829-835.

197. Kulkarni AR, Soppimath KS, Aminabhavi TM (1999) Controlled release of diclofenac sodium from sodium alginate beads crosslinked with glutaraldehyde. Pharmaceutica Acta Helvetiae 74: 29-36.

198. Kulkarni AR, Soppimath KS, Aminabhavi TM, Dave AM, Mehta MH (2000) Glutaraldehyde crosslinked sodium alginate beads containing liquid pesticide for soil application. Journal of Controlled Release 63: 97-105.

199. Teli SB, Gokavi GS, Sairam M, Aminabhavi TM (2007) Highly water selective silicotungstic acid $\left(\mathrm{H}_{4} \mathrm{SiW}_{12} \mathrm{O}_{40}\right)$ incorporated novel sodium alginate hybrid composite membranes for pervaporation dehydration of acetic acid. Separation and Purification Technology 54: 178-186. 
200. Bhat SD, Mallikarjuna NN, Aminabhavi TM (2006) Microporous alumino-phosphate $\left(\mathrm{AlPO}_{4}-5\right)$ molecular sieve-loaded novel sodium alginate composite membranes for pervaporation dehydration of aqueous-organic mixtures near their azeotropic compositions. Journal of Membrane Science 282: 473-483.

201. Adoor SG, Manjeshwar LS, Bhat SD, Aminabhavi TM (2008) Aluminumrich zeolite beta incorporated sodium alginate mixed matrix membranes for pervaporation dehydration and esterification of ethanol and acetic acid. Journal of Membrane Science 318: 233-246.

202. Patil MB, Veerapur RS, Patil SA, Madhusoodana CD, Aminabhavi TM (2007) Preparation and characterization of filled matrix membranes of sodium alginate incorporated with aluminum-containing mesoporous silica for pervaporation dehydration of alcohols. Separation and Purification Technology 54: 34-43.

203. Yeom CK, Lee KH (1998) Characterization of Sodium Alginate Membrane Crosslinked with Glutaraldehyde in Pervaporation Separation. Journal of Applied Polymer Science 67: 209-219.
204. Kim YJ, Yoon KJ, Ko SW (2000) Preparation and Properties of Alginate Superabsorbent Filament Fibers Crosslinked with Glutaraldehyde. Journal of Applied Polymer Science 78: 1797-1804.

205. Chan AW, Whitney RA, Neufeld RJ (2008) Kinetic Controlled Synthesis of pH-Responsive Network Alginate. Biomacromolecules 9(9): 25362545.

206. Chan AW, Whitney RA, Neufeld RJ (2009) Semi synthesis of a Controlled Stimuli-Responsive Alginate Hydrogel. Biomacromolecules 10(3): 609-616.

207. Abulateefeh SR, Taha MO (2014) Enhanced drug encapsulation and extended release profiles of calcium-alginate nanoparticles by using tannic acid as a bridging cross-linking agent. Journal of Microencapsulation 32(1): 96-105.

208. Sun J, Tan H (2013) Alginate-Based Biomaterials for Regenerative Medicine Applications. Materials 6: 1285-1309.
Submission Link: $\quad$ Submit Article

DOI: $10.32474 /$ DDIPIJ.2019.03.000155

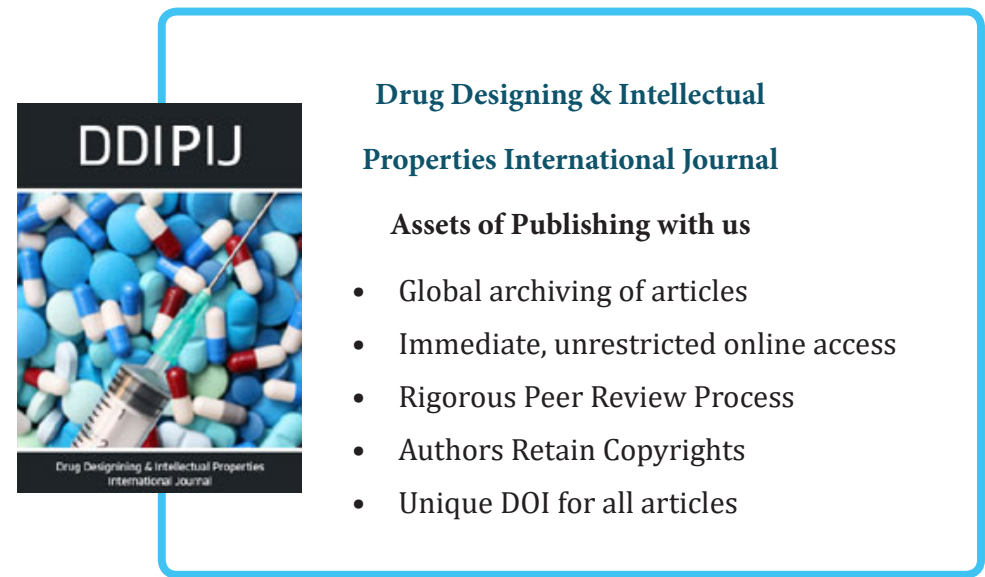

